\title{
Development and Characterization of a Novel Piezoelectric-Driven Stick-Slip Actuator with Anisotropic-Friction Surfaces
}

\author{
A Thesis Submitted to the College of Graduate Studies and Research \\ in Partial Fulfillment of the Requirements \\ for the Degree of Master of Science \\ in the Department of Mechanical Engineering \\ University of Saskatchewan \\ Saskatoon
}

By

Qingshu Zhang

December 2008

(C) Copyright Qingshu Zhang, December 2008. All rights reserved. 


\section{PERMISSION TO USE}

In presenting this thesis in partial fulfilment of the requirements for a Postgraduate degree from the University of Saskatchewan, I agree that the Libraries of this University may make it freely available for inspection. I further agree that permission for copying of this thesis in any manner, in whole or in part, for scholarly purposes may be granted by the professor or professors who supervised my thesis work or, in their absence, by the Head of the Department or the Dean of the College in which my thesis work was done. It is understood that any copying or publication or use of this thesis or parts thereof for financial gain shall not be allowed without my written permission. It is also understood that due recognition shall be given to me and to the University of Saskatchewan in any scholarly use which may be made of any material in my thesis.

Requests for permission to copy or to make other use of material in this thesis in whole or part should be addressed to:

Head of the Department of Department of Mechanical Engineering

University of Saskatchewan

Saskatoon, Saskatchewan, S7N 5A9 


\section{ABSTRACT}

Piezoelectric actuators (PEA) hold the most promise for precision positioning applications due to their capability of producing extremely small displacements down to $10 \mathrm{pm}$ $\left(1 \mathrm{pm}=10^{-12} \mathrm{~m}\right)$ as well as their high stiffness and force output. The piezoelectric-driven stickslip (PDSS) actuator, working on the friction-inertia concept, has the capacity of accomplishing an unlimited range of motion. It also holds the promises of simple configuration and low cost. On the other hand, the PDSS actuator has a relatively low efficiency and low loading capability, which greatly limits its applications. The purpose of this research is to improve the performance of the PDSS actuators by employing specially-designed working surfaces.

The working surfaces, referred as anisotropic friction (AF) surfaces in this study, can provide different friction forces depending on the direction of relative motion of the two surfaces, and are used in this research to accomplish the aforementioned purpose. To fabricate such surfaces, two nanostructure technologies are employed: hot filament chemical vapour deposition (HFCVD) and ion beam etching (IBE). The HFCVD is used to deposit diamond on silicon substrates; and the IBE is used to etch the diamond crystalloid with a certain angle with respect to the coating surface to obtain an unsymmetrical-triangle microstructure. 
A PDSS actuator prototype containing the AF surfaces was developed in this study to verify the function of the AF surfaces and characterize the performance of PDSS actuators. The designed surfaces were mounted on the prototype; and the improvement in performance was characterized by conducting a set of experiments with both the normal isotropic friction (IF) surfaces and the AF surfaces, respectively. The results illustrate that the PDSS actuator with the AF surface has a higher efficiency and improved loading capability compared to the one with the IF surfaces.

A model was also developed to represent the displacement of the novel PDSS actuator. The dynamics of the PEA and the platform were approximated by using a second order dynamic system. The pre-sliding friction behaviour involved was investigated by modifying the LuGre friction model, in which six parameters (Note that three parameters are used in the LuGre model) were employed to represent the anisotropic friction. By combining the PEA mechanism model, the modified friction model, and the dynamics of end-effector, a model for the PDSS actuator with the AF surface was developed. The model with the identified parameters was simulated in MATLAB Simulink and the simulation results obtained were compared to the experimental results to verify the model. The comparison suggests that the model developed in this study is promising to represent the displacement of the novel PDSS actuators with AF surfaces. 


\section{ACKNOWLEDGMENTS}

I would like to express my appreciation to those people who have contributed to this research work.

At First, I would like to give my sincere gratitude to my supervisors, Professors Daniel Chen and Chris Zhang, for their support and the guidance to this research work. Their priceless suggestion and encouragement not only drive me to fulfill present research project but will also benefit me for my further career.

I also deliver my sincere appreciation to Professor Yang Shi and Professor Richard Burton for being my advisory committee members. My earnest gratitude also goes to Professor Qiaoqin Yang, Professor Akira Hirose, as well as Dong Kang, Yongji Tang, and Wenwen Yi for their precious help and thoughtful consideration during my study. Besides, my appreciation goes to Professor David Summer, Professor James Bugg and Professor Walerian Szyszkowski, Ms. Kelley Neale, Mr. Doug Bitner and Mr. Hans Steinmetz who have helped and supported me a lot since the beginning of the research.

Another truthfully acknowledgment goes to the Department of Mechanical Engineering for its financial support though a teaching fellowship.

Thank all those people who gave me the best memory in the University of Saskatchewan. 


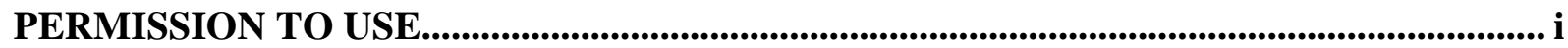

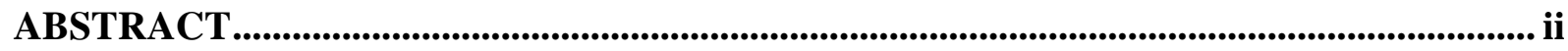

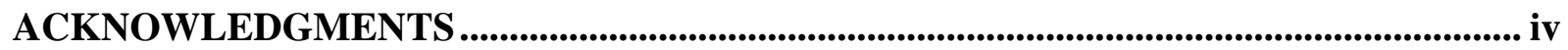

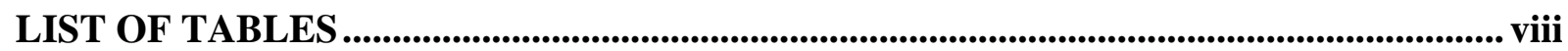

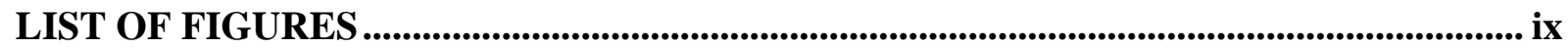

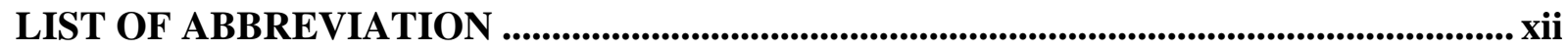

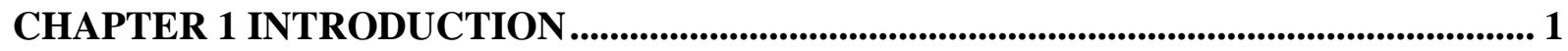

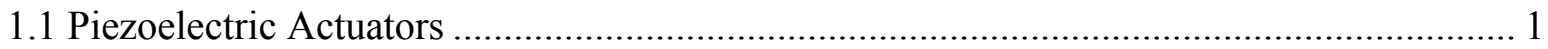

1.2 Piezoelectric-Driven Stick-Slip Actuators.................................................................... 5

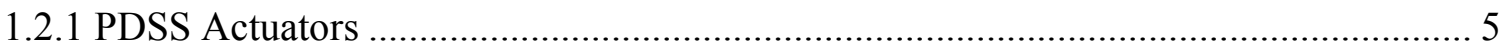

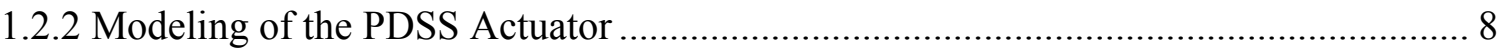

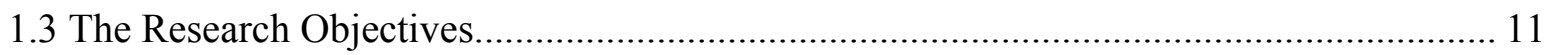

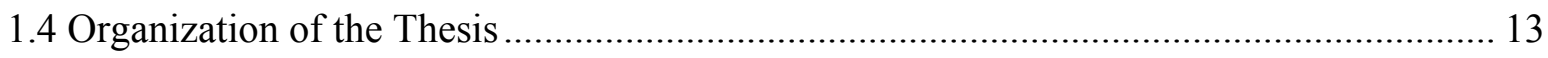

CHAPTER 2 FABRICATION OF ANISOTROPIC FRICTION SURFACES.................... 15

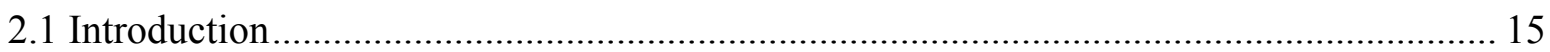




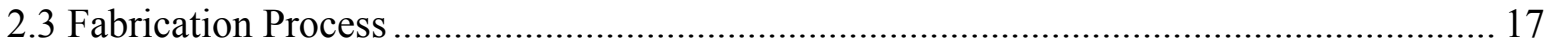

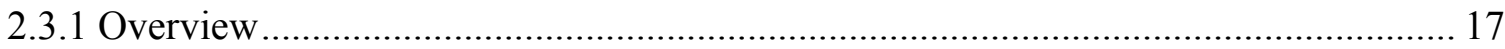

2.3.2 Hot Filament Chemical Vapor Deposition ............................................................ 19

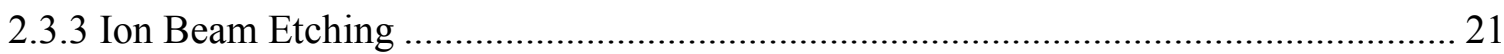

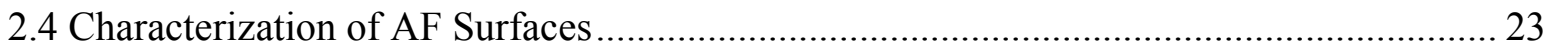

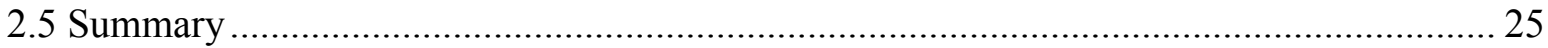

CHAPTER 3 PROTOTYPE OF A NOVEL PDSS ACTUATOR WITH AF SURFACES. 27

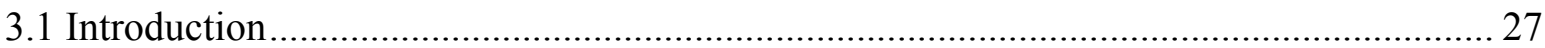

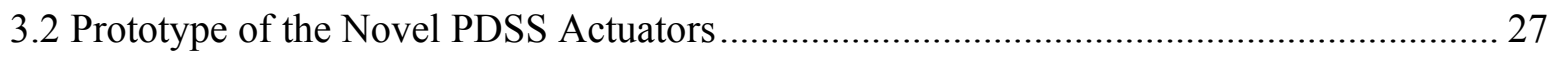

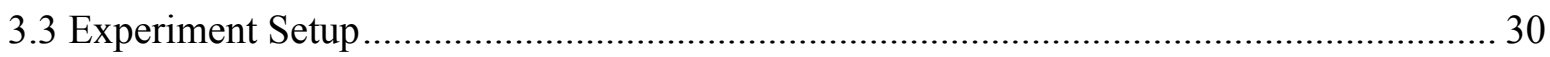

3.4 Characterization of the PDSS Actuator Performance ...................................................... 32

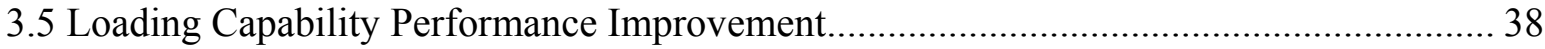

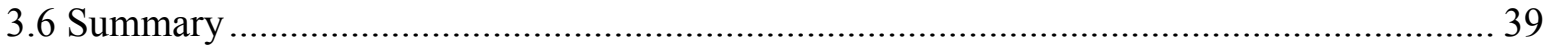

CHAPTER 4 MODELING OF THE PEA DYNAMICS .................................................... 40

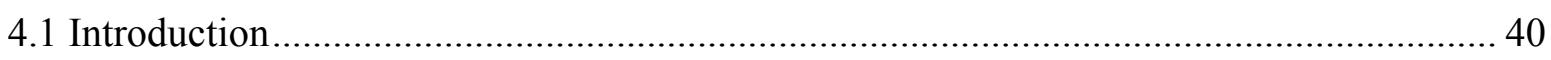

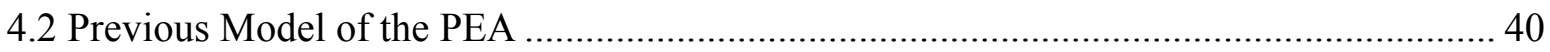

4.3 Modeling the Dynamics of Piezo-Actuator Positioning Systems ...................................... 42

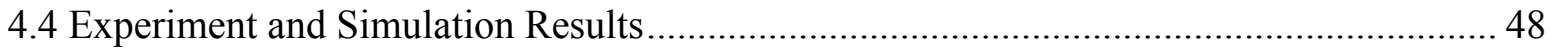

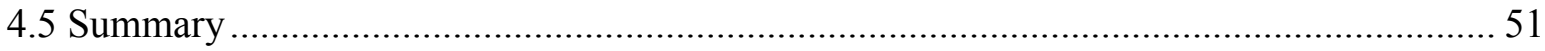


5.1 Introduction 52

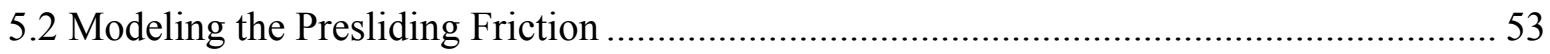

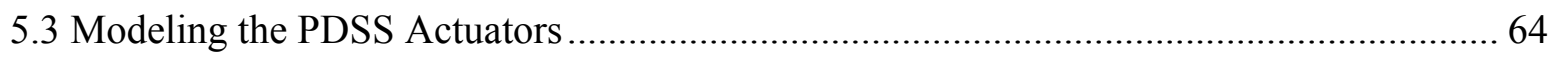

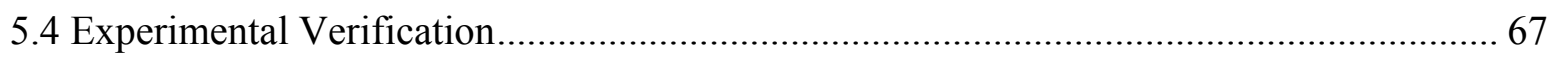

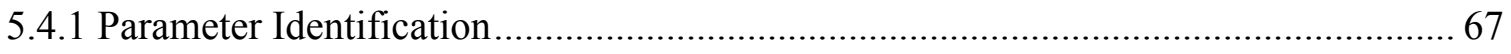

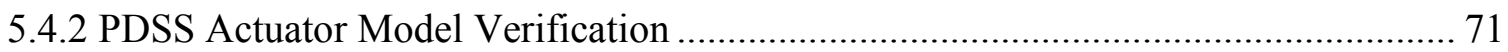

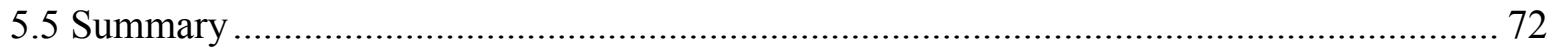

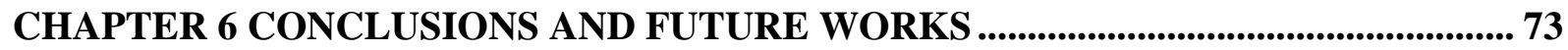

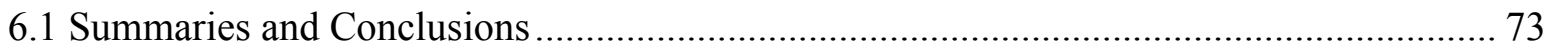

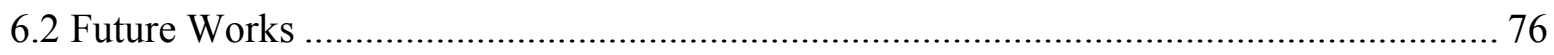

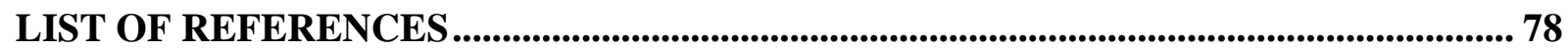

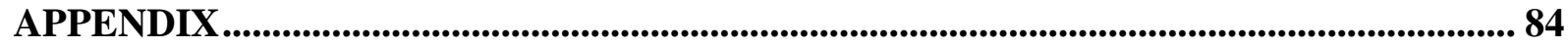

Appendix 1 MATLAB Program Used to Identify the Parameters of the Second Order System.

Appendix 2 MATLAB Program Used to Draw Figure 4.7 .............................................. 86

Appendix 3 MATLAB Program Used to Identify the Parameters in Friction Model .............. 87 


\section{LIST OF TABLES}

Table

Page

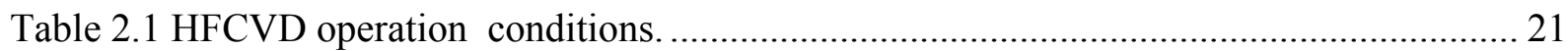

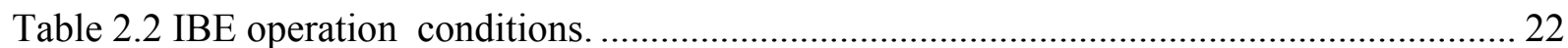

Table 3.1 Efficiency improvement using AF surfaces............................................................. 37 


\section{LIST OF FIGURES}

Figure $\quad$ Page

Figure 1.1 Schematic of the PEA stack [Niezrecki et al., 2001]. ............................................. 3

Figure 1.2 Cymbal actuator in original and deformed states [Dogan et al., 1996] ....................... 4

Figure 1.3 Frequency-leveraged inchworm actuator [Niezrecki et al., 2001]...........................5

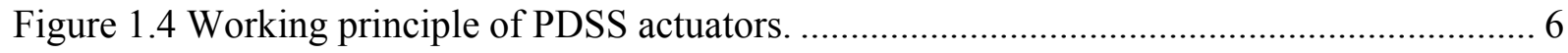

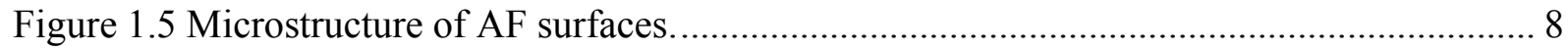

Figure 1.6 Model of a PDSS actuator by Chang and Li [Chang and Li, 1999].......................... 10

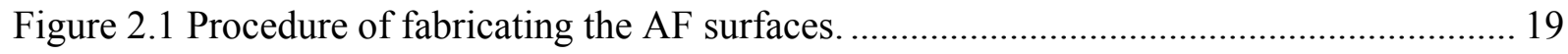

Figure 2.2 (a) HFCVD equipment and (b) Overview of HFCVD principle............................... 20

Figure 2.3 (a) IBE equipment and (b) Overview of working principle of IBE equipment........... 22

Figure 2.4 Representative SEM micrographs (top-view) of (a) the deposited diamond coating and

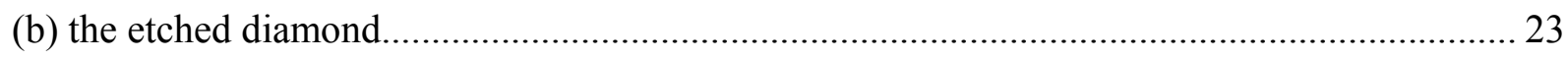

Figure 2.5 Representative SEM micrographs of (a) the deposited diamond coating and (b) the

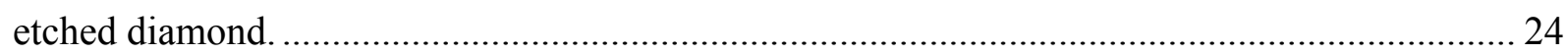

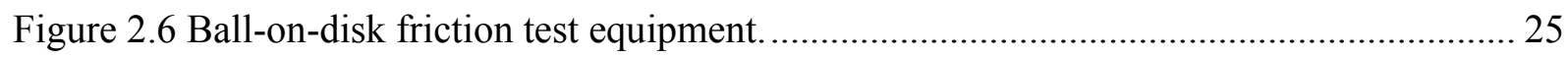

Figure 2.7 Friction coefficients in the ball-on-disk tests. ..................................................... 25 
Figure 3.1 Schematic configuration of PDSS actuator prototype

Figure 3.2 Photo of the PDSS actuator prototype.

Figure 3.3 Silicon plates glued on end-effector and platform.

Figure 3.4 The test rig.

Figure 3.5 Virtual control panel of the ControlDesk in dSPACE.

Figure 3.6 Voltage signals used in driving the PDSS actuator: (a) forward and (b) backward.... 33

Figure 3.7 Comparison of the measured displacements of the end-effector driven by the PDSS

actuator with IF surfaces.

Figure 3.8 Comparison of the measured displacements of the end-effector driven by the PDSS

actuator with AF surfaces.

Figure 3.9 Experimental result of performance of PDSSA using two surfaces... 36

Figure 3.10 Setup of the force sensor.

Figure 3.11 Measured maximum output force of PDSS actuators with IF surfaces and AF surfaces under different inputs.

Figure 4.1 (a) Total system of PEA and the mass-stage; (b) Force analysis of the system of a PEA and a mass-stage [Adriaens et al., 2000].

Figure 4.2 Piezo-actuated positioning systems.

Figure 4.3 Block diagram representing the displacement of the positioning mechanism. 46

Figure 4.4 Graphical representation of $\left|\emptyset_{2}(\mathrm{~L}) / \emptyset_{1}(\mathrm{~L})\right|$ 47

Figure 4.5 Piezo-actuated positioning system. 48

Figure 4.6 Measured and simulated step responses for a mass ratio of 138.8 . 50 
Figure 4.7 Errors involved in the second-order approximations for an applied voltage of $48.2 \mathrm{~V}$.

Figure 5.1 Friction behaviors: (a) The relation between friction and displacement as found by Robinowicz (b) shows how the friction force may decrease contimuously from thestatic friction

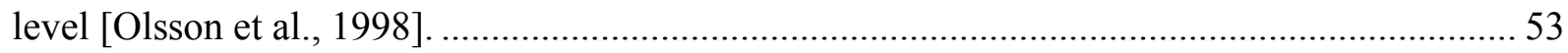

Figure 5.2 Realization of the direction-dependent parameter in Simulink............................... 61

Figure 5.3 Simulink model based on the Simplified LuGre model with 3 parameters. …........... 62

Figure 5.4 Simulink model based on the modified LuGre model with 6 parameters.................. 63

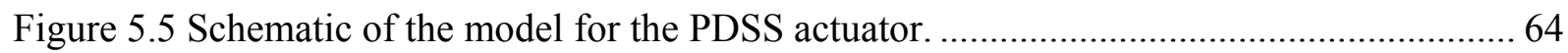

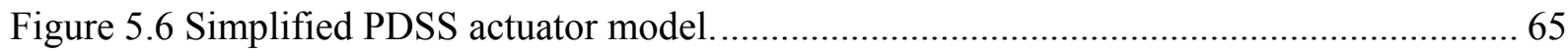

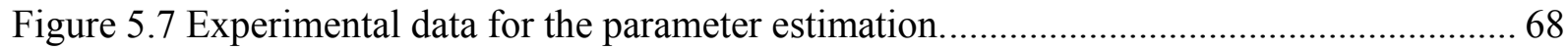

Figure 5.8 Simulink model used to simulate the motion of end-effector in two directions. ........ 69

Figure 5.9 Comparison of the measured end-effector displacement and model predictions........ 70

Figure 5.10 Experimental result matches simulation result.................................................. 70

Figure 5.11 Verification of the novel PDSS actuator model through the comparison between experimental result and simulation result under $64 \mathrm{~V} / 20 \mathrm{~Hz}$ inputs.......................................... 71 


\title{
LIST OF ABBREVIATION
}

\author{
Abbreviation: \\ $A$ \\ cross-sectional area of the PEA, $m^{2}$ \\ $A F$ \\ anisotropic friction \\ $C$ \\ steady state gain \\ $c$ \\ damping ratio of the combination of the PEA and the stage, $\mathrm{kg} / \mathrm{s}$ \\ $c_{g}$ \\ damping coefficient of PEA and mass-stage, $\mathrm{kg} / \mathrm{s}$ \\ $c_{S}$ \\ stage damping coefficient, $\mathrm{kg} / \mathrm{s}$ \\ CVD \\ chemical vapour deposition \\ $D_{v f}$ \\ displacement to voltage proportion of piezoelectric actuator, $\mathrm{mm} / \mathrm{V}$ \\ $d y$ \\ an element of infinitesimal thickness of PEA \\ E \\ Young's modulus \\ $E I$ \\ error index \\ $E_{v f}$ \\ force to voltage proportion of for piezoelectric actuator, $N / V$ \\ $F_{C}$ \\ Coulomb friction, $N$ \\ $F_{b}$ \\ break-away friction, $N$ \\ $F_{f}$ \\ friction force, $N$ \\ $F_{p}$ \\ force generated by the piezoelectric actuator, $N$ \\ $F_{S}$ \\ interaction force between the PEA and the stage, $N$
}


$F_{S S}$

$G(s)$

$g(\dot{x})$

HFCVD

$I B E$

IF

$j$

$k$

$k_{g}$

$k_{S}$

$L$

$M$

$M D$

$m$

$m_{e}$

$m_{g}$

$m_{s}$

$N(y, t)$

$M W C V D$

PEA

PDSSA

PZT

$q_{j}(t)$ a force simplified from $g(\dot{x})$ function, $N$

transfer function of the PEA and the stage system

a function to describe the Stribeck effect

hot filament chemical vapour deposition

ion beam etching

isotropic friction

number of mode

stiffness of the combination of the PEA and the stage, $\mathrm{kg} / \mathrm{s}^{2}$

stiffness of PEA and mass-stage, $\mathrm{kg} / \mathrm{s}^{2}$

mass-stage stiffness, $\mathrm{kg} / \mathrm{s}^{2}$

full length of the PEA, $\mu m$

mass of the mass-stage, $\mathrm{kg}$

measured displacement, $\mathrm{mm}$

mass of the PEA, $k g$

mass of the end-effector, $\mathrm{kg}$

mass of the PEA and the mass-stage, $\mathrm{kg}$

stage mass, $\mathrm{kg}$

forces on the side of the element

microwave chemical vapour deposition

piezoelectric actuator

piezoelectric-driven stick-slip actuator

piezoelectric ceramics

mode coordinate 
coefficient of determination

forward displacement of end-effector in one step, $\mathrm{mm}$

simulated displacement, $\mathrm{mm}$

backward displacement of end-effector in one step, $\mathrm{mm}$

sign of $z$

sign of the velocity

electrical-mechanical transformer ratio, $N / V$

time, $s$

voltage input in frequency domain

voltage applied to PEA, $V$

voltage signal applied to the PEA, $V$

Stribeck velocity, $\mathrm{mm} / \mathrm{s}$

a distance of element has moved from its equilibrium position

plastic (irreversible) displacement of friction, $\mathrm{mm}$

velocity of the plastic deformation, $\mathrm{mm} / \mathrm{s}$

displacement of friction, $\mathrm{mm}$

velocity of a sliding object, $\mathrm{mm} / \mathrm{s}$

displacement of the end-effecter, $\mathrm{mm}$

displacement output of the PEA positioning system in frequency domain displacement of the PEA positioning system, $\mathrm{mm}$

position of PEA or that of its one element

elastic (reversible) displacement of friction, $\mathrm{mm}$

velocity of the elastic deformation, $\mathrm{mm} / \mathrm{s}$ 
breakaway displacement, $\mathrm{mm}$

steady-state elastic displacement, $\mathrm{mm}$

parameter of the stress-strain relation determination

piecewise continuous function of the Elastoplastic friction model

normal mode function

voltage difference applied to the piezoelectric actuator, $V$

expanding displacement of piezoelectric actuator, $\mathrm{mm}$

slide of end-effector on platform in one step, $\mathrm{mm}$

strain

damping ratio

viscous coefficient of the PEA

step efficiency

mass density of the PEA, $\mathrm{kg} / \mathrm{m}^{3}$

stiffness coefficient

stress

coefficient of the contact stiffness, $N / \mathrm{mm}$

coefficient of the contact stiffness in direction $\mathrm{A}, \mathrm{N} / \mathrm{mm}$

coefficient of the contact stiffness in direction $\mathrm{B}, \mathrm{N} / \mathrm{mm}$

damping coefficient of the tangential compliance, $\mathrm{Ns} / \mathrm{mm}$

damping coefficient of the tangential compliance in direction $\mathrm{A}, \mathrm{Ns} / \mathrm{mm}$ damping coefficient of the tangential compliance in direction $\mathrm{B}, \mathrm{Ns} / \mathrm{mm}$ parameter of the viscous friction, $\mathrm{Ns} / \mathrm{mm}$

natural frequency, $\mathrm{rad} / \mathrm{s}$ 


\section{CHAPTER 1 INTRODUCTION}

\subsection{Piezoelectric Actuators}

An actuator is generally a transducer for activating or controlling a mechanism or system. The input to an actuator is electric energy, pneumatic energy, hydraulic energy, or thermal energy; and the output is usually mechanical energy such as motion or moment. A piezoelectric actuator (PEA) is a type of electric actuators utilizing electric energy based on piezoelectric effect. PEAs are made of special dielectric materials called piezoelectric materials and these materials have the following features:

1. An externally applied force on them induces electrical charge;

2. Applied electrical charge on them induces force which causes its elongation as well.

The first feature is referred as the piezoelectric effect; and the second one as the converse piezoelectric effect. The piezoelectric effects build the connection between the electrical and mechanical domain, enabling piezoelectric materials suitable to be used as transducers. The piezoelectric effect can be used for building sensors; while the converse piezoelectric effect is used for building actuators.

The piezoelectric effect was first discovered in the natural crystals, such as Rochelle salt and quarts, by Curic brothers in 1880s. In natural piezoelectric materials, the free strains induced 
by piezoelectric effect are in an order of $0.1 \%$ to $0.2 \%$ of the material size [Park and Shrout, 1997]. The piezoelectric effect had not been applied to any practical use for a long time until man-made piezoelectric ceramics was successfully produced. The man-made piezoelectric ceramics (PZT) have more significant piezoelectric effects than natural materials do. As a result, a lot of actuators and sensors were developed based on them [Adriaens et al., 2000]. Although the man-made piezoelectric materials have a bigger piezoelectric effect, the effect is still in a tiny level of 1\% [Park and Shrout, 1997]. The feature of small effect level gives the PEA high precision; but on the other side, it also restricts the long range of displacement output from the PEA. To increase the displacement range of the PEA, a number of methods have been developed in the literature. Basically, these methods can be divided into three categories, leading to the development of three types of actuators: namely internally leveraged actuators, externally leveraged actuators, and frequency leveraged actuators [Niezrecki et al., 2001].

The internally leveraged actuator uses the internal structure to generate amplified displacement without the use of external mechanical components. The external leveraged actuator uses the external mechanical component such as a lever to amplify the displacement of the PEA. The frequency leveraged actuators are based on the alternating input signals to generate repeatable motions. These three methods can be either used independently or combined together, for example, the use of an internal leveraged actuator as a component of external leveraged actuators. Some typical examples of the aforementioned actuators are introduced in the following.

The stacked PEA is a typical internally leveraged actuator. The schematic of a PEA stack 
is shown in Figure1.1. In the PEA stack, a number of piezoelectric patch layers are connected in series, and between every two layers is the conductive epoxy connected to the external electrodes. When the voltage is applied on the external electrode, each small wafer is activated; and the deformation generated is accumulated to form the overall elongation of the stack. It is noted that the range of output displacement of stacked PEA is still limited. Thus, it is widely used as the input to the externally leveraged actuators or frequency leveraged actuators.

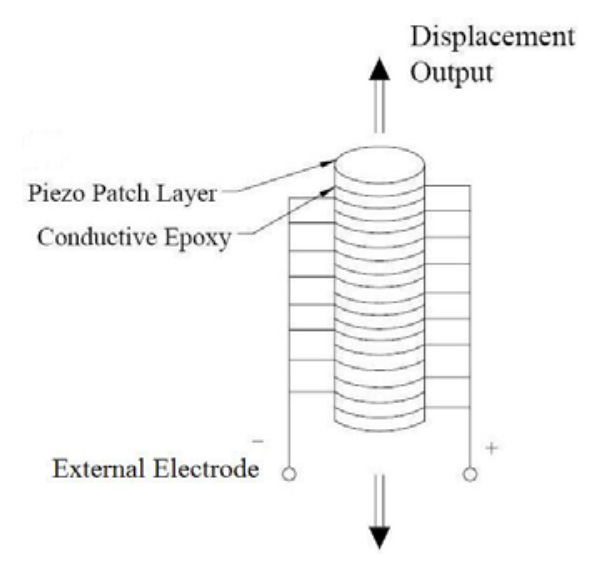

Figure 1.1 Schematic of the PEA stack [Niezrecki et al., 2001].

Figure 1.2 shows a Cybal actuator, an example of externally leveraged actuators. This actuator uses a simple lever arm to amplify the displacement of PEA at the cost of sacrificing the output force. This method can amplify the displacement by 10 times. 


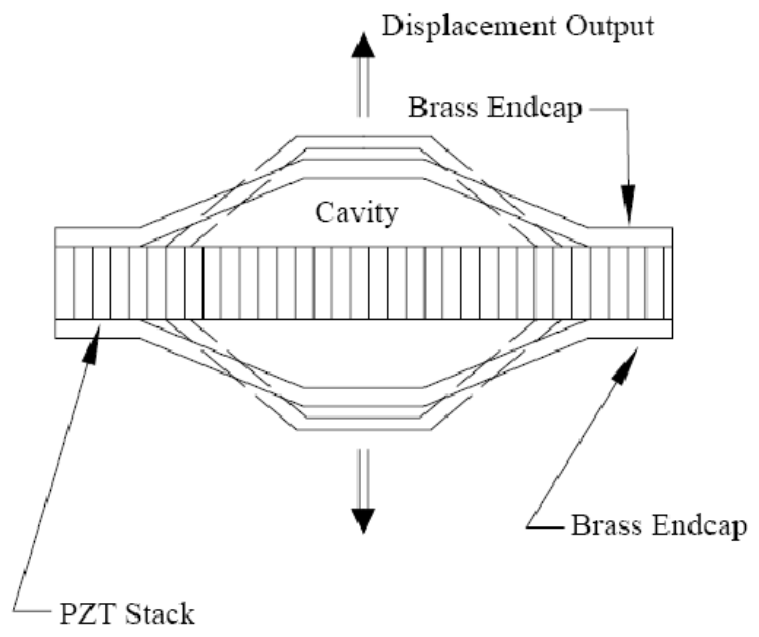

Figure 1.2 Cymbal actuator in original and deformed states [Dogan et al., 1996].

The Inchworm, which was developed and patented in 1970s [Burleigh, 1998], is a typical frequency leveraged actuator and is able to achieve unlimited displacement by rapidly moving the actuators in one direction in a series of small steps. Its structure is shown schematically in Figure 1.3. The Inchworm usually consists of three PEAs to work in sequence, which are the leading actuator, trailing actuator, and extending actuator. In the first step the leading PEA clamps the rod and the trailing PEA unclamps; in the second step, the extending PEA extends to the right and so does the leading PEA; and in the last step, the leading PEA unclamps rod, the trailing PEA clamps, and the PEA contracts, which brings the leading PEA back to initial position but the rod stays. As a result, the rod is moved forward a distance equals to the PEA extension. By repeating these steps, a theoretically unlimited displacement can be achieved. It is seen that an inchworm actuator needs three PEAs causing the complexity in architecture and thus the relatively high cost. The merits of the inchworm are its high output force, well defined step size, and significantly long range of motion. 


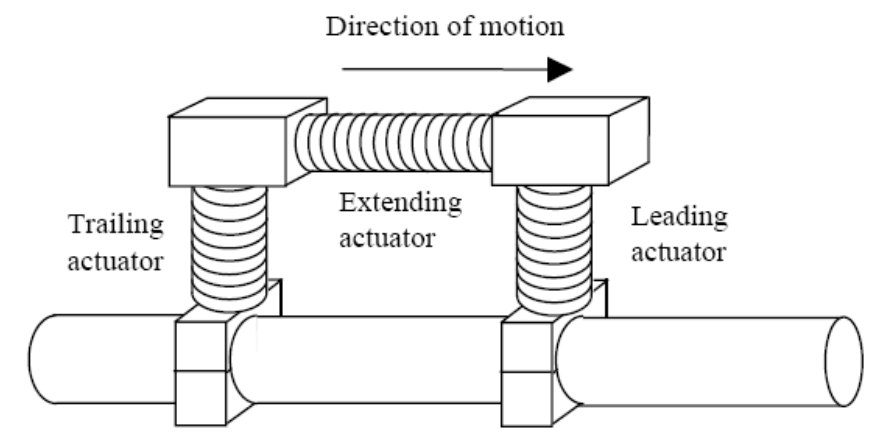

Figure 1.3 Frequency-leveraged inchworm actuator [Niezrecki et al., 2001].

Piezoelectric-Driven Stick-Slip actuator (PDSS actuator) is also used to accomplish the unlimited range of motion, which is introduced in the following section.

\subsection{Piezoelectric-Driven Stick-Slip Actuators}

\subsubsection{PDSS Actuators}

The working principle of PDSS actuators is based on the friction-inertia concept. This concept is shown schematically in Figure 1.4, in which Object 1 is a PEA, Object 2 is a movable platform driven by the PEA, and Object 3 is an end-effector supported and guided by the platform. In Figure 1.4 (a), three objects are all in their initial positions. Under the action of the PEA expansion in Figure 1.4 (b), both the platform and the end-effector move to the right with a distance denoted by $S$; and under the action of the PEA contraction in Figure 1.4 (c), the platform moves back to its initial position and the end-effector also attempts to move back. However, if the acceleration due to the contraction is large enough, the force on the end-effector due to its inertia becomes greater than the friction between the contact surfaces or working surfaces of the 
platform and end-effector. As a result, the end-effector will slide on the platform, resulting in a motion denoted by $\Delta S$ in Figure 1.4 with respect to its original position. By repeating the aforementioned PEA expansion and contraction, the end-effector will move "continuously" and reach a theoretically-unlimited displacement. However, it should be noted that the output force depends on the friction between the end-effector and movable platform. Even though as the PDSS actuators have still found their wide applications, where the critical issue is not output force but the displacement capability, such as in the fields of scanning microscopy, micro robotics, and Microsystems [Bergander et al., 2003; Pohl, 1987; Renner et al., 1990; Chang and Li, 1999; Hoogeman et al., 1998; Zhang et al., 2006]. Compared to the inchworm and amplifying leveraged actuators, the PDSS actuators hold the promises of simple configuration, low cost, etc. [Bergander et al., 2003; Chang and Li, 1999]

(a) Start

(b) Expansion

(c) Contraction and end

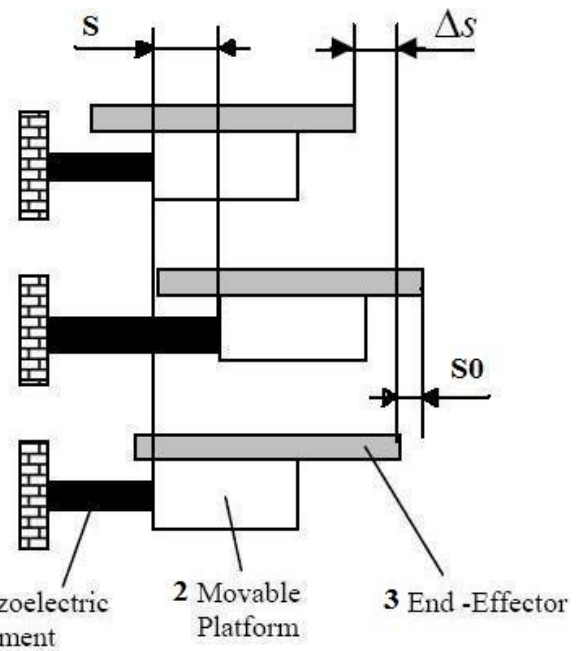

Figure 1.4 Working principle of PDSS actuators.

For such a PDSS actuator, the friction between the working surfaces of the platform and end-effector is critically important. Ideally, the friction is infinite during the course of the PEA expansion such that the end-effector can stick with the platform, whereas it is zero during the 
course of the PEA contraction such that the end-effector can slip or slide on the platform. In reality, however, the working surfaces of existing PDSS actuators were all engineered by means of traditional manufacturing methods, resulting in the isotropic friction [Chang and $\mathrm{Li}, 1999$ ].

It is noticed that, although the end-effector does not go to the initial position completely at the end of each working cycle, it goes back a little distance, denoted by $S_{0}$. For a given $S$, the smaller the $S_{0}$ is, the larger the $\Delta S$ is. In one working cycle, the larger $\Delta S$ means higher efficiency, so deceasing $S_{0}$ provides a means to enhance the efficiency of the actuator. In existing PDSS actuators, $S_{0}$ is relatively large due to the limited friction between the platform and the end-effector, causing low efficiency. Also, due to the limited friction, existing PDSS actuators have the low loading capability friction. If a large loading force is applied on the end-effector, the friction force activating the end-effector may not be large enough to overcome the loading force. As a result, the end-effector cannot move forward and thus the PDSS actuator does not work in this case.

In summary, existing PDSS actuators have two defects: relatively low efficiency and low loading capability. These two defects greatly limit the applications of the PDSS actuators. The aim of this research is to develop methods to enhance the performance of the PDSS actuators with improved efficiency and loading capability.

In this research, the anisotropic-friction (AF) surfaces are proposed to be used for the working surfaces of the PDSS actuators, i.e., the contact surfaces of the end-effector and the platform. Figure 1.5 shows the proposed microstructure of these AF surfaces in which the angle 
$\alpha$ is larger than angle $\beta$. Obviously, the surfaces with a larger $\alpha$ can provide a larger static friction when Object 2 moves to left; and the surfaces with a smaller $\beta$ can provide a less static friction when the Object 2 moves to right. That means Object 3 is easier to slide to the left than to the right relative to Object 2 . This easier-sliding property can bring a smaller $S_{0}$ and thus a larger $\Delta S$. As a result, manipulating these two angles of the microstructure provides a method to control $S_{0}$ and $\Delta S$, thereby improving the efficiency of the PDSS actuators. Also, since a larger $\alpha$ provides the larger static friction when Object 2 moves to left, increasing in $\alpha$ can improve the loading capability of the PDSS actuators. A recent research [Yang et al, 2008] shows that the size and the shape of the nanotips formed on a substrate by means of nano-technologies could be controlled by appropriately manipulating the operating conditions. This suggests that it is possible to achieve the proposed AF surfaces, as applied to the improvement of the PDSS actuators.

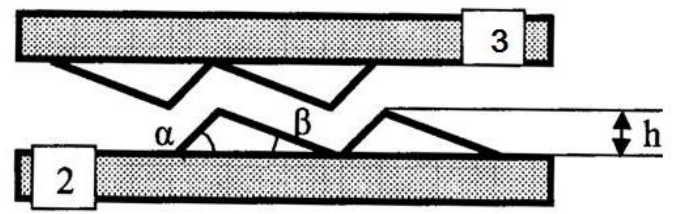

Figure 1.5 Microstructure of AF surfaces.

\subsubsection{Modeling of the PDSS Actuator}

Although PDSS actuators have drawn extensive attention for applications, research in this area is still in its early stage and many issues remain to be addressed. One key issue is the representation of the dynamic displacement of the end-effector. It is known that two factors can 
significantly contribute to the dynamics of the end-effector displacement, which are the dynamics of piezoelectric element and the friction behaviour involved in PDSS actuator. For the first factor, the dynamics of piezoelectric element is usually modeled approximately by a secondorder system empirically when the piezoelectric element is used to drive a positioning mechanism [Adriaens et al., 2000]. However, the rationale for such an approximation is lacking in the literature, thus leaving some issues unaddressed, such as the scope of and the error associated with approximation. For the second factor, the friction behaviour involved in PDSS actuator has been represented by using friction models such as Coulumb friction model, Dahl friction model, et al. [Chang and Li, 1999; Darby and Pellegrino, 1999; Chen et al., 2008]. It is noted that these models were developed for traditional isotropic-friction (IF) surfaces. These models were adopted and used to represent the friction on the IF surfaces in previous studies.

In the Pohl's study [Pohl, 1987], the PZT was treated as a linear component, thus the relationship between the PEA motion and the driving voltage was represented by a linear function. Specifically, the displacement and the force generated by PZT are related to the voltage applied to the PEA by, respectively

$$
\begin{aligned}
\Delta X & =D_{v f} \times \Delta V \\
F_{p} & =E_{v f} \times \Delta V
\end{aligned}
$$

where $\Delta X$ is the expanding displacement of piezoelectric actuator, $F_{p}$ is the force generated by the piezoelectric actuator's expansion; $\Delta V$ is the voltage applied to the piezoelectric actuator; and 
$E_{v f}$ and $D_{v f}$ are coefficients, relating the voltage to the force and the displacement, respectively. It is noted that this model does not include the PEA dynamics.

Another study of modeling the PDSS actuator was reported by Chang and Li [Chang and $\mathrm{Li}$, 1999]. In this work, the friction between the end-effector and movable platform is represented by the Coulomb friction model, and the dynamics of the piezoelectric actuator is modeled as a mass-spring-damping system shown schematically in Figure 1.6. Based on this model, Chang and Li also carried out simulations to predict the actuator's performance. It was noticed that the use of the Coulomb friction model to represent the friction behaviour in such a stick-slip actuator may not be appropriate. Since in a small motion such as several microns, the friction is dominated by the presliding displacement, i.e., the motion prior to fully developed slip, and its nature is inherently different from the Coulomb friction [Dupont, 2002; Ferretti, 2004].

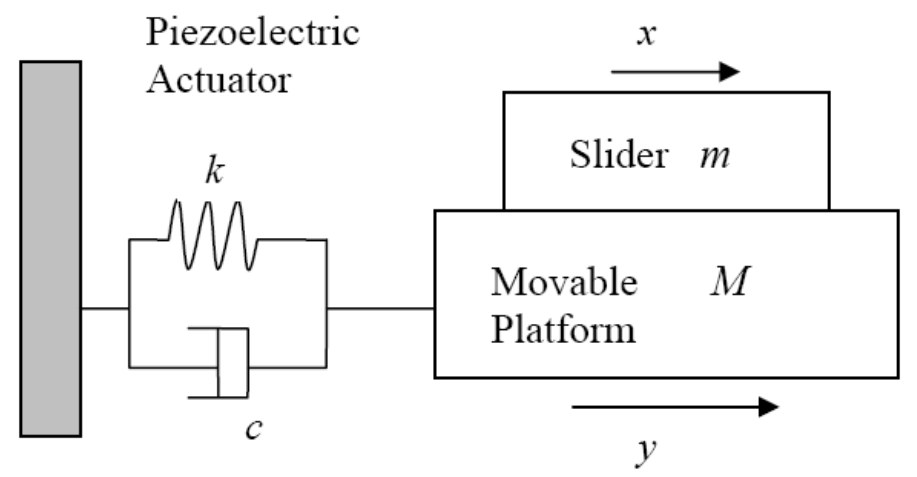

Figure 1.6 Model of a PDSS actuator by Chang and Li [Chang and Li, 1999].

A recent study in this area was reported by the Kang and Chen [Chen and Kang, 2008] on modeling of PDSS actuator with IF surfaces. In their study, the PEA was modeled as a second order system and the friction involved was represented by the simplified LuGre model. The 
simulations were conducted in Simulink and the results obtained were compared to the experimental results to validate the developed model.

Although some PDSS actuator models have been successfully developed to represent the displacement of the end-effector, none of existing models can represent the effect of AF surfaces which provides friction depending on the relative motion between end-effector and platform. Thus, there is a need to adopt and extend existing model for representing the friction behaviour of the AF surfaces.

\subsection{The Research Objectives}

The research is aimed at developing methods to enhance the performance of the PDSS actuators and developing models to represent the enhanced performance. Specifically, the following objectives are set to be achieved:

\section{(1) Use the nano-technologies to construct the surfaces so as to achieve the anisotropic friction}

As illustrated in Figure 1.4, the contact surfaces or working surfaces of end-effector and platform are critical to the performance of the piezoelectric-driven stick-slip actuator. The working surfaces should be engineered such that, ideally, the friction is infinite during the stick phase, whereas it is zero during the sliding phase. In other words, the working surfaces should be engineered in order that the friction between them depends on the direction of their relative 
motion. For this purpose, the nano-technologies will be employed to construct the working surfaces so as to achieve the desired friction behavior.

(2) Prototype novel PDSS actuators with the anisotropic-friction surfaces and characterize the actuator performance.

A prototype of the novel PDSS actuator will be designed and built, in which the AF surfaces will be mounted on the contact surfaces of the platform and the end-effector forming the working surfaces. Experiments will be conducted to characterize the performance of the novel PDSS actuator in terms of the efficiency and the loading capability, as well as the relationship between the displacement and the applied voltage.

(3) Develop a model to represent the displacement of the end-effector of the novel PDSS actuator.

The development of the model involves the representation of the PEA dynamics and the representation of the friction involved and then integrates them to represent the motion of the end-effector. In this research, appropriate models will be adopted from the reported ones and integrated for the displacement representation; and experiments will be conducted to identify the parameters of the models. Once all the parameters are identified, the stick-slip actuator model will be verified experimentally. 


\subsection{Organization of the Thesis}

This thesis consists of 6 chapters. Besides this chapter, the rest 5 chapters are organized as follows:

Chapter 2: Fabrication of Anisotropic Friction Surfaces

This chapter presents the design and fabrication of the AF surfaces. In particular, the material of surfaces is selected, and two technologies of hot filament chemical vapor deposition and ion beam etching are applied to fabricate the AF surfaces. After successful fabrication, the preliminary tribological tests on the surfaces are conducted to characterize the properties of the AF surfaces.

Chapter 3 Prototype of a Novel PDSS Actuator with AF Surfaces

This chapter presents the development of a prototype of a novel PDSS actuator with AF surfaces and the characterization of its performance. The performance of the novel actuator is compared to that of a PDSS actuator with IF surfaces, showing the improvement the efficiency and loading capability of the novel PDSS actuator.

Chapter 4 Modeling of the PEA Dynamics

If a PEA is used to drive a positioning mechanism, the combined dynamics is approximated empirically by that of a second-order system. However, the rationale for such an approximation is lacking in the literature, thus leaving some issues unaddressed, such as the scope of and the error associated with the approximation. This chapter presents such a rationale and its experimental verification. 
Chapter 5 Modeling of the Novel PDSS Actuators

By modifying an existing friction model, and combining it with the PEA model, a model is developed to represent the displacement of the end-effector of the novel PDSS actuator containing the AF surfaces in this chapter. This is followed by the presentation of the experiments conducted to identify the model parameters and to verify the model.

Chapter 6 Conclusions and Future Works

This chapter presents the conclusions to this research, following by the possible future work. 


\section{CHAPTER 2 FABRICATION OF ANISOTROPIC FRICTION SURFACES}

\subsection{Introduction}

In a PDSS actuator, the friction between the contact surfaces is the force to activate the end-effector. Thus, the design and fabrication of the anisotropic friction surfaces are crucial in this research. This chapter presents the design and fabrication of the AF surfaces and the characterization tests on these surfaces. The material selection for the AF surfaces is presented in Section 2.2; and the fabrication process by employing chemical vapour deposition and ion beam etching techniques is introduced in Section 2.3. This is followed by the tests conducted on the surfaces to characterize the surface features in Section 2.4.

\subsection{Material Selection}

To select the material for the fabrication of the AF surfaces, two main factors are considered in this study; one is the requirement imposed on the AF surfaces and the other one is the fabrication process. In a PDSS actuator, the friction is the working force to drive the endeffector, the friction between the end-effector and platform is therefore crucial. If the friction produced is stable, the PDSS actuator can work stably; otherwise, the PDSS actuator shows varied performance. However, the wear between contact surfaces is inevitable, which implies the 
friction is changing as the actuator keeps on running. To ensure the PDSS actuator works stably, the effect of wear must be minimized. This suggests the contact surfaces should have excellent wear-resistance.

As mentioned previously, the AF surfaces should have the microstructure shown in Figure 1.5. The size of the triangle tips in the microstructure is another design issue. Since the PEA is supposed to elongate/contract in a very small range (typically several microns), the microstructure would make no sense if the tips are too big. Ideally, the tips should be in the size of nanometers, which is much smaller than the PEA elongate/contract motion. In order to obtain this type of nanotips on the surfaces, nano-technologies are considered to deposit small crystalloids onto a substrate. For this, another requirement imposed on the materials is that they must have good adhesion with the substrate. Based on the above discussion, in summary the requirements imposed on the materials for the AF surfaces must have high hardness, low wear rate, and strong adhesion to the substrates.

It is known that diamond is the hardest material in nature and the wear rate when diamond surfaces slide over each other is extremely low. The state-of-the-art-of chemical vapor deposition (CVD) techniques have enabled the successful synthesis of diamond crystals and thin films on various substrates. Adherent diamond thin films can be grown on silicon wafers with hydrogen and methane gas mixture at low temperature $\left(\sim 1000^{\circ} \mathrm{C}\right)$ and low pressure (vacuum). So-obtained diamond has mechanical and tribological properties comparable to those of natural diamond. Besides the diamond, Boron-Nitride is also a material processing similar hardness with diamond. And it can also be coated by using CVD like diamond coating. Although Boron-Nitride 
thin film has high hardness and is able to be coated, the synthesis technique for it is not as mature as diamond coating, and the substrate holding good adhere for its CVD is not as common as silicon (the substrate for diamond).

The superior properties and mature synthesis technique make CVD diamond coating on silicon substrate most suitable for the AF surfaces fabrication. Finally the diamond is chosen as the surface material for the following reasons: it has high hardness which means excellent wearresistance; it has good adhesion with silicon; the size of diamond crystalloid can be controlled in the micron level by choosing the deposition parameters appropriately [Yang and Tang, 2008].

\subsection{Fabrication Process}

\subsubsection{Overview}

Chemical vapor deposition is one of commonly used techniques for diamond growth on the silicon. Chemical vapor deposition is defined as the deposition of a solid on a heated surface from a chemical reaction in the vapor phase [Pierson, 1999]. CVD is thought as a versatile process suitable for the manufacturing of coatings, powders, fibers monolithic components. A recent paper shows a possible approach to obtain the unsymmetrical-triangle-shape nanotips shown in Figure 1.5 by applying ion beam etching (IBE) with a small angle related to the coating [Yang and Tang, 2008]. This paper concludes that, if the etching is conducted perpendicular to the coating, the coating with uniform isosceles-triangle-shape nanotips can be obtained, and that, if the etching is conducted with an angle smaller than 90 degrees related to the coating surface, 
unsymmetrical-triangles-shape nanotips can be obtained, thus resulting directional microstructure of the coating.

Microwave chemical vapor deposition (MWCVD) and hot filament chemical vapor deposition (HFCVD) are considered as the candidates for the deposition process in this research. Both techniques can be used to deposit diamond onto silicon, and the coatings obtained have similar performance. The difference in these two techniques is that the MWCVD needs 24-hour deposition, while HFCVD can accomplish the similar result in 3-4 four hours. In addition, the MWCVD equipment used in this study can only manage small samples with size of $1 \times 1 \mathrm{~cm}^{2}$, while the HFCVD equipment available can manage the samples around $1 \times 4 \mathrm{~cm}^{2}$. The small size of samples brings the difficulty to sample management and the sequent performance tests as well. Although the HFCVD has higher efficiency and the ability to manage larger samples, it has a defect that it holds relatively higher possibility to fail compared to the MWCVD. After comparing these two CVD methods, the HFCVD is chosen to be used in this study given that its defect is less harmful.

In order to fabricate diamond coating with the desired microstructure, a combination of hot filament chemical vapor deposition and ion beam etching was employed and the processes are depicted in Figure 2.1. The HFCVD is one of the commonly used CVD techniques for diamond growth. It produces radicals for diamond formation by thermal catalytic cracking of the precursors $\left(\mathrm{H}_{2}+\mathrm{CH}_{4}\right)$ at the filament at high temperatures [Sezer et al., 2001]. In the first step, diamond coatings about $20 \mu \mathrm{m}$ in thickness were grown on silicon substrates by the HFCVD. The experimental details and principle for the HFCVD can be found in [Chen et al., 2005]. IBE is a 
physical technique using individual atoms in ion beam to etch material surface into desired structures. The as-grown diamond coatings will be etched with $\mathrm{Ar}^{+}$ion beam to cut off the material with related angle with respect to the coating. The angle between ion beam and substrate tangible was set to $60^{\circ}$ in order to achieve the directional microstructure.

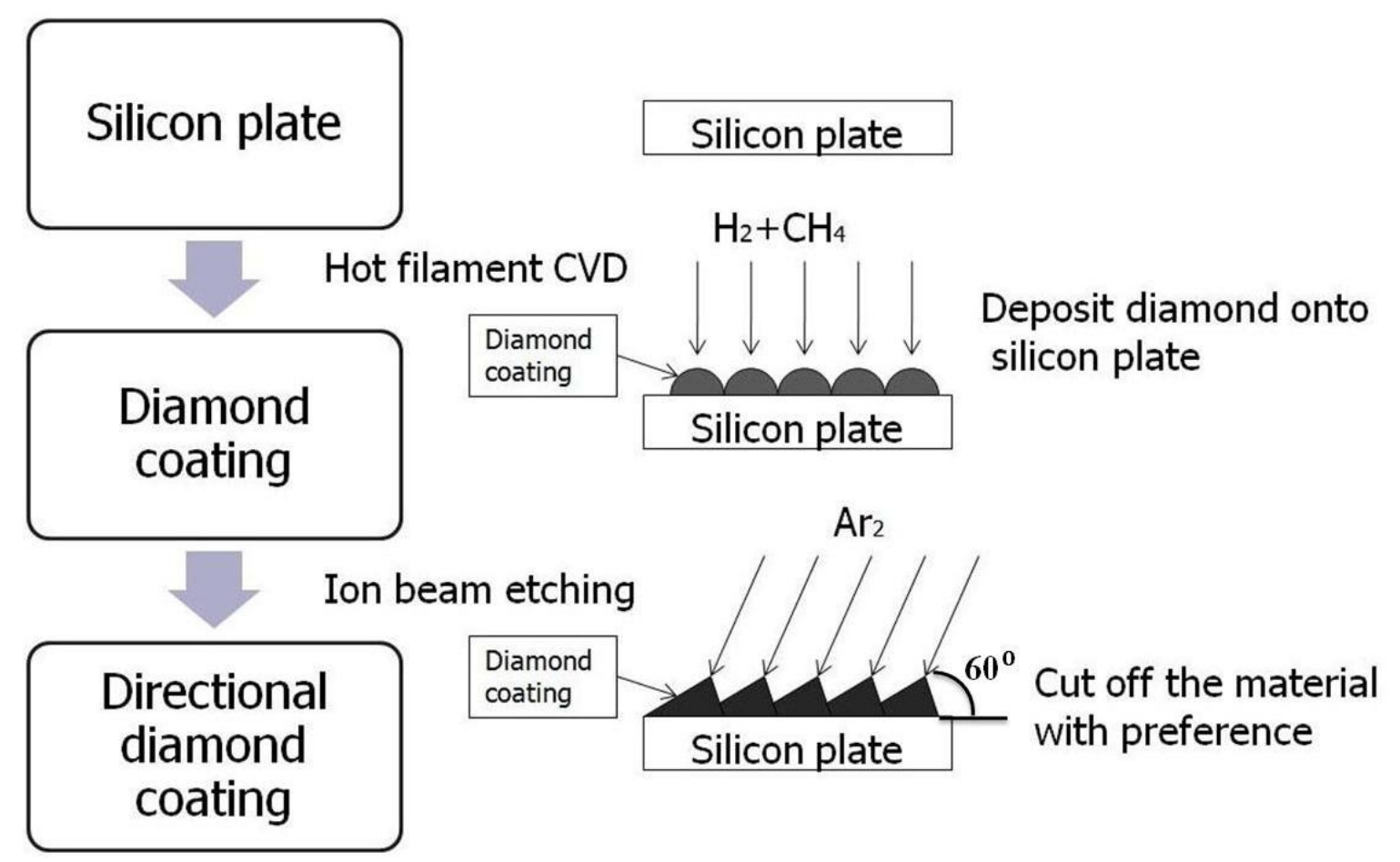

Figure 2.1 Procedure of fabricating the AF surfaces.

\subsubsection{Hot Filament Chemical Vapor Deposition}

Hot filament chemical vapor deposition is to use chemical processes energized by filament to deposit solid material onto the substrate. Specifically in this research the deposit substrate is a polished silicon plate. To improve the deposition performance, the plate was sunk in and scratched with the diamond powder-methanol solution for 2 hours by ultrasonic machine. 
The scratch on the plates is in the same size level with the diamond crystalloid, which is able to facilitate the deposition process. Then the sample was placed in the deposition chamber.

The HFCVD equipment with the deposition chamber is shown in Figure 2.2 (a) and its principle is schematically shown in Figure 2.2 (b). The sample on the substrate holder was 1 centimeter away from the filament. The inputs to the chamber were hydrogen $\left(\mathrm{H}_{2}\right)$ and methane $\left(\mathrm{CH}_{4}\right)$ gas. The chemical reaction was energized by the high temperature of the filament activated by $10 \mathrm{~mA}$ current. To ensure the desired current, the voltage was adjusted around $50 \mathrm{~V}$ to compensate the variation of the filament resistance due to the manufacturing error. By doing so, the temperature of filament was controlled to be around $1000{ }^{\circ} \mathrm{C}$ and the temperature in the reaction chamber around several hundred degrees. The operational conditions used in this study are shown in Table 2.1.
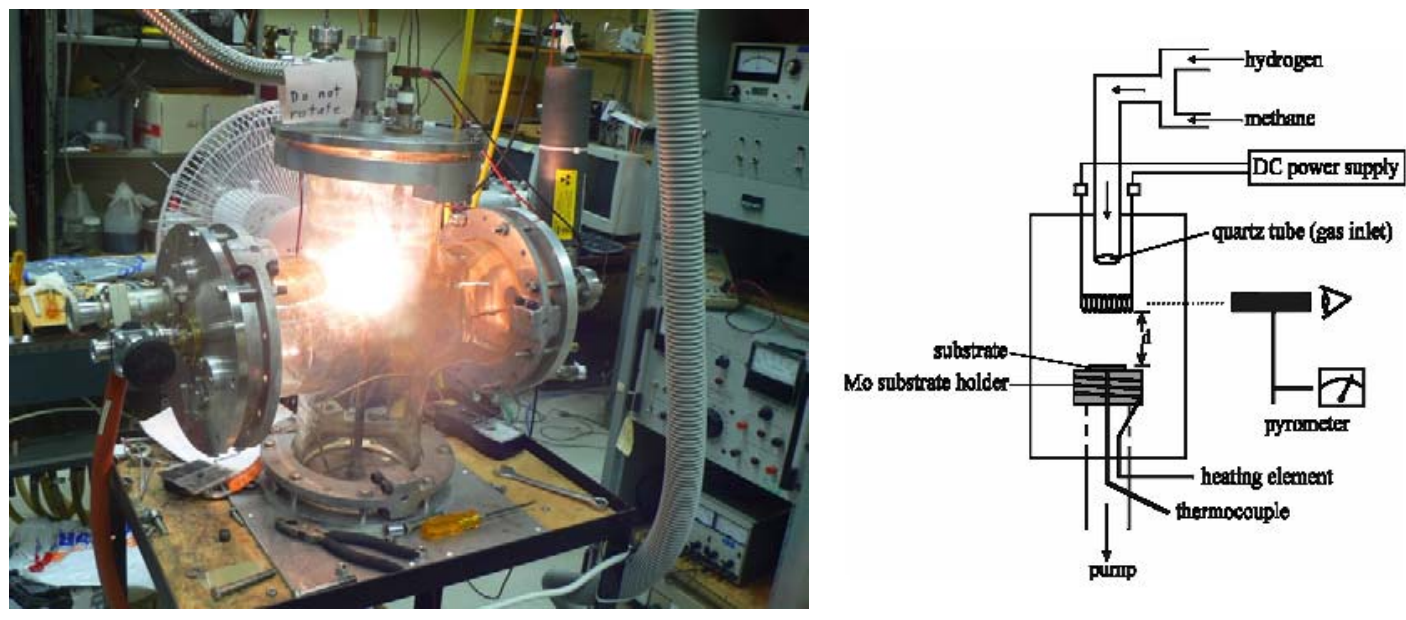

Figure 2.2 (a) HFCVD equipment and (b) Overview of HFCVD principle. 
Table 2.1 HFCVD operation conditions.

\begin{tabular}{ll}
\hline Input gas 1 & $\mathrm{H}_{2}(50 \mathrm{sccm})$ \\
\hline Input gas 2 & $\mathrm{CH}_{4}(0.6 \mathrm{sccm})$ \\
\hline Deposition time & 2.5 hours \\
\hline Air pressure in chamber & 5 Torr \\
Current on filament & $10 \mathrm{~mA}$ \\
\hline
\end{tabular}

\subsubsection{Ion Beam Etching}

Ion beam etching is a physical technique using individual atoms in ion beam to ablate the target [Norgate et al., 1974]. Ion beam etching was conducted on the diamond coating following the chemical vapor deposition process. The IBE equipment is shown in Figure 2.3 (a) and the working principle of the IBE equipment is schematically shown in Figure 2.3 (b). Specifically in this research, the substrate was the diamond coating on silicon plates, and the etching gas was the $\operatorname{argon}\left(\mathrm{Ar}_{2}\right)$ gas. Under the -800 bias voltages, individual atoms were accelerated to a high speed and collide to the coating. Some of parts of the coating were ablated off by the energized atoms. The etching angle with respect to the coating surface is related to the direction of the nanotips and the quantity of the angle decides the difference of friction coefficient between the directions of along the nanotips and against the nanotips. The bigger etching angle is supposed to induce bigger difference between the friction coefficients on two opposite directions on the friction surface. The etching angle was set to $60^{\circ}$ and $90^{\circ}$ to test the effect of the angle for the friction coefficients. The use of $90^{\circ}$ etching angle means that the product of the etching is isotropic 
friction surfaces which is normal uniform traditional surfaces. Table 2.2 summaries the IBE operation conditions.

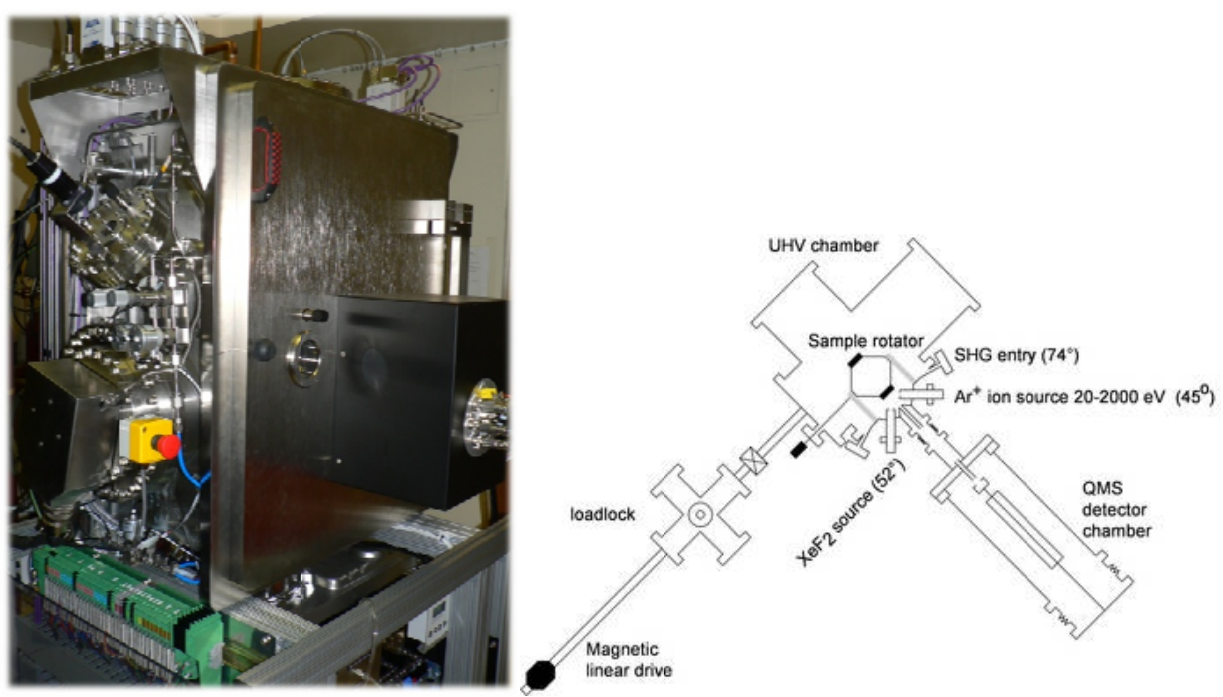

Figure 2.3 (a) IBE equipment and (b) Overview of working principle of IBE equipment.

Table 2.2 IBE operation conditions.

\begin{tabular}{ll}
\hline Substrate & Diamond coating \\
\hline Etching gas & $\operatorname{Ar}_{2}(30 \mathrm{sccm})$ \\
\hline Bias voltage & $-800 \mathrm{eV}$ \\
\hline Ion beam current & $75 \mathrm{~mA}$ \\
\hline Etching time & $1-2$ hours \\
\hline Etching angle & 60 degrees \\
\hline
\end{tabular}




\subsection{Characterization of AF Surfaces}

The etched diamond crystalloid on the coating is in the micron level, and most of single crystalloids are around 1-2 microns. To evaluate the result of fabrication, a scanning electronic microscopy (SEM, JSM 840 A) was used to image the surface morphology of the diamond coatings. As-grown diamond crystals are shown in Figure 2.4 (a), from which one can find that the crystals are well faceted and equiaxial. Figure 2.4 (b) shows the surface structure after ion beam etching. It can be seen that the equiaxial diamond crystals changed into aligned tip structure. Figure 2.5 (a) and (b) shows the similar SEM result of microstructure of AF surfaces from Yongji Tang's following research. His pictures are showed here because his SEM result shows clearer microstructure and we were using the same technologies and procedures and worked together in the same lab. The result indicates that the surface with the desired oriented nanostructure is successfully achieved.

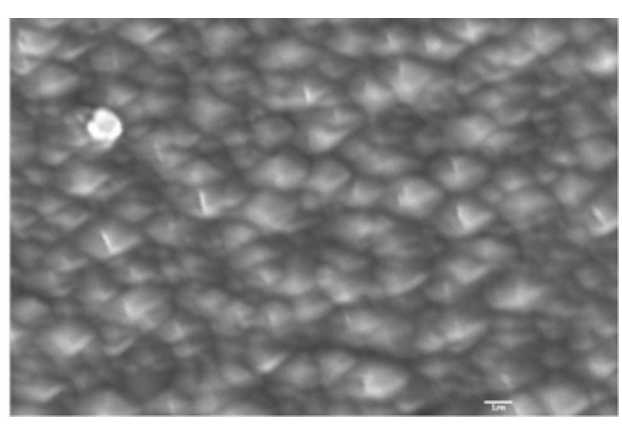

(a)

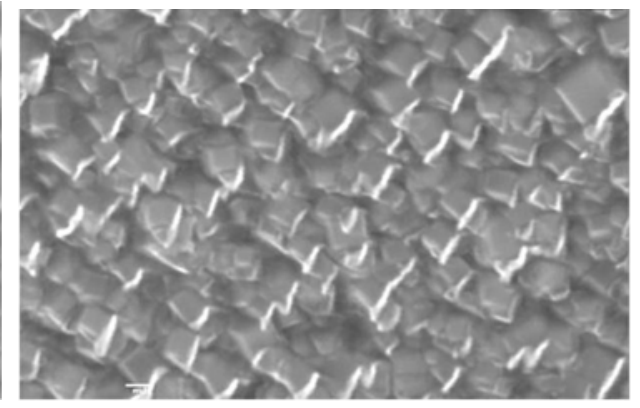

(b)

Figure 2.4 Representative SEM micrographs (top-view) of (a) the deposited diamond coating and (b) the etched diamond. 


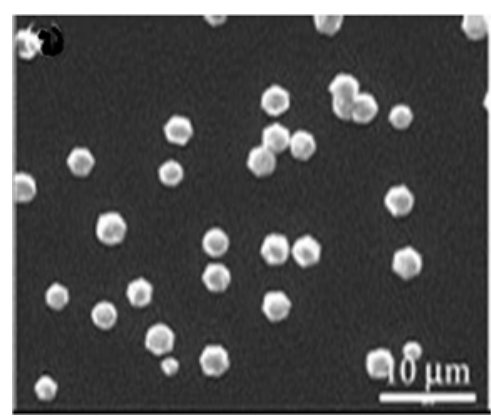

(a)

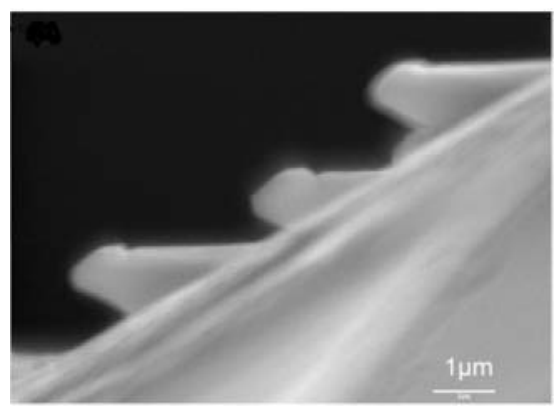

(b)

Figure 2.5 Representative SEM micrographs of (a) the deposited diamond coating and (b) the etched diamond.

In order to estimate the friction behavior of the fabricated surfaces quantitatively, ball-ondisc friction tests were carried out on a universal material testing machine manufactured by CETR shown in Figure 2.5. In these tests, a stainless steel ball with a diameter of $3 / 8^{\prime}$ was used and the test speed was at $0.5 \mathrm{~mm} / \mathrm{second}$. The normal load was maintained at $5 \mathrm{~N}$. The friction force and normal load were measured by a force sensor and the friction coefficients were calculated based on the measured values. The friction tests were performed along two directions: the diamond nanotips of each surface slide along (direction A) and against (direction B) each other. The friction coefficients were recorded respectively as shown in Figure 2.6. The average friction coefficient is 0.495 along the nanotips or namely in direction A, and is 0.432 against the nanotips or namely in direction $\mathrm{B}$, showing the anisotropic friction. 


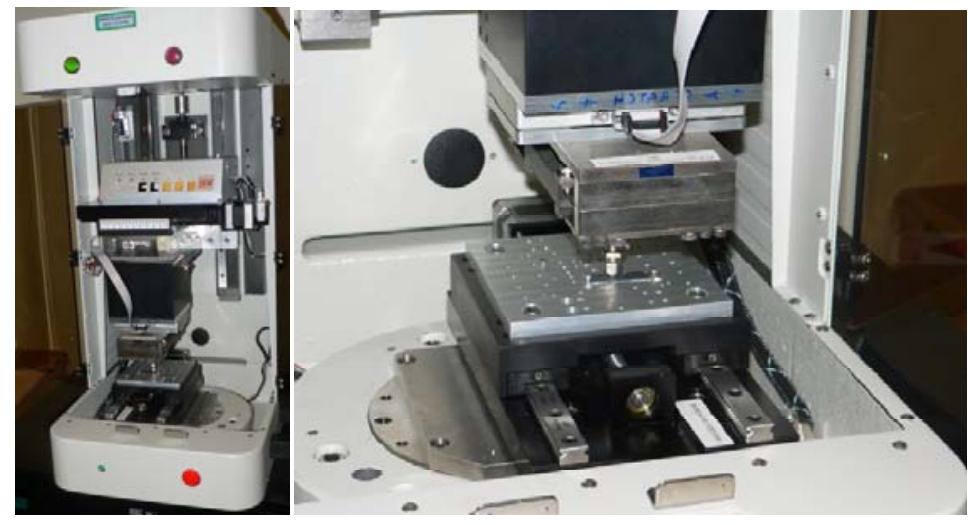

Figure 2.6 Ball-on-disk friction test equipment.

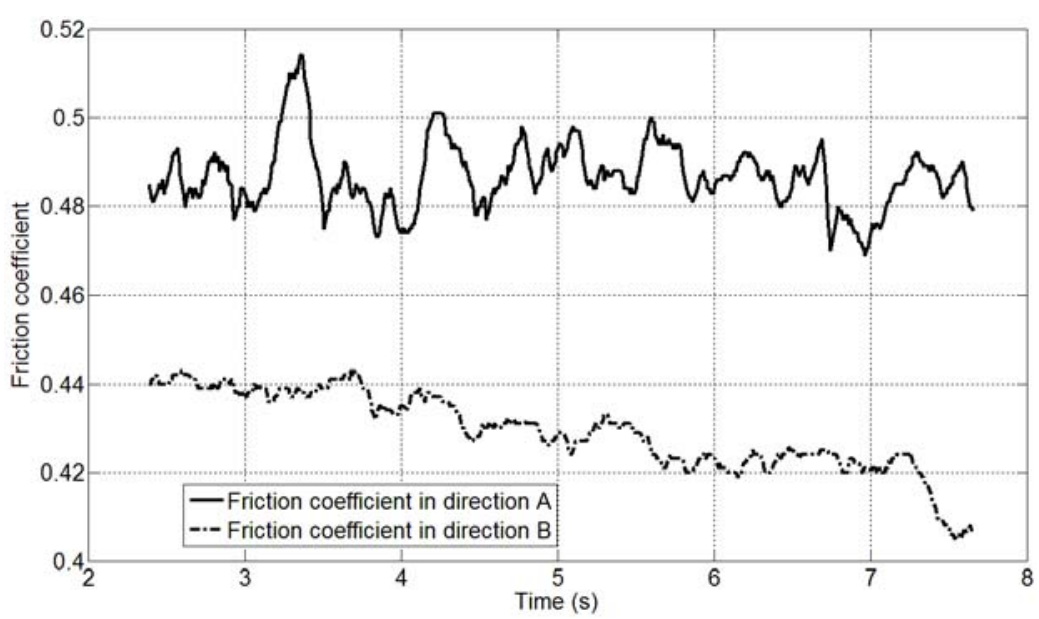

Figure 2.7 Friction coefficients in the ball-on-disk tests.

\subsection{Summary}

This chapter presents the design and fabrication of the AF surfaces as well as its characterization. At the beginning of the chapter, the selection of surface materials is discussed based on the requirement imposed on the materials for the AF surfaces. Then, the fabrication process and detailed experiments are presented, which is followed by the characterization of the AF surfaces fabricated. The results obtained from the SEM shows that the AF surfaces was 
successfully fabricated with the desired oriented nanostructure, while the ball-on-disk friction tests on the AF surfaces illustrates that the friction coefficients of the two opposite directions are different as desired. 
CHAPTER 3 PROTOTYPE OF A NOVEL PDSS ACTUATOR WITH AF SURFACES

\subsection{Introduction}

The design and fabrication of AF surfaces was discussed in the preceding chapter. In this chapter, the AF surfaces are used to prototype a PDSS actuator and its performance is then characterized. Specifically, the prototype of the novel PDSS actuator is presented in Section 3.2; and the experiment setup and data acquisition system are discussed in Section 3.3. The experiment procedure and results are described in Section 3.4 to characterize the performance of the PDSS actuators. A special attention is paid to the loading capability of PDSS actuator in Section 3.5.

\subsection{Prototype of the Novel PDSS Actuators}

By means of the AF surfaces, a novel PDSS actuator was prototyped. The schematic of the PDSS actuator prototype is shown in Figure 3.1, and a picture of the prototype is given in Figure 3.2, along with the sensor for the displacement measurements. The prototype mainly consists of a PEA, a platform, an end-effector, a supporting cylinder, a frame, and a stage. The PEA (AE0505D16, NEC/TOKIN Corp.) and the power amplifier (ENV400/ENT400, Piezosystem Jena Inc.) were chosen for the prototype, with one PEA's end mounted on the frame 
and the other end on the platform. The platform was made of aluminum, supported by the cylinder that can roll freely on the stage in order to minimize the involvement of the friction from the stage. On the platform is the end-effector with a length of $77 \mathrm{~mm}$ and a weight of $86 \mathrm{~g}$, which is made of brass. The contact surfaces of the end-effector and the platform were fabricated having 90-degree V-shape and machined to be smooth and have the roughness profile of less than 0.37 arithmetic mean deviation; and on the contact surfaces are the AF surfaces adhered firmly, as shown in Figure 3.3, forming the working surface of the actuator.

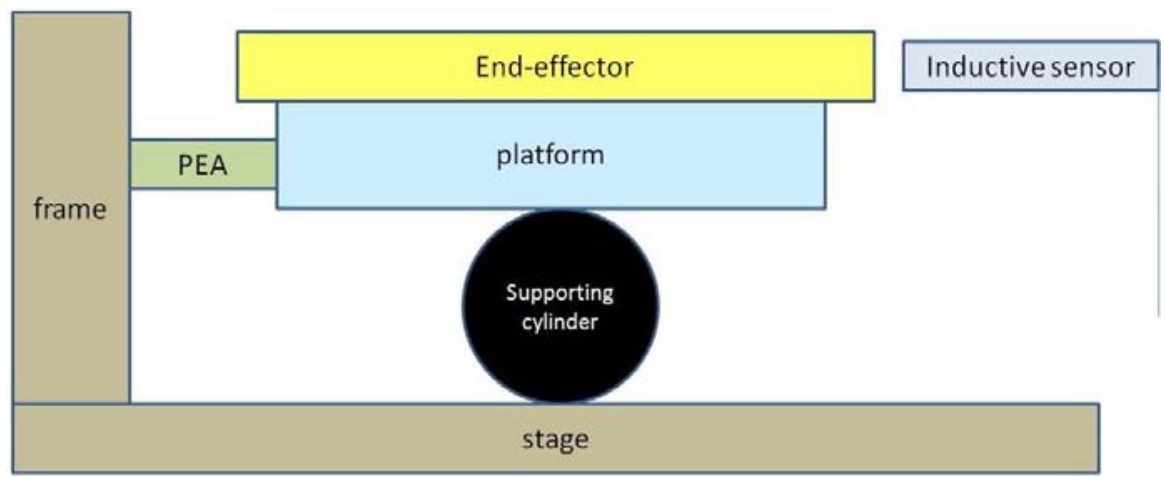

Figure 3.1 Schematic configuration of PDSS actuator prototype.

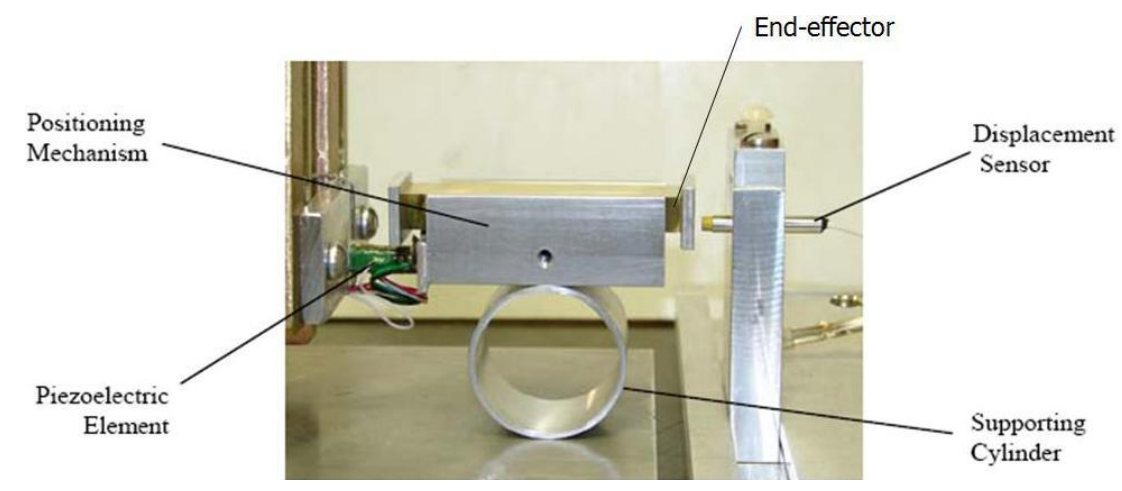

Figure 3.2 Photo of the PDSS actuator prototype. 
The supporting cylinder also named a wheel is an aluminum thin wall cylinder with 40 millimeter diameter, which is used to minimize the involvement of the friction between the platform and the stage. Both ends of the PEA were glued onto the two small aluminum plates; and these plates were screwed onto the frame and the platform, respectively. There is a small hole in the center of platform which is used to help to locate the wheel.

All the components were manufactured in Engineering Shop in the College of Engineering of the University of Saskatchewan except the PEA. The PEA is aresin coated Tolin AEO505D16 (made by NEC/TOKIN Corp.). It has the size of $5 \times 5 \times 20 \mathrm{~mm}^{3}$ and a density of $8 \times 10^{-3} \mathrm{~g} / \mathrm{mm}^{3}$

As introduced in chapter 2 , the surfaces were fabricated on the silicon substrates with a size of $1 \times 4 \mathrm{~cm}^{2}$. The end-effector and platform have V-shape surfaces with a size of $1.5 \times$ $6.5 \mathrm{~cm}^{2}$, on which the silicon plates with the AF surfaces were affixed with the double sided adhesive tape, as shown in Figure 3.3.

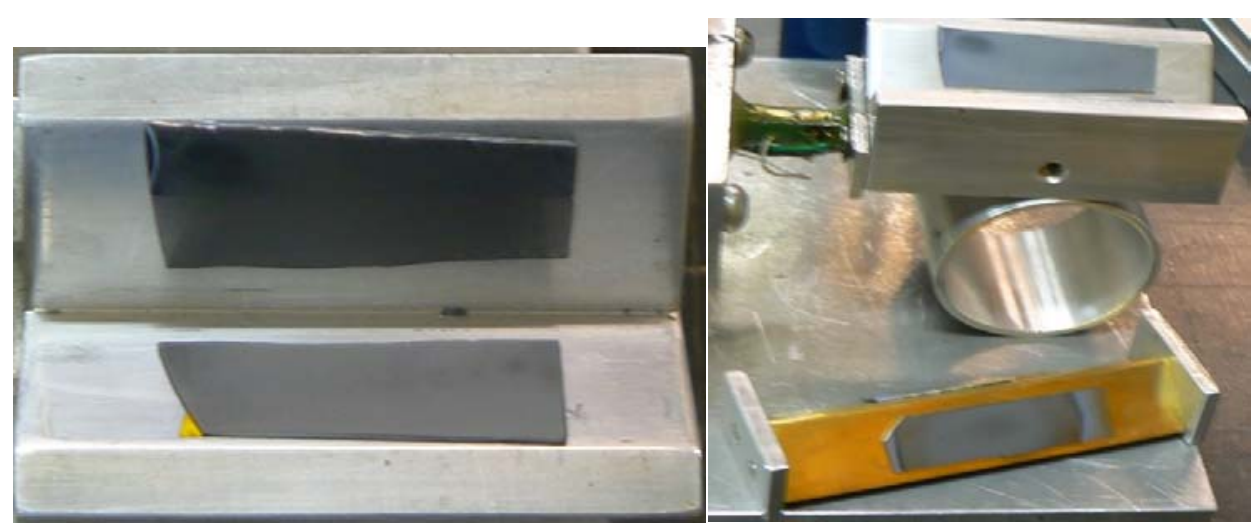

Figure 3.3 Silicon plates glued on end-effector and platform. 


\subsection{Experiment Setup}

A test rig was built to characterize the actuator performance. This test rig has the following functions: generating the voltage signal to drive the PEA; measuring the displacement of interest; recording and displaying the data for analysis. The test rig is shown schematically in Figure 3.4. The test rig mainly consists of a host computer with a specially-designed program, a controller board (DS1102, dSPACE), a power amplifier, and an inductive sensor (SMU-9000, Kaman Ins.).

The voltage signal applied to the PEA is generated in the dSPACE DS1102 controller board, which is programmed via the MATLAB SIMULINK and dSPACE ControlDesk. The ControlDesk is a software of dSPACE specially designed for real-time control applications. It allows users to specify the parameters in the application via a customer interface. Figure 3.4 shows the virtual control panel designed for this study. Once the experiment parameters are entered via the virtual control panel, by pressing the "Start" button, the DS1102 board is triggered to generate the digital signal according to the entered parameters. The generated signal is converted to analogous voltage signal by the $\mathrm{D} / \mathrm{A}$ converter, which is amplified by an amplifier and then applied to the PEA.

An inductive sensor with a resolution of $0.01 \mu \mathrm{m}$ was used to measure the displacement of the end-effector. For the measurements, an aluminum plate was mounted on the end of copper end-effecor for the reason that the sensor was calibrated by the manufacturer with aluminum plates and also the use of the aluminum plate is recommended for [SMU-9000 Use Manual, 
Kaman Instrumentation Corporation]. The signal from the sensor is filtered and then sent to the A/D converter on the DS1102 board. The digital signal obtained is then displayed on the aforementioned control panel (Figure 3.5) and recorded as well.

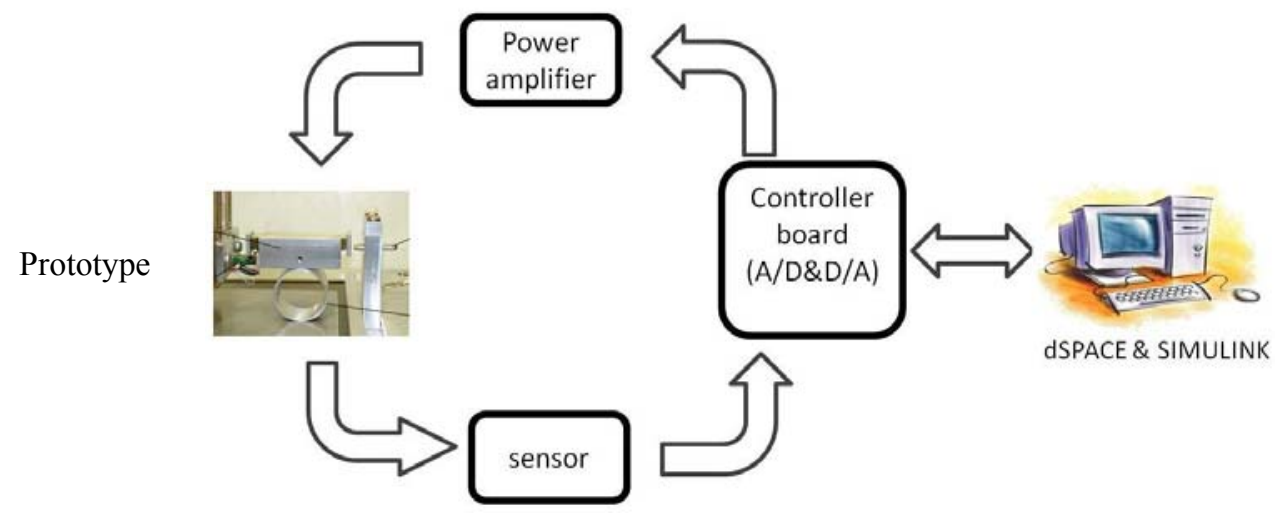

Figure 3.4 The test rig.

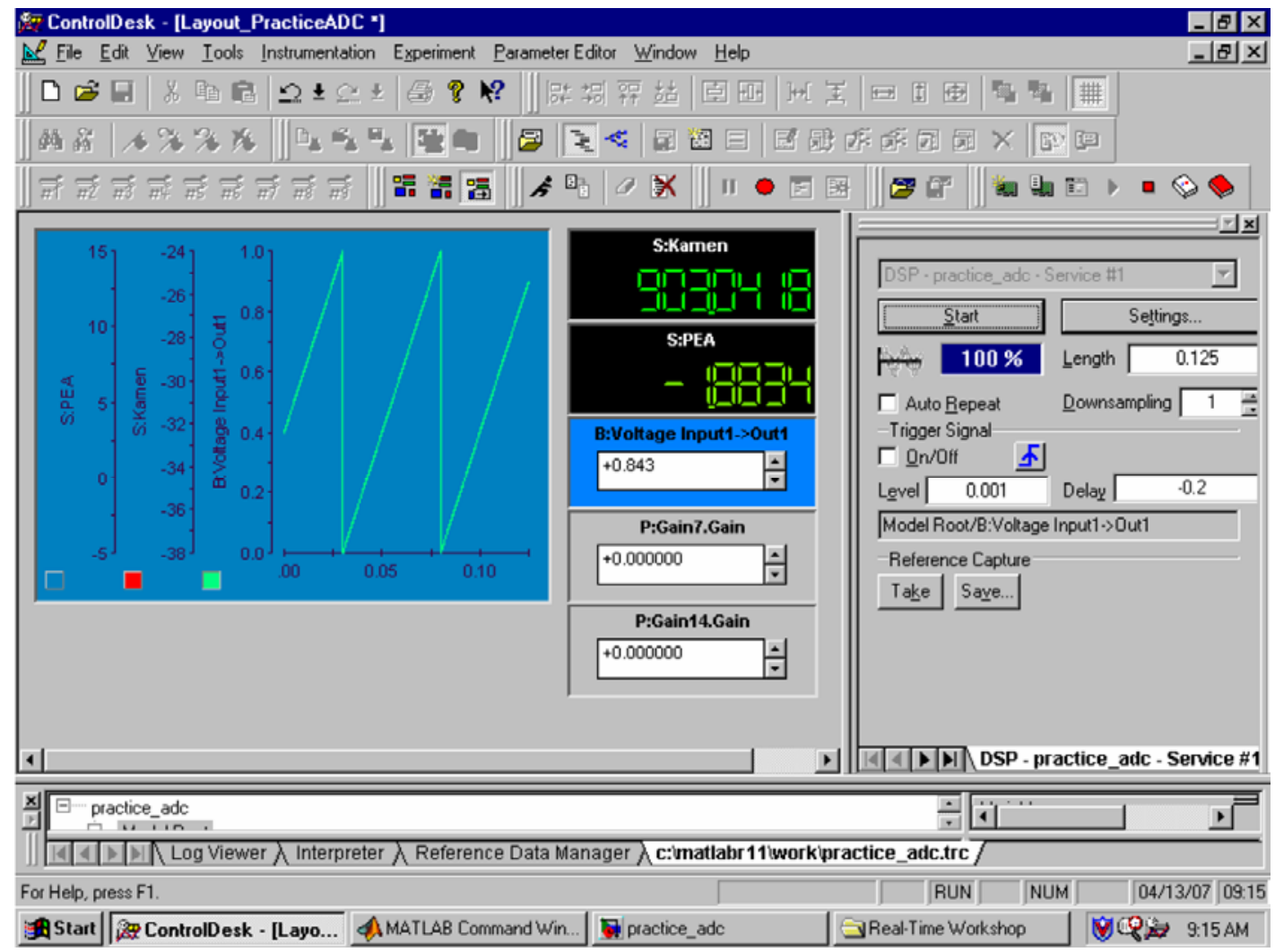

Figure 3.5 Virtual control panel of the ControlDesk in dSPACE. 


\subsection{Characterization of the PDSS Actuator Performance}

The objective of the experiments is to evaluate the effect of the AF surfaces on the PDSS actuator performance, especially its improvement compared to the performance of a PDSS actuators using IF surfaces. To achieve the objective, tests are required to see if the AF surfaces are working and achieving the desired functions and to evaluate the improvement of the PDSS actuators quantitatively. For the experiments, two types of surfaces were fabricated, i.e., AF surface and IF surface. The fabrication of the AF surfaces is presented in Chapter 2. The IF surfaces were created by etching the diamond coating with 90 degree etching angle. The reason that the diamond coating with 90-degree-angle etching was used is to make the AF surface and the IF surfaces comparable by using same material and same fabrication procedure but different etching angles. These two types of surfaces were mounted on the prototype, respectively, for the following performance characterization.

The voltages applied to the PEA were two types of sawtooth signals shown in Figure 3.6 (a) and (b), which drive the end-effector movement in two directions, respectively, namely the direction $\mathrm{A}$ and in direction $\mathrm{B}$ in the remainder of this chapter. The maximum voltage of the saw tooth waveform was set as $64 \mathrm{~V}$ and its frequency as $5 \mathrm{~Hz}$. The displacement of end-effector under these two inputs is shown in Figure 3.7 (a) for the IF surfaces. The displacements of endeffector measured in the backward direction (direction B) were all negative. To facilitate the comparison of the measured displacements in two directions, reversing the sign of the measured displacement in the direction B yields the dotted line in Figure 3.7 (b), which is the mirror imagine of the dotted line in Figure 3.7 (a) with respect to the Time-axis. For the same reason, in 
the rest of this chapter the end-effector displacements measured in the direction $\mathrm{B}$ are all reversed.

It is noted the platform could not be located perfect horizontally since the prototype was assembled manually and adjusted mechanically. This resulted in the end-effector moving forward and backward with different speeds. The reason is due to the influence of the gravity if end-effector is not located horizontally or unbalanced. After being aware of the effect of the unbalanced platform, the position of the wheel and the PEA was adjusted to minimize its effect until the measured displacements in two directions were close in an acceptable level. Figure 3.7 (b) shows the measured displacements after this adjustment.

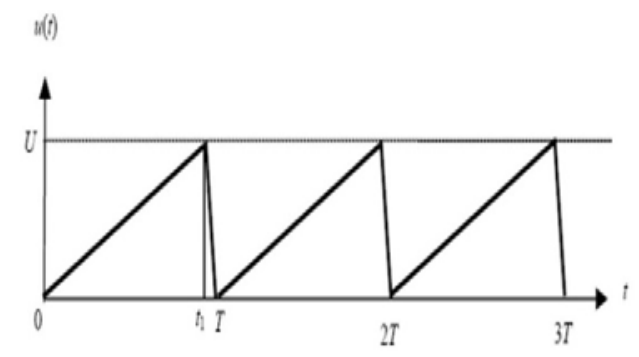

(a)

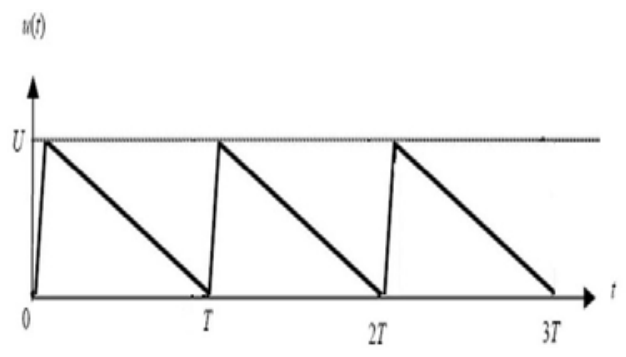

(b)

Figure 3.6 Voltage signals used in driving the PDSS actuator: (a) forward and (b) backward. 


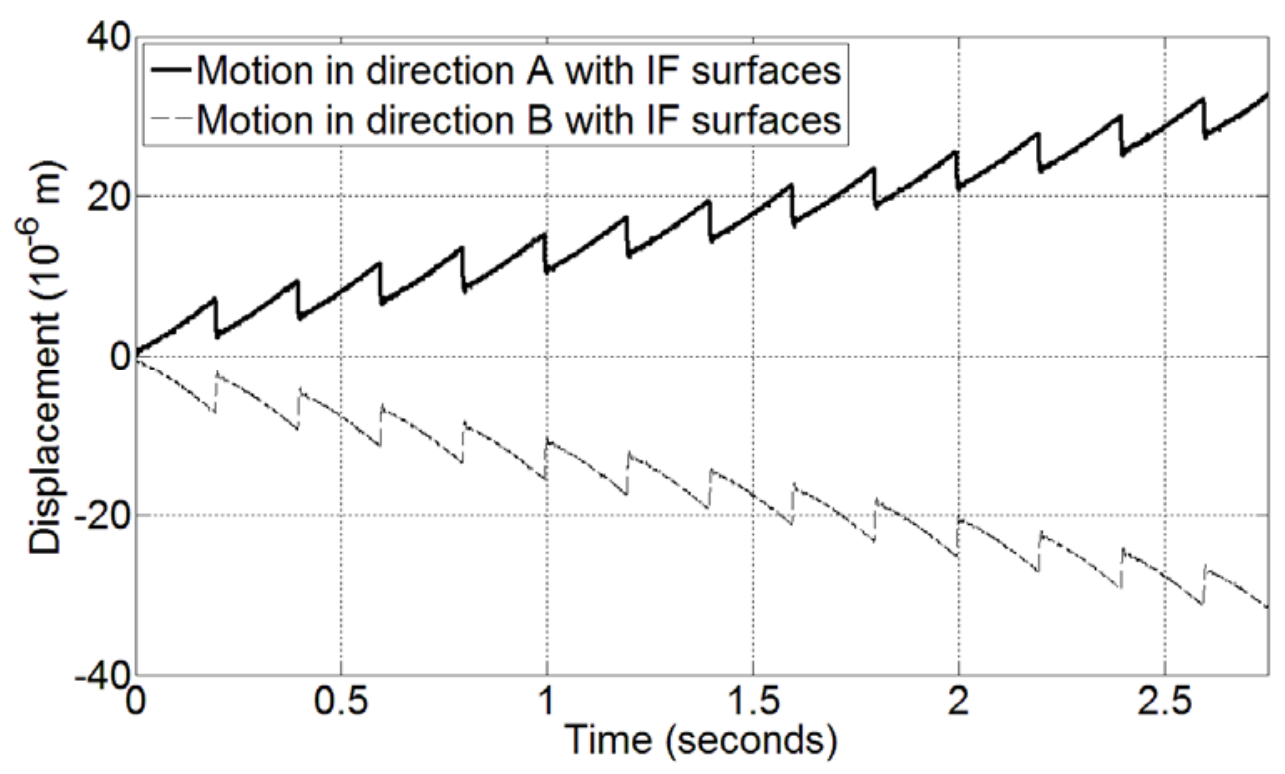

(a)

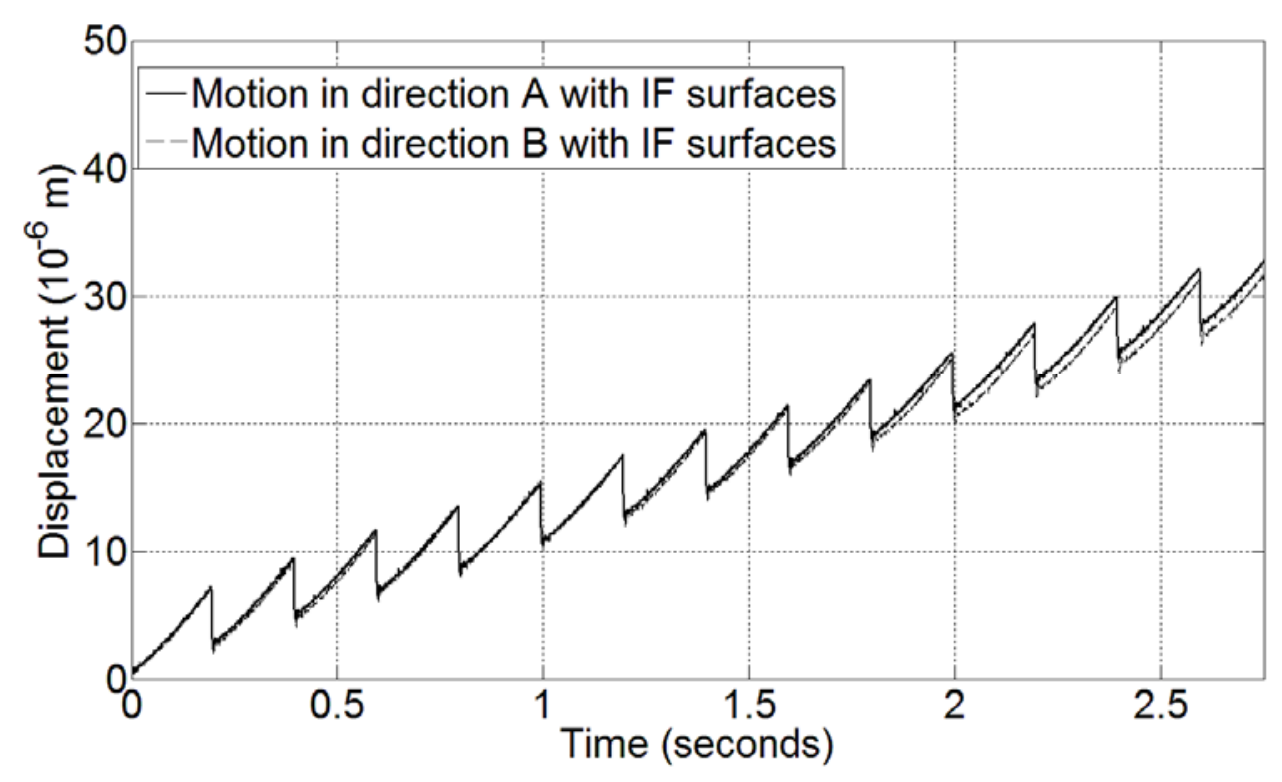

(b)

Figure 3.7 Comparison of the measured displacements of the end-effector driven by the PDSS actuator with IF surfaces. 
Once the end-effector is adjusted to be horizontal, the AF surfaces were affixed onto the end-effector and platform for tests. The same voltages (Figure 3.6) were applied to the PEA; and the displacements of the end-effector in two directions were measured. The measured displacements are presented in Figure 3.8, in which the solid line is the displacement of endeffector in direction A and the dotted line is the one in direction B. It can be seen that the endeffector moved much faster forward than backward, indicating the different frictions in both directions. Specifically, if closely looking at on one step (e.g. the first step shown in Figure 3.8), one can see that, in the stick phase, the end-effector and the platform moved together, which means the surfaces were able to provide the friction required for stick in both directions. However, in the slip phase the sliding distances, denoted by $S_{0 f}$ and $S_{0 b}$, respectively, in Figure 3.8, depends on the direction; $S_{0 f}<S_{0 b}$. This suggests that the friction in one direction is less than that in the other direction.

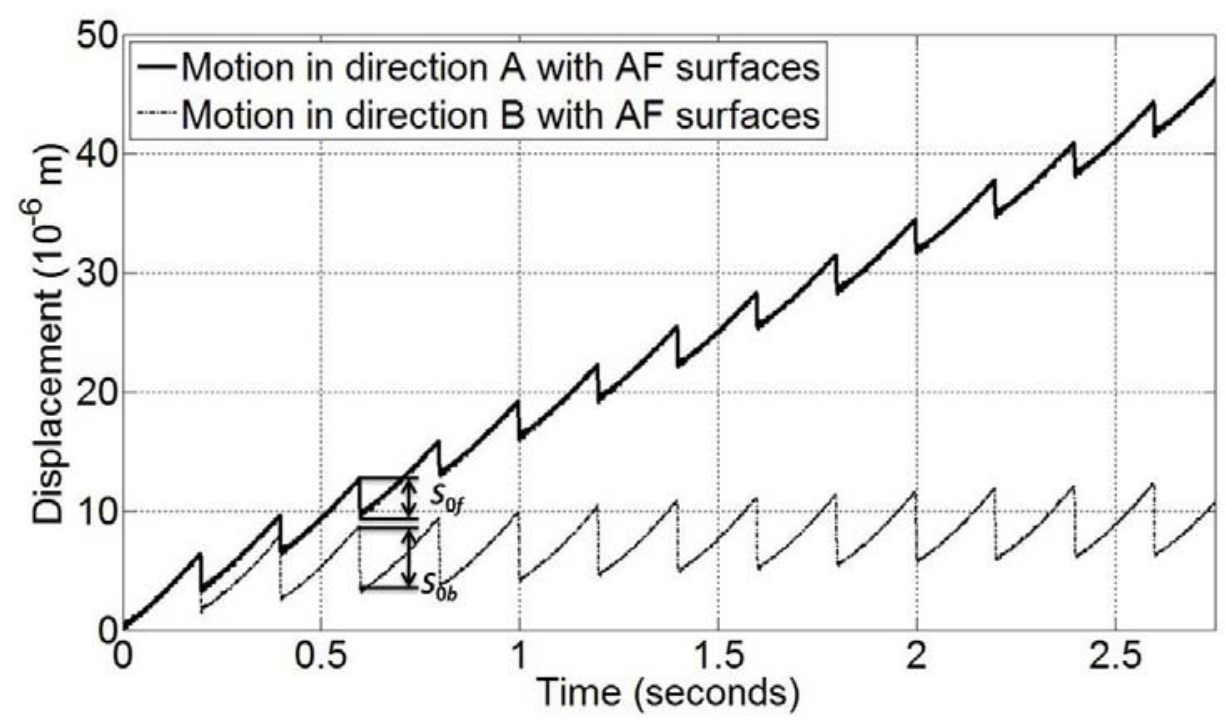

Figure 3.8 Comparison of the measured displacements of the end-effector driven by the PDSS actuator with AF surfaces. 
In order to observe the improvement of the actuator with the AF surfaces over the one with IF surfaces, the measured displacements of the end-effector, driven forward by both actuators, respectively, were shown together in Figure 3.9. The black and blue lines are the displacement of PDSS actuators with AF surfaces, and the red and pink lines are the displacement of PDSS actuators with IF surface. The average speeds in direction A on AF and IF surfaces were evaluated as $16.0 \mu \mathrm{m} / \mathrm{s}$ and $10.0 \mu \mathrm{m} / \mathrm{s}$, respectively. The faster speed of the actuator with AF surfaces is due to the fact that, in the slip phase, the AF surfaces provides less friction, compared to the IF surfaces, thereby resulting in larger sliding distance. The difference between the black and blue lines does not indicate the improvement of PDSS actuator performance using AF surfaces, but the difference between black and red lines does that.

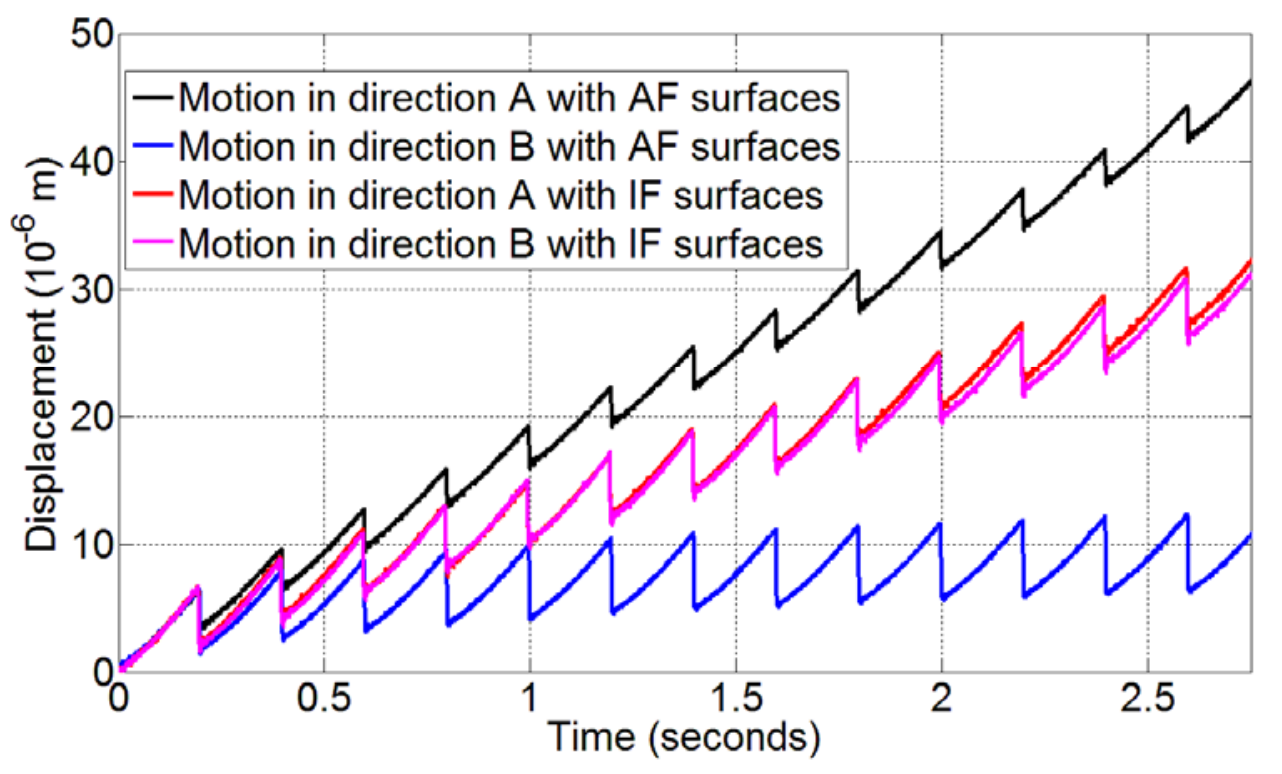

Figure 3.9 Experimental result of performance of PDSSA using two surfaces. 
In order to analyze the improved efficiency or the enhanced performance quantitatively, a concept of step efficiency of a PDSS actuator (denoted by $\eta_{e}$ ) is introduced in this study, which is defined by

$$
\eta_{e}=\frac{s-S_{0}}{s} \times 100 \%
$$

where $S$ is the displacement of forward motion in the stick phase, $S_{0}$ the sliding displacement of backward motion in the slip phase, both of which are illustrated in Figure 1.1. Using the above equation, the step efficiencies for the results shown in Figure 3.9 were evaluated. The results obtained are shown in Table 3. It can be seen that the average step efficiency of PDSS actuators with traditional IF surfaces on both directions was detected as $32.78 \%$, and taking it as a reference, the step efficiency of novel PDSS actuators on direction A were improved to $50.65 \%$.

\begin{tabular}{|c|c|c|}
\hline \multicolumn{2}{|c|}{ Table 3.1 Efficiency improvement using AF surfaces } \\
\hline & Efficiency (\%) & Improved Efficiency \\
\hline Motion in direction A with AFS & 50.65 & 17.87 \\
\hline Motion in direction B with AFS & 21.72 & -11.06 \\
\hline Motion in direction A with IFS & 32.61 & Average Efficiency: \\
\hline Motion in direction B with IFS & 32.95 & 32.78 \\
\hline
\end{tabular}




\subsection{Loading Capability Performance Improvement}

The loading capability was tested by a force sensor (Load Cell Transducer-MB50, Interface Inc.). As shown in Figure 3.10, the force sensor was placed in front of the end-effector to block its motion. Thus, under the aforementioned sawtooth wave input signals driving the endeffector forward, the end-effector kept pushing the sensor with its output force which was measured by the sensor. The input signal was the sawtooth waveform with a maximum voltage of $64 \mathrm{~V}$, and its frequency was set to $5 \mathrm{~Hz}$ and $20 \mathrm{~Hz}$. The experiment was conducted on the PDSS actuators with IF surfaces and AF surfaces, respectively, for comparison.

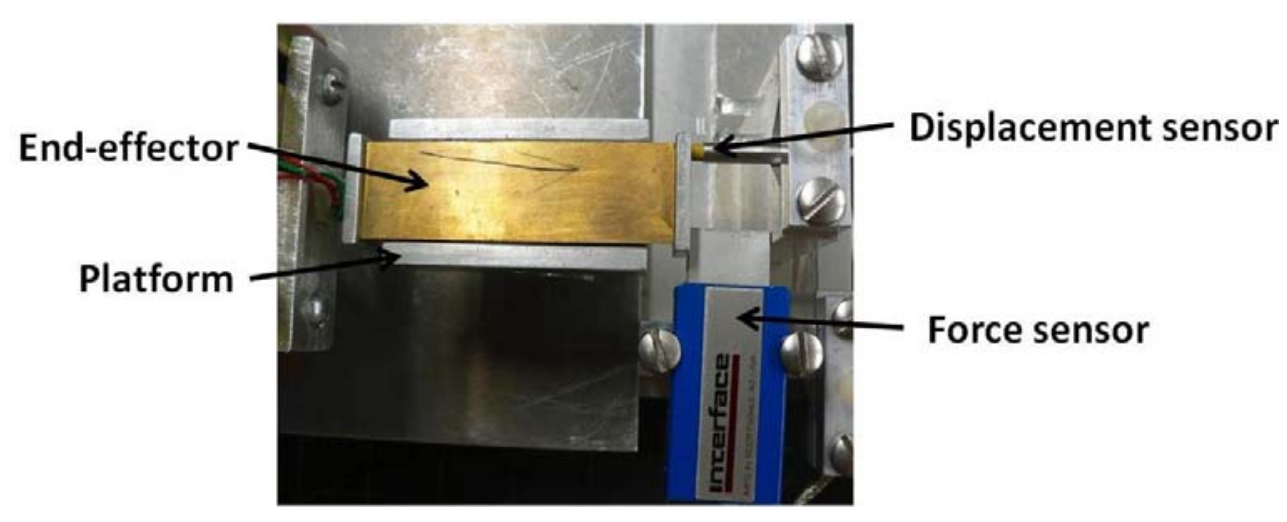

Figure 3.10 Setup of the force sensor.

Since the input is the sawtooth wave signal, the output force is also in the sawtooth wave form. Therefore, the maximum output force is used to measure the loading capability of the PDSS actuators. The maximum output forces of actuators with IF and AF surfaces under $5 \mathrm{~Hz}$ and $20 \mathrm{~Hz}$ input were shown in Figure 3.13. It can be seen that the one using AF surfaces shows 
a larger maximum output force than the one using IF surfaces, which implies that the loading capability of PDSS actuator was improved by using AF surfaces on it.

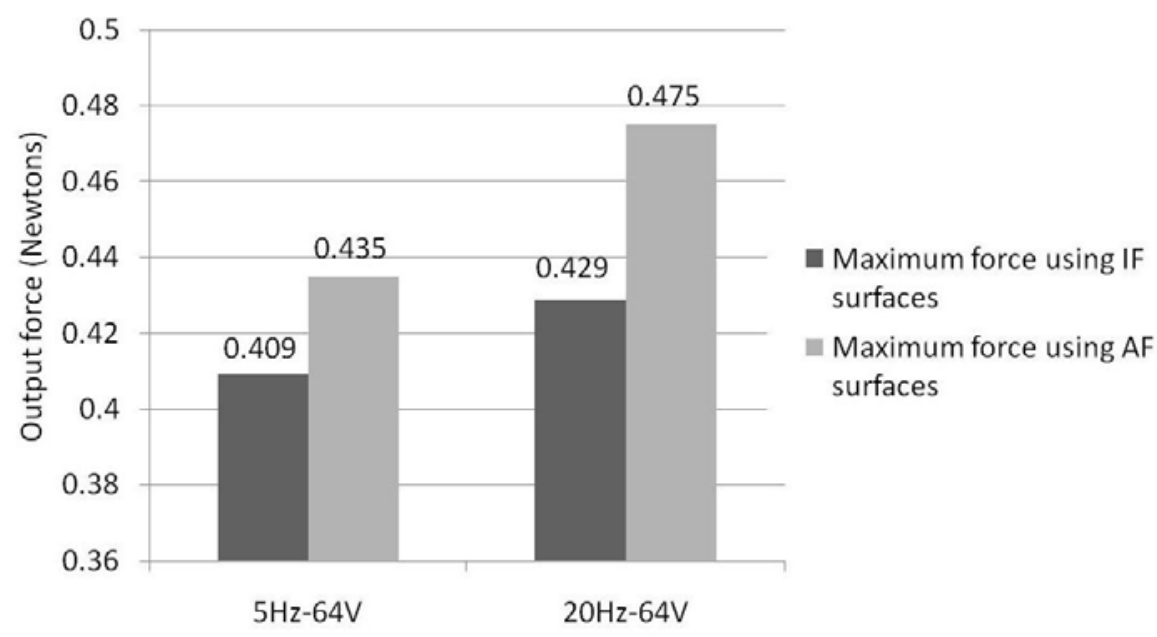

Figure 3.11 Measured maximum output force of PDSS actuators with IF surfaces and AF surfaces under different inputs.

\subsection{Summary}

In this chapter, experiments conducted to characterize the performance of the novel PDSS actuators are presented. At first, the experiment setup and test rig is described. Secondly, experiment conditions are presented and the experimental result indicates that the AF surfaces are working and the step efficiency of PDSS actuator was improved from $32.78 \%$ to $50.65 \%$ by using AF surfaces. Thirdly, experimental results show that the loading capability of the PDSS actuator is improved by using the AF surfaces. Under $64 \mathrm{~V} / 5 \mathrm{~Hz}$ input signal, the maximum loading is increased from $0.409 \mathrm{~N}$ to $0.435 \mathrm{~N}$; under $64 \mathrm{~V} / 20 \mathrm{~Hz}$ input signals, the maximum loading is increased from $0.429 \mathrm{~N}$ to $0.475 \mathrm{~N}$. 


\section{CHAPTER 4 MODELING OF THE PEA DYNAMICS}

\subsection{Introduction}

Piezoelectric actuators are commercially available for producing extremely small displacements. If a PEA is used to drive a positioning mechanism, the combined dynamics is approximated empirically by that of a second-order system. However, the rationale for such an approximation is lacking in the literature, thus leaving some issues unaddressed, such as the scope of and the error associated with the approximation. This chapter presents such a rationale and its experimental verification. Some previous PEA models and their properties are reviewed in Section 4.2. The dynamics of the piezo-actuated positioning systems is modeled in Section 4.3. Experiments and simulations are presented in Section 4.4, along with their results for the verification of the model.

\subsection{Previous Model of the PEA}

Piezo-actuated positioning systems have found applications in many fields of technology and research, such as semiconductor manufacture and precision machining. In these applications, the behavior of the positioning system has proven to be of importance for its design and/or control. By considering the piezoelectric actuator as a standalone system, Goldfarb and 
Celanovic [Goldfarb et al., 1997] developed a model, based on the generalized Maxwell resistive capacitor, to represent the static hysteresis that is always observed in PEAs. Despite the presence of the non-linear hysteresis, the dynamics of the PEA was observed to have simple second-order linear characteristics [Goldfarb et al., 1997]. Adriaens et al. [Adriaens et al., 2000] elaborated on this model by including a first-order hysteresis effect and by modeling the PEA as a distributed parameter system. The authors concluded that if the positioning system was designed well, the aforementioned second-order approximation was a good approximation of the system dynamics. Usually, the PEA is used to drive a mass-stage, as shown in Figure 4.1. The combination of the PEA and the mass-stage is modeled as a simple mass-spring-damping system, and the interaction force between the PEA and the mass stage, denoted by $F s$, is given by [Adriaens et al., 2000]

$$
m_{s} \ddot{y}+c_{s} \dot{y}+k_{s}=F_{s}
$$

where $y$ represents the displacement of the mass-stage, $m_{s}$ the mass of the mass-stage, $c_{s}$ the mass-stage damping coefficient and $k_{s}$ the mass-stage stiffness. These terms are also used in the Figure 4.1 where $F_{p}$ is the transformed force from the electrical side of PEA.

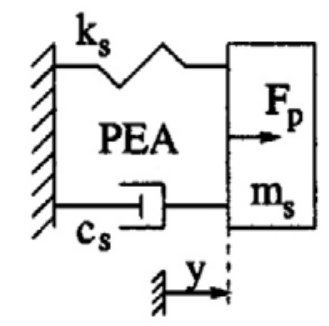

(a)

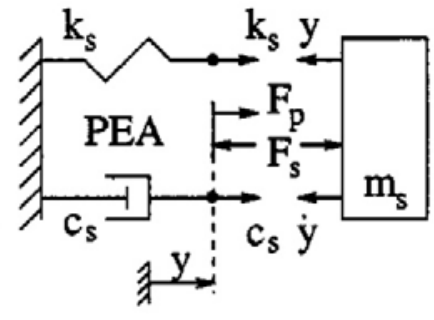

(b)

Figure 4.1 (a) Total system of PEA and the mass-stage; (b) Force analysis of the system of a PEA and a mass-stage [Adriaens et al., 2000]. 
By ignoring the hysteresis effect and other nonlinearities, a combined system of a PEA and a mass is modeled as a second order system, which is given by

$$
m_{g} \ddot{y}+c_{g} \dot{y}+k_{g}=T u
$$

where $u$ is the voltage signal applied to the PEA, $m_{g}$ the mass of the PEA and the mass-stage, $c_{g}$ the damping ratio of PEA and mass-stage, $k_{g}$ the stiffness of PEA and mass-stage, $T$ the electrical-mechanical transformer ratio. The values of these parameters are dependent on the geometries and masses of both the PEA and the mass stage.

This empirical approximation has been also widely employed in the applications where the system dynamics is concerned, for example, the ones reported in [Zhang et al., 2006; Liu et al., 2001]. However, the rationale for such an approximation is lacking in the literature. This also leaves some issues unaddressed, such as the scope of and the error associated with the approximation. This purpose of this chapter is to fill the void by providing with such a rationale and its experimental verification.

\subsection{Modeling the Dynamics of Piezo-Actuator Positioning Systems}

Consider a PEA shown in Figure 4.2, which is used to drive a positioning mechanism with a mass of $M$. Figure 4.2 also includes an element with an infinitesimal thickness of $d y$. The forces on both sides of the element are $N(y, t)$ and $N(y+d y, t)$. Under the action of these forces, 
the element has moved a distance $v(y, t)$ from its equilibrium position at $y$, as measured from the fixed end of the PEA. Summing the forces on the element, one has

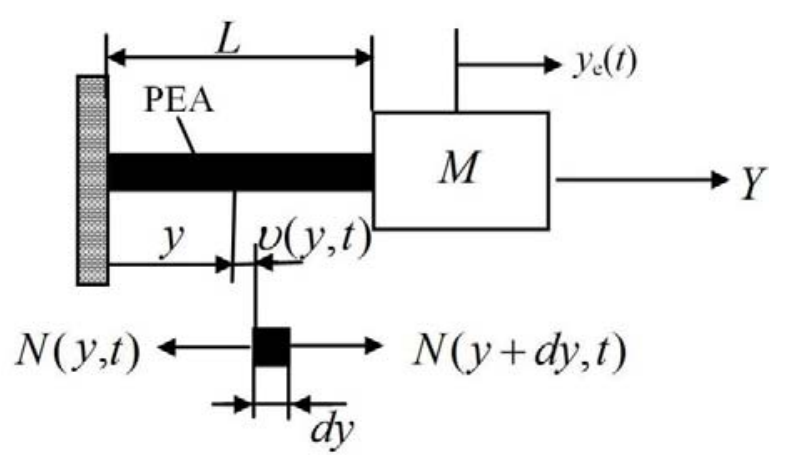

Figure 4.2 Piezo-actuated positioning systems.

$$
\rho A d y \frac{\partial v^{2}(y, t)}{\partial t^{2}}=N(y+d y, t)-N(y, t)=\frac{\partial N(y, t)}{\partial y} d y
$$

where $t$ is time, and $\rho$ and $A$ are the mass density and cross-sectional area of the PEA. Under the assumption that the PEA is viscoelastic, one can use the Kelvin-Voigt model to describe the relationship between the stress and the strain in the material, i.e.,

$$
\sigma(y, t)=E \varepsilon(y, t)+\eta \frac{\partial \varepsilon(y, t)}{\partial t}
$$

where $\sigma(y, t)$ and $\varepsilon(y, t)$ are the stress and strain, and $E$ and $\eta$ the Young's modulus and viscous coefficient of the PEA material, respectively. Using the definition of stress and strain, i.e., $\sigma(y, t)=N(y, t) / A$ and $\varepsilon(y, t)=\partial v(y, t) / \partial y$, the following equation can be obtained from 
Equations (4.3) and (4.4)

$$
\rho \frac{\partial^{2} v(y, t)}{\partial t^{2}}=E \frac{\partial^{2} v(y, t)}{\partial y^{2}}+\eta \frac{\partial^{3} v(y, t)}{\partial y^{2} \partial t}
$$

From (4.5), it is seen that $v(y, t)$ is governed by a partial differential equation. To seek a solution for such an equation, the boundary conditions need to be used. Based on the fact that the displacement at the fixed end of the PEA is zero and the fact that the force in the PEA at the other end equals to the inertial force of the positioning mechanism, the boundary conditions can be expressed mathematically by

$$
\begin{gathered}
v(0, t)=0 \\
N(L, t)=-\left.M \frac{\partial^{2} v(y, t)}{\partial t^{2}}\right|_{x=L}
\end{gathered}
$$

By means of the assumed mode method, the solution to (4.5) is given by a linear combination of assumed modes with different frequencies, i.e.,

$$
v(y, t)=\sum_{j=1}^{\infty} \phi_{j}(y) q_{j}(t)
$$

where $\phi_{j}(y)$ is the normal mode function and $q_{j}(t)$ is the mode coordinate. Substituting (4.8) to (4.5) and utilizing the boundary conditions given in (4.6) and (4.7), one has the following normal mode function [Palm, 2007] 


$$
\phi_{j}(y)=B \sin \left(p_{j} y\right)
$$

where $B$ is a constant, depending on the initial condition of the system; and $p_{j}$ is governed by the following equation

$$
p_{j} L \tan \left(p_{j} L\right)=\frac{m}{M}
$$

in which $m=\rho A L$, which is the mass of the PEA. For given values of $L, m$, and $M$, one can solve for $p$ from (4.10) numerically. It should be noted that $p$ has an infinite number of values, corresponding to the orders of modes. If a voltage is applied to the PEA, by means of the aforementioned assumed modes, one has the mode coordinates governed by

$$
\ddot{q}_{j}(t)+\frac{\eta p_{j}^{2}}{\rho} \dot{q}_{j}(t)+\frac{E p_{j}^{2}}{\rho} q_{j}(t)=D \frac{E p_{j}^{2}}{\rho} u(t)
$$

where $u(t)$ is the voltage applied to the PEA and $D$ is a coefficient, depending on the PEA materials used. By taking the Laplace transformation of (4.11), the transfer function of $Q_{j}(s) / U(s)$ can then be derived

$$
\frac{Q_{j}(s)}{U(s)}=\frac{D \omega_{j}^{2}}{s^{2}+2 \xi_{j} \omega_{j} s+\omega_{j}^{2}}
$$

where $\xi_{j}$ and $\omega_{j}$ are the damping ratio and natural frequency of the $j$ th-mode coordinate, and are given by $\omega_{j}=p_{j} \sqrt{\frac{E}{\rho}}$ and $\xi_{j}=\frac{\eta p_{j}}{2} \sqrt{\frac{1}{\rho E}}$, respectively. 
In the present study, the displacement of the positioning mechanism driven by the PEA, denoted by $y_{e}(t)$ in Figure 4.2, is of particular interest. It is noted that $y_{e}(t)$ is the same as the displacement of the endpoint of PEA, i.e., the motion $v(y, t)$ at $y=L$. Thus, $y_{e}(t)$ is also characterized by (4.8) and (4.9) with $y$ replaced by $L$, and (4.11) or (4.12). On this basis, $y_{e}(t)$ can be represented as the parallel second-order subsystems that are described by (4.12) with the numerator replaced by $D \omega_{j}{ }^{2} \phi_{j}(L)$ (or $H \omega_{j}{ }^{2} \sin \left(p_{j} L\right)$, where $H=D B$ ). The block diagram of the representation is shown in Figure 4.3.

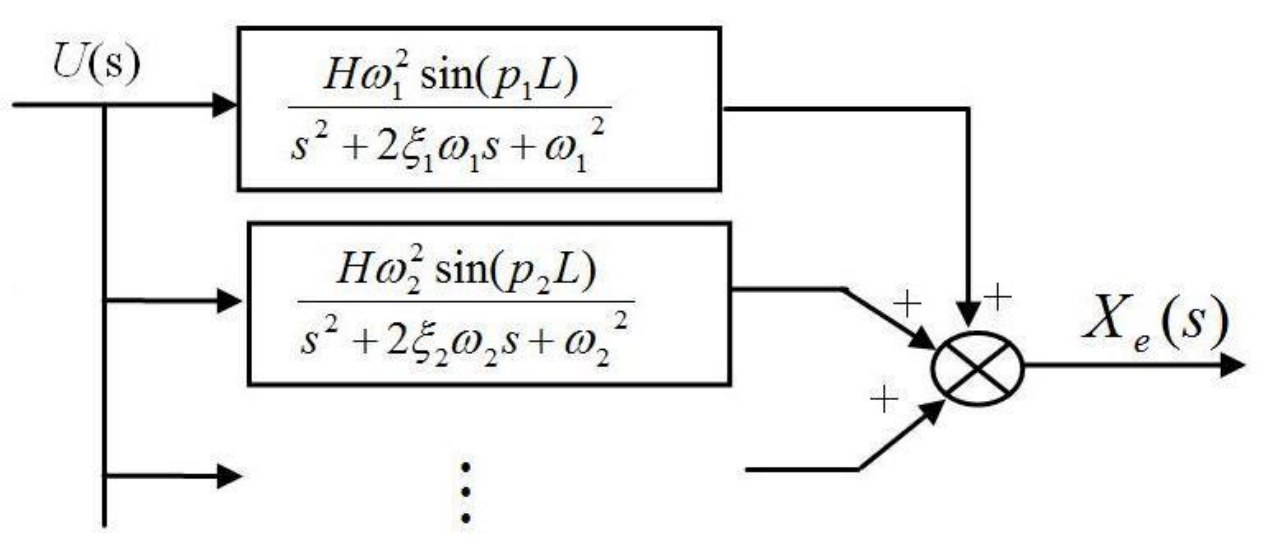

Figure 4.3 Block diagram representing the displacement of the positioning mechanism.

It is noticed from the solutions to (4.9) and (4.10) that $\phi_{j}(L)$ decreases as the mode order (i.e., $j$ ) increases, depending on the ratio of the positioning mechanism mass to the PEA mass (i.e., $M / m$ ). Figure 4.4, for example, illustrates the magnitude ratio of the second mode to the first one as a function of the mass ratio. It can be seen that, as the mass ratio increases, the magnitude of the second mode becomes much less than that of the first one. As a result, the displacement of 
the positioning mechanism (i.e., $\left.y_{e}(t)\right)$ is dominated by the first mode. Thus, the second-order subsystem corresponding to the first mode can be used to approximate the dynamics of positioning system. Meanwhile the error involved in the approximation can be evaluated from other second-order subsystems, given by

$$
E(s)=U(s) \sum_{j=2}^{\infty} \frac{H \omega_{j}^{2} \sin \left(p_{j} L\right)}{s^{2}+2 \xi_{j} \omega_{j} s+\omega_{j}^{2}}
$$

where $E(s)$ is the Laplace transformation of the error $e(t)$. If the mass ratio is a relatively small number, one may consider the use of two or more second-order subsystems for improved accuracy, and the error is also governed by (4.13) with, for example, $j=2$ replaced by $j=3$ for the use of two second-order subsystems. Therefore, for a given piezo-actuated positioning system, the use of (4.13) allows one to rigorously choose an appropriate approximation for the system dynamics based on the desired accuracy.

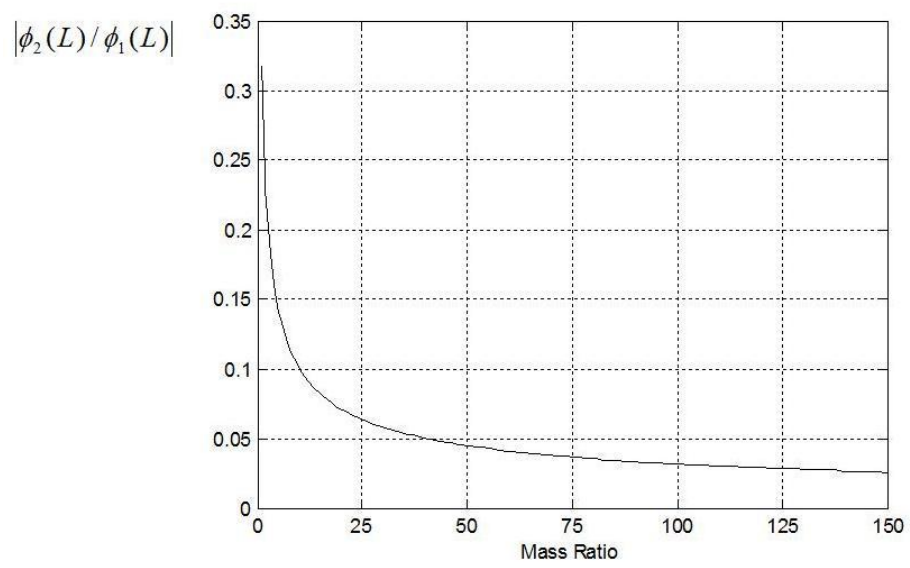

Figure 4.4 Graphical representation of $\left|\emptyset_{2}(\mathrm{~L}) / \emptyset_{1}(\mathrm{~L})\right|$. 


\subsection{Experiment and Simulation Results}

The piezo-actuated positioning system used for this experiments presented in the chapter is shown in Figure 4.5, along with a sensor for displacement measurements. Experiments of the system step responses were designed and conducted on this system to investigate the errors involved in the approximations of the system dynamics. In particular, step voltages of $48.2 \mathrm{~V}$, 64.2 V, and 75.4 V were used to excite the positioning system with a mass ratio of 24.7, 47.7, 87.5, 115.8, and 138.8, respectively; and its displacement responses were recorded. Meanwhile, the displacements were also simulated by using different approximations.

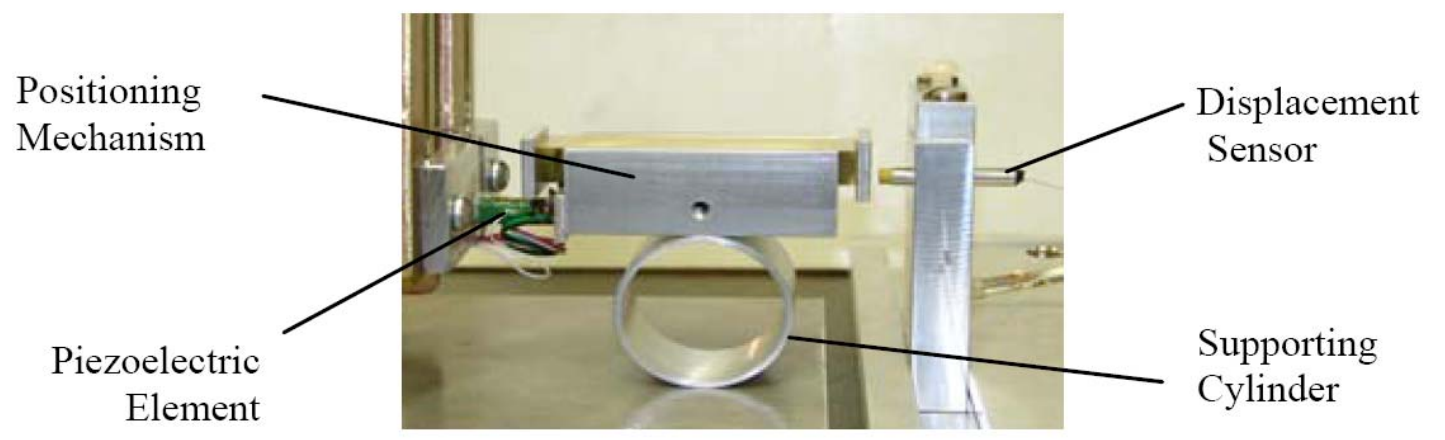

Figure 4.5 Piezo-actuated positioning system.

To measure the difference between the measured displacements and the model predictions, the following error index $(E I)$ was used

$$
E I=\sqrt{\frac{\sum_{i=1}^{n}\left(M D_{i}-S D_{i}\right)^{2}}{(n-1)}}
$$


where $M D$ is the measured displacement, $S D$ the simulated displacement, and $n$ the number of samples. A part of the results obtained in this study are shown in Figures 4.6 and 4.7. Specifically, Figure 4.6 shows the measured and simulated displacements for a mass ratio of 138.8. The solid lines represent the measured displacements, which were used for determining the parameters of the second order system, i.e., $H, \omega$, and $\xi$. Their values were found to be $4.93 \times 10^{-7} \mathrm{~m} / \mathrm{V}, 6527 \mathrm{rad} / \mathrm{s}$, and 0.2083 , respectively. The MATLAB programs used to identify these three parameters are shown in Appendix 1. Using these values, the step responses were simulated by using one second-order approximation; and the results are shown in dash lines in Figure 4.6. By using (4.14), the values of $E I$ for the results shown in Figure 4.6 were calculated to be $0.766 \times 10^{-6}, 0.632 \times 10^{-6}, 0.453 \times 10^{-6} \mathrm{~m}$ for $75.4 \mathrm{~V}, 64.2 \mathrm{~V}$, and $48.2 \mathrm{~V}$, respectively, illustrating the appropriateness of the use of one second-order approximation for the system dynamics for a mass ratio of 138.8 . Figure 4.7 shows the errors involved in one second-order approximation as a function of the mass ratio for an applied voltage of $48.2 \mathrm{~V}$, in which the points on a solid line were determined from the measurements and the dash line was obtained based on simulations. The MATLAB program used to derive this figure is shown in Appendix 2 . Specifically, the points on the solid line were determined from (4.14), in which $M D$ is the measured displacement and $S D$ is the simulated displacement by means of one second-order approximation; and the dash line was also obtained from (4.14), but with the term of (MD-SD) evaluated from (4.13). A good agreement is seen between the errors determined from the experiments and the simulations, which shows the promising of (4.13) in the characterization of the errors associated with the approximations of the system dynamics. Besides, it is also seen 
that the errors decreases with the increase in the mass ratio. Most important here is that the use of (4.13) make it possible to quantify these errors.

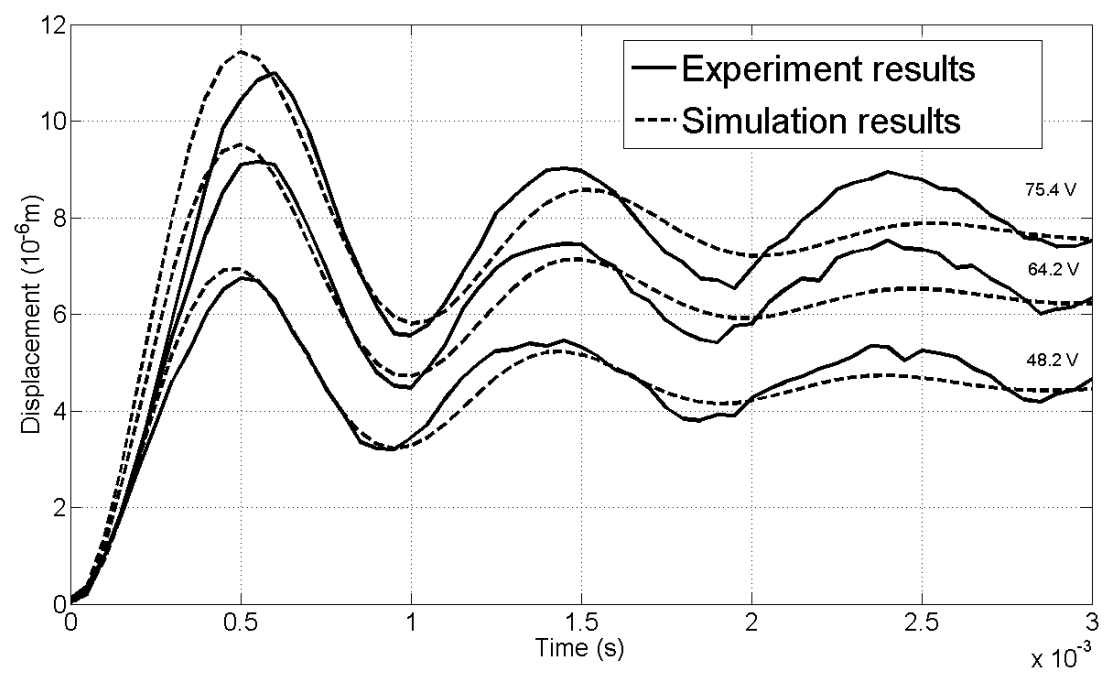

Figure 4.6 Measured and simulated step responses for a mass ratio of 138.8.

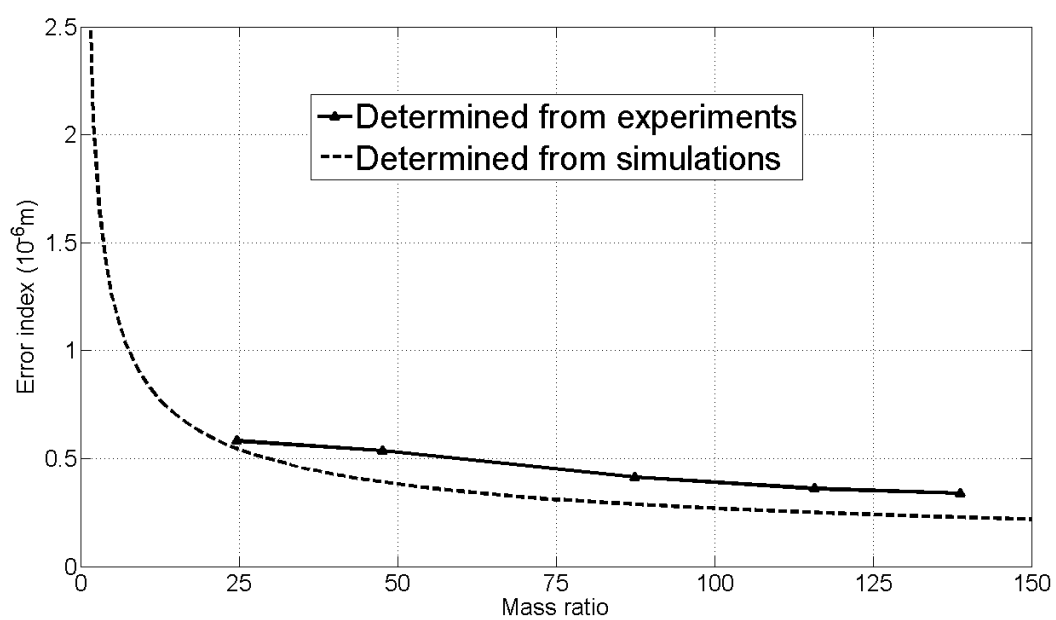

Figure 4.7 Errors involved in the second-order approximations for an applied voltage of $48.2 \mathrm{~V}$. 


\subsection{Summary}

The dynamics of a piezo-actuated positioning system is of importance for its design and/or control, which is approximated empirically by using a second-order system in the literature. The chapter provides a rationale for such an approximation and a method to quantify the error associated with the approximation. The effectiveness of the method was illustrated experimentally on the prototype of a piezo-actuated positioning system. 


\section{CHAPTER 5 MODELING OF THE NOVEL PDSS ACTUATORS}

\subsection{Introduction}

Piezoelectric-driven stick-slip actuators have drawn extensive attention for many applications, but the research in this area is still in its early stage and many issues remain to be addressed. One key issue is the representation of the dynamic displacement of the end-effector. Modeling the motion of the end-effector is still a challenging work because of the complexity of the dynamics of PEA and the representation of the presliding friction. Although some work has been done in this area, none of previous work is able to represent the anisotropic friction behavior appearing on AF surfaces. This chapter focuses on developing a model for the novel PDSS actuators. Specifically, several presliding friction models are discussed and a modified presliding friction model which is used to represent the anisotropic friction is presented in Section 5.2. Based on the presliding friction model and PEA model, a PDSS actuator model, which can model the motion of end-effector driven by the novel PDSS actuator, is presented in Section 5.3. The parameters in this PDSS actuator model are identified experimentally in Section 5.4.1 and the model is verified in Section 5.4.2. 


\subsection{Modeling the Presliding Friction}

One of the most important steps in the development of a model for PDSS actuators is the representation of the friction between the end-effector and the movable platform. Friction is universally inevitable in all mechanical systems. It resists the relative motion of bodies in contact. In control engineering, friction is an important issue which has been attracting a lot of attention. A number of friction models have been developed, but most of them focused on some specific applications since a general friction model is impossible to develop [Olsson et al., 1998]. Each of friction models for control application is able to represent some of the important friction behaviours, such as Striebeck friction, Coulomb friction, viscous friction, and presliding shown in Figure 5.1.

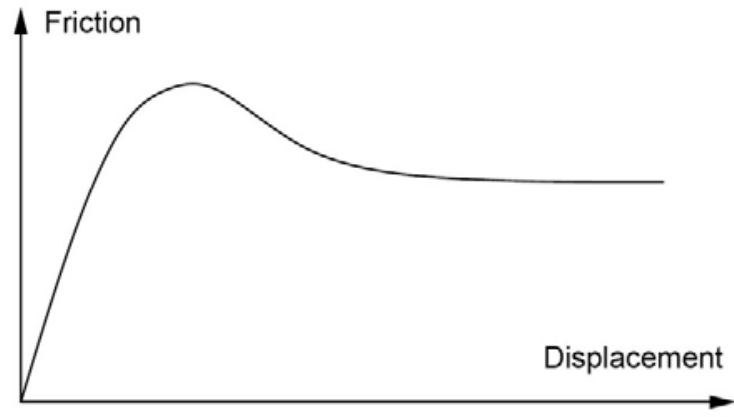

(a)

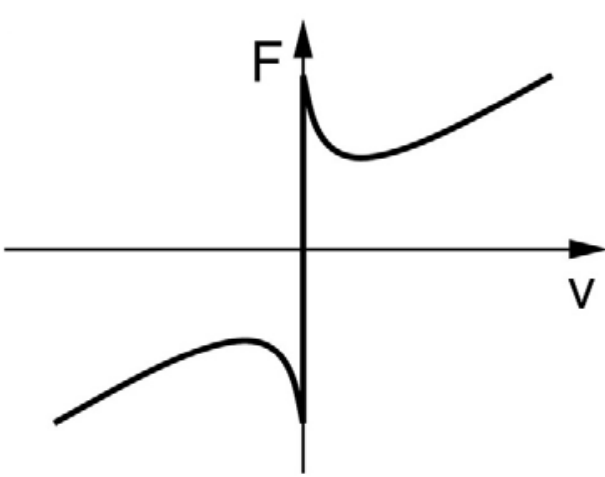

(b)

Figure 5.1 Friction behaviors: (a) The relation between friction and displacement as found by Robinowicz (b) shows how the friction force may decrease contimuously from thestatic friction level [Olsson et al., 1998]. 
For the PDSS actuator, the relative motion between end-effector and the platform is usually very small (from several nanometres to several microns). The friction occurred in such small motion is denominated by the presliding displacement [Rabinowicz, 1951]. Presliding friction is referred to the friction happening in the motion prior to the fully developed slip. The displacement of presliding friction usually extends less than a few micrometers. In this regime, characteristics of the friction differ from the one in other friction regimes [Dupont et al., 2002]. Dahl [Dahl, 1968] was the first to model the friction in presliding for control applications; the basic idea behind his model, and a subsequent generalization known as the LuGre model [Canudas de Wit et al., 1995] and elastoplastic model [Dupont et al., 2000] as well, is that the displacement $x$ is decomposed into two components; elastic (reversible) and plastic (irreversible), denoted by $z$ and $w$, respectively. And the friction is then defined as a function of $z$ and other parameters (e.g. $\dot{z}, \dot{x})$.

Dahl friction model was developed for the purpose of simulating control systems and its starting point were the stress-strain curve in classical solid mechanics [Dahl, 1968; Dahl, 1975]. Dahl modeled the stress-strain relationship using the following differential equation, in which $x$ is the displacement, $F_{f}$ the friction force, and $F_{c}$ the Coulomb friction force.

$$
\frac{d F_{f}}{d x}=\sigma\left(1-\frac{F_{f}}{F_{c}} \operatorname{sgn}(\dot{x})\right)^{\alpha}
$$

where $\sigma$ is the stiffness coefficient and $\alpha$ is a parameter which determines the shape of the stressstrain curve. Although Dahl friction model does not capture the Stribeck effect, nor the stiction, it has still been widely used for its capability of capturing many other aspects of friction and 
holding the advantage of simple expression as well [Philip and Dahl, 1976; Walrath, 1984; Ehrich and Krishnaprasad, 1992].

In the time domain, the Dahl model is expressed by

$$
\frac{d F_{f}}{d t}=\frac{d F_{f}}{d x} \frac{d x}{d t}=\frac{d F_{f}}{d x} \dot{x}=\sigma\left(1-\frac{F_{f}}{F_{c}} \operatorname{sgn}(\dot{x})\right)^{\alpha} \dot{x}
$$

For the case $\alpha=1$ the Equation (5.2) becomes

$$
\frac{d F_{f}}{d t}=\sigma \dot{x}-\frac{F_{f}}{F_{c}}|\dot{x}|
$$

Suppose the friction takes the form of $F_{f}=\sigma z$, then the model can be written as

$$
\begin{gathered}
F_{f}=\sigma z \\
\frac{d z}{d t}=v-\frac{\sigma|\dot{x}|}{F_{c}} z
\end{gathered}
$$

LuGre friction model was developed based on the bristle interpretation of friction [Canudas de Wit et al., 1995]. The model has the form

$$
\begin{gathered}
\frac{d_{z}}{d_{t}}=\dot{x}-\sigma_{0} \frac{|\dot{x}|}{g(\dot{x})} z \\
F_{f}=\sigma_{0} z+\sigma_{1}(v) \dot{z}+f(\dot{x})
\end{gathered}
$$


where $z$ denotes the average bristle deflection. The parameter $\sigma_{0}$ is the stiffness of the bristles, and $\sigma_{1}(v)$ the damping. After the linearization and standard parameterization [Olsson et al., 1998], the LuGre Friction model has the following general expression:

$$
\begin{gathered}
F_{f}=\sigma_{0} z+\sigma_{1} \dot{z}+\sigma_{2} \dot{x} \\
\frac{d_{z}}{d_{t}}=\dot{x}-\sigma_{0} \frac{|\dot{x}|}{g(\dot{x})} z \\
g(\dot{x})=F_{C}+\left(F_{b}-F_{C}\right) e^{-\left(\frac{\dot{x}}{v 0}\right)^{2}}
\end{gathered}
$$

where $F_{C}$ denotes the Coulomb friction, $F_{b}$ the break-away friction, $\sigma_{2}$ the viscous friction coefficient, $g(\dot{x})$ a function to describe the Stribeck effect and $v 0$ the Stribeck velocity.

As the extension of Dahl model, LuGre model includes most of the important friction behaviours and has been used in many applications [Haessig and Friedland, 1991; Olsson, 1996]. LuGre model captures the Stribeck effect, Coulomb friction, viscous friction and presliding displacement. This model can also be used to represent the rate dependent friction phenomena such as varying break-away force and frictional lag.

This friction model was extended by Depont et al., resulting in the elastoplastic friction model [Dupont et al., 2000; Dupont et al., 2002]. In this model, a breakaway displacement, denoted by $z_{b}$, was introduced by assuming that only the elastic displacement occurs if $z<z_{b}$. On this basis, the presliding displacement is considered happening in two successive stages: the elastic displacement stage and the mixed elastic and plastic displacement stage, in which the rate equations are given by 


$$
\left\{\begin{array}{l}
\dot{x}=\dot{z} \\
\dot{W}=0
\end{array}\right.
$$

for the elastic displacement stage(i.e. $\left.z<z_{b}\right)$ and

$$
\dot{x}=\dot{z}+\dot{w}
$$

for the mixed elastic and plastic displacement stage (i.e., $z>z_{b}$ until $\dot{z}=0$ or $\dot{x}=\dot{w}$ )

The friction is then represented by using the following expression

$$
F_{f}=\sigma_{0} z+\sigma_{1} \dot{z}+\sigma_{2} \dot{x}
$$

where $\sigma_{0}, \sigma_{1}$, and $\sigma_{2}$ are positive coefficients. $z$ is governed by

$$
\dot{z}=\dot{x}\left(1-\alpha(z) \frac{z}{z_{s}}\right)
$$

in which $z_{S}$ is the steady-state value of $z$ for a given $\dot{x}$ and $\alpha$ is a function of $z$. And $\alpha(z)$ is given in the following piecewise continuous function 


$$
\alpha(z)=\left\{\begin{array}{cc}
0 & |z| \leq z_{b} \\
\frac{1}{2} \sin \left[\frac{z-\left(\frac{z_{S}+z_{b}}{2}\right)}{z_{S}-z_{b}}\right] \\
1 \\
0 \text { if } \operatorname{sgn}(\dot{x}) \neq \operatorname{sgn}(z) & |z| \geq z_{S}(\dot{x})
\end{array}\right\} \quad \text { if } \operatorname{sgn}(\dot{x})=\operatorname{sgn}(z)
$$

The elastoplastic friction model includes more friction aspects and is able to represent express the friction behaviour more precisely. The drawback of the model is the complicated form used, and also too many parameters are involved in this model.

Some other friction models have been developed, including the Reset Integrator model [Haessig and Friedland, 1991], Bristle model [Haessig and Friedland, 1991], Bliman and Sorine models [Bliman and Sorine, 1991; Bliman and Sorine, 1993; Bliman and Sorine, 1995], which also represent some specific friction aspects. As indicated in the previous research [Kang, 2007], the LuGre friction model is most suitable in this stick-slip application, while Dahl model is not sufficient and elstoplastic model is too complicated in form and other friction models focus on other aspects. In this research, LuGre friction model is adopted for its simple form, capability of representing friction aspects in this stick-slip application. In order to represent the directional friction behaviour occurring on AF surfaces, the LuGre model is modified, which is introduced in the following section.

In the elastic phase of the presliding displacement, the displacement consists of only elastic component, i.e., z. Therefore, one has $\dot{x}=\dot{z}$ and the equation (5.8) is reduced to

$$
F_{f}=\sigma_{0} z+\left(\sigma_{1}+\sigma_{2}\right) \dot{z}
$$


where $\left(\sigma_{1}+\sigma_{2}\right)$ is a constant and this part can be denoted simply by a $\sigma_{1}$

$$
F_{f}=\sigma_{0} z+\sigma_{1} \dot{z}
$$

Given that $\dot{x}$ is very small in the stick-slip friction behavior, the term can be ignored. And the $\left(\frac{\dot{x}}{v 0}\right)^{2}$ in (5.10) is small. Thus, (5.10) is reduced to [Kang 2007; Canudas de Wit et al., 1995]

$$
g(\dot{x})=\lim _{\dot{x} \rightarrow 0} F_{C}+\left(F_{b}-F_{C}\right) e^{-\left(\frac{\dot{x}}{v 0}\right)^{2}}=F_{S S}
$$

where $F_{S S}$ is a constant having similar position of the $F_{C}$ in Dahl model. From the above, it is seen that $g(\dot{x})$ is simplified from 3-parameter function into single parameter. Therefore the (5.9) can be simplified to

$$
\frac{d_{z}}{d_{t}}=v-\sigma_{0} \frac{|\dot{x}|}{F_{S S}} Z
$$

LuGre model can be simplified to a 3-parameter model as follows

$$
\begin{gathered}
F_{f}=\sigma_{0} z+\sigma_{1} \dot{z} \\
\frac{d_{z}}{d_{t}}=\dot{x}-\sigma_{0} \frac{|\dot{x}|}{F_{S S}} Z
\end{gathered}
$$

This is the simplified LuGre model for the stick-slip application. 
It is noted that all of existing friction models including the aforementioned modified LuGre model were developed for the normal uniform isotropic friction surfaces, which have the same friction in two opposite directions. There is a need to represent the friction of the new AF surfaces, where the friction is dependent on the direction of their relative motion. Therefore, a modification on existing friction model is necessary. For this purpose, the method used in this study is to consider the parameters in the friction model depending on the motion direction. Specifically, in the simplified LuGre model outlined previously, there are three parameters, i.e., $\sigma_{0}, \sigma_{1}$ and $F_{S S}$, which are identified experimentally in a given application. To represent the anisotropic friction, these three parameters are considered as direction-dependent parameters. In other words, for each parameter, its value in one direction of the relative motion between endeffector and platform is different from that in the other direction A. Equation (5.22) shows the directional parameters in the friction model

$$
\sigma_{n}=\left\{\begin{array}{l}
\text { value } 1 \text { if } \dot{x} \geq 0 \\
\text { value } 2 \text { if } \dot{x}<0
\end{array}\right.
$$

For simulation in Simulink, Figure 5.1 shows the realization of the above equation where $\sigma_{n}$ can be any one of three parameters of $\sigma_{1}, \sigma_{2}, F_{S S}$, as well as $v$ is the velocity of relative motion, value 1 and 2 the values of the parameter in two opposite directions. 


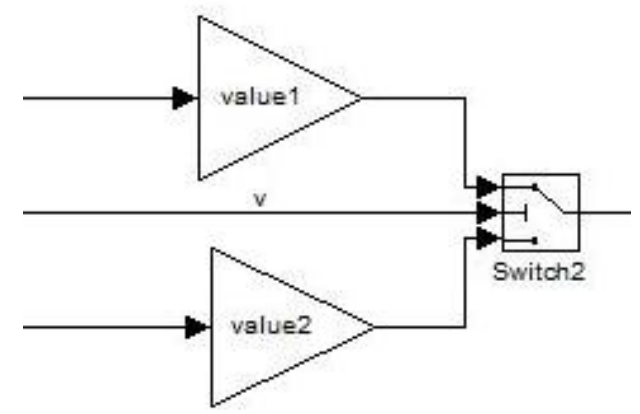

Figure 5.2 Realization of the direction-dependent parameter in Simulink.

Thus, each parameter in the previous simplified LuGre model has two values, depending on the relative motion between the end-effector and the platform. In the reminder of this chapter the three parameters, i.e., $\sigma_{0}, \sigma_{1}$, and $F_{S S}$, are replaced by six parameters which are denoted as $\sigma_{0 a}, \sigma_{0 b}, \sigma_{1 a}, \sigma_{1 b}, F_{S S a}$, and $F_{S S b}$ for two motion directions, respectively. Figure 5.2 shows the Simulink model developed for the friction of the IF surfaces based on the simplified LuGre friction model with three parameters It can be seen that there are three parameters circled by red ellipses, which are sigma_0, sigma_l and $F_{S S}$. The inputs to the model are the displacement of the end-effector (denoted by $x$-e) and the displacement of platform (denoted by $y$ ). This simplified LuGre friction model is referred as Isotropic friction model. Figure 5.3 shows the Simulink model for the friction of the AF surfaces based on the modified LuGre friction model with 6 parameters, which is referred as Anisotropic friction model. The difference between Figure 5.3 and Figure 5.2 is the three parameters in Figure 5.2 are replaced by the Simulink blocks representing the motion-related parameters illustrated in Figure 5.1. This modified sixparameter LuGre friction model has the capacity of representing the friction behaviour of the AF surfaces. 


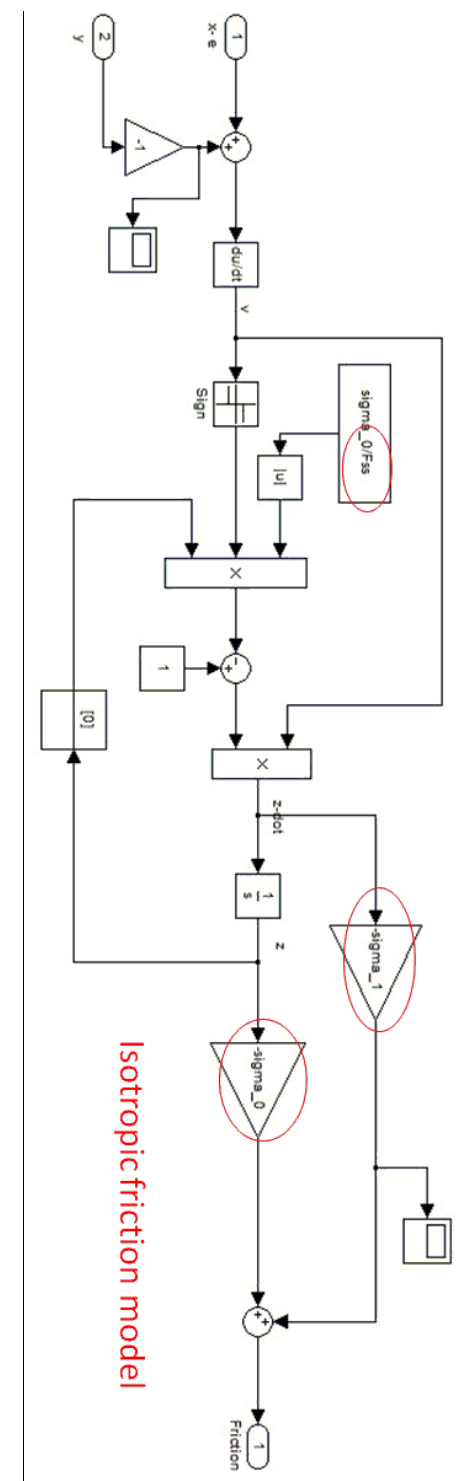

Figure 5.3 Simulink model based on the Simplified LuGre model with 3 parameters. 


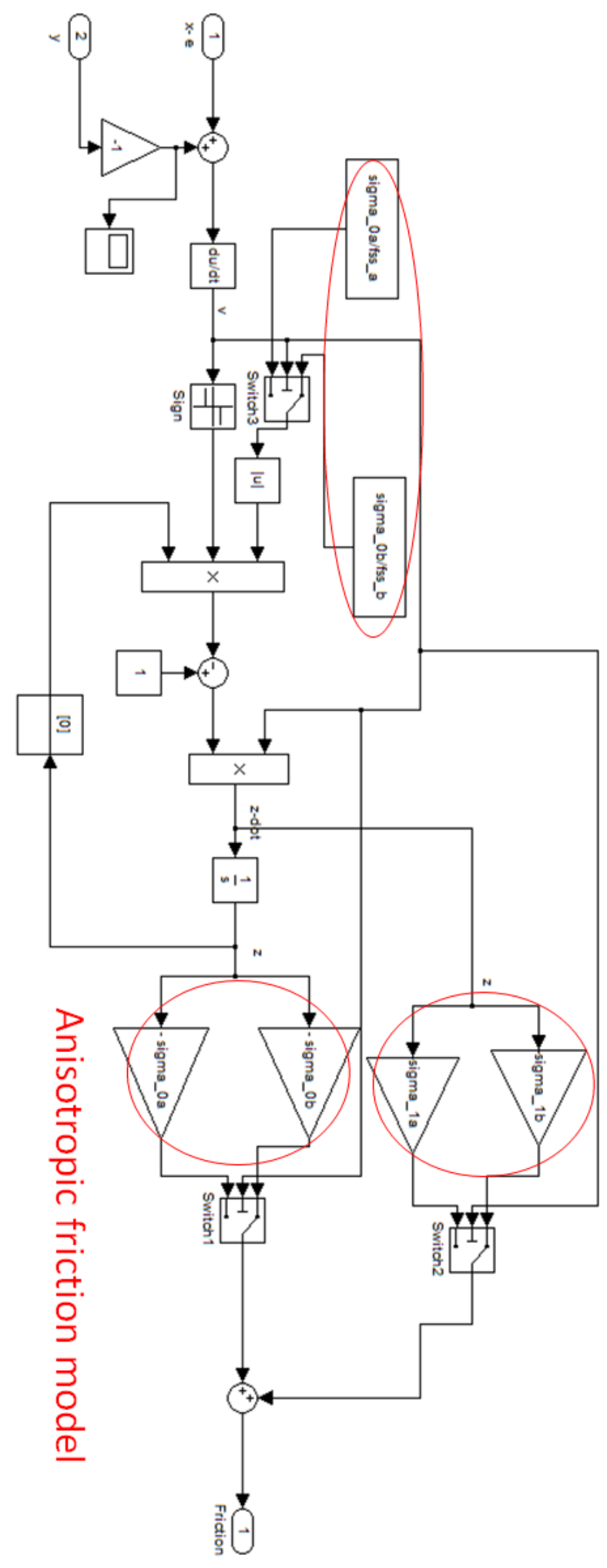

Figure 5.4 Simulink model based on the modified LuGre model with 6 parameters. 


\subsection{Modeling the PDSS Actuators}

From Chapter 4, it is known that a PEA is usually modeled by using a second order system. A LuGre friction model is adopted and modified as discussed in Section 5.2. Then, by combining the PEA model, friction model, and other dynamic factors affecting the motion of the end-effector together, a model for PDSS actuators can be developed, which is shown schematically in Figure 5.4.

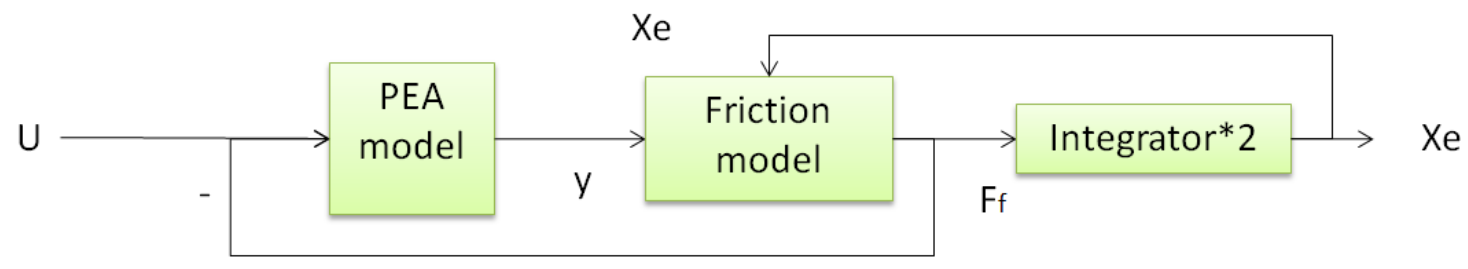

Figure 5.5 Schematic of the model for the PDSS actuator.

In Figure 5.4, $U$ is the applied voltage to the PEA, $y$ is the displacement of platform due to the contraction and elongation of the PEA, $X_{e}$ is the displacement of the end-effector, and $F_{f}$ is the friction force between the end-effector and the platform. Since the friction is the only force on end-effector by ignoring the damping force, the motion of end-effector is governed by

$$
M_{e} \ddot{x}_{e}=F_{f}
$$

It is noted from the previous discussion about friction model that $x$ denotes the relative motion between end-effector and platform, which is different between the displacement of end-effector $\left(x_{e}\right)$ and the displacement of platform $(y)$, i.e., 


$$
x=x_{e}-y
$$

For the PEA model in the Figure 5.4, it has the form of

$$
m_{g} \ddot{y}+c_{g} \dot{y}+k_{g}=T u-F_{f}
$$

For the friction model, it has the general form of

$$
F_{f}=\sigma_{0} z+\sigma_{1} \dot{z}
$$

By considering the mass of end-effector $(0.86 \mathrm{~g})$ and the output force of PEA (maximum value is $800 \mathrm{~N}$ [NEC/TOKIN]), it is noted that $F_{f}$ is smaller than $0.8 \mathrm{~N}$ (actually it is usually under $0.4 \mathrm{~N})$, which means the effect of $F_{f}$ for PEA's elongation is smaller than $0.1 \%(0.8 \mathrm{~N} / 800$ $\mathrm{N})$. Therefore, it is assumed that the feedback from the friction $F_{f}$ to the input signal $U$ is ignored. Under this assumption, the PDSS actuator model has a form

$$
m_{g} \ddot{y}+c_{g} \dot{y}+k_{g}=T u
$$

and it is schematically shown in Figure 5.5.

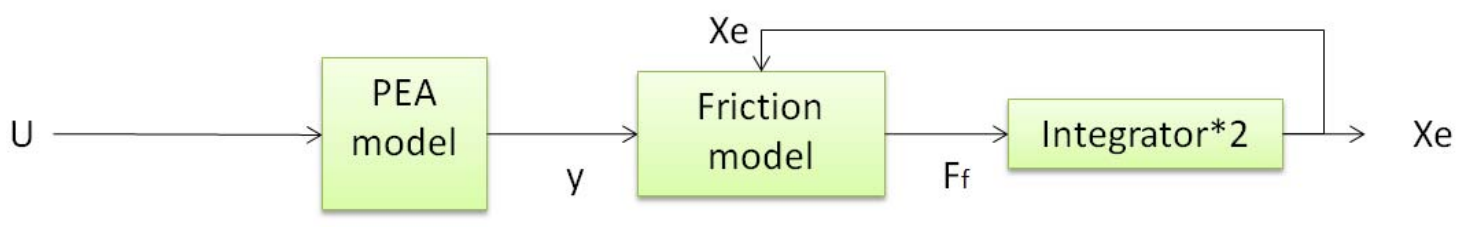

Figure 5.6 Simplified PDSS actuator model. 
As a second order system, Equation (5.27) can be expressed by transfer function in the form of

$$
\begin{gathered}
\frac{Y(s)}{U(s)}=G(s) \\
G(s)=\frac{C \omega^{2}}{s^{2}+2 \xi \omega s+\omega^{2}}
\end{gathered}
$$

where $Y(s)$ is the output signal in the $s$ domain, $U(s)$ the input signal in the $s$ domain, $G(s)$ the transfer function, $C$ the steady state gain, $\omega$ the natural frequency, $\xi$ the damping ratio. The parameters in Equation (5.29) were identified as $\omega=6736 \mathrm{rad} / \mathrm{s}, \xi=0.1772, C=1.007 \times 10^{-4}$ $\mathrm{m} / \mathrm{V}$ by the experiments and program in section 4.4 .

Although the nonlinearity of the PEA has drawn a lot of attention from researchers, in most applications the PEA was simply treated as a linear device [Pohl, 1987; Chang and Li, 1999]. In this study the nonlinearity of PEA such as hysteresis and creep is also ignored for this stick-slip application for the following reasons. Firstly, the elongation of PEA is far less than its full length elongation in the experiments conducted in this study. The PEA used has a full length of its elongation of $17.4 \pm 2.0 \mu m$ under 150 -volt driving voltage. In the experiments, it is not necessary to use the maximum elongation of the PEA under maximum voltage. Actually the maximum voltage applied to the PEA is less than 80 volts. In such a short elongation range, the effect of hysteresis appears less significant. Secondly, the dynamics of the combination of the PEA and the platform together shows the linear character by experiments. As discussed in Chapter 4, the PEA is not used separately, but used with a positioning mechanism; and if the 
mass of positioning mechanism is much larger than the mass of PEA, it is found in the experiments that the dynamics of the combination of the PEA and platform is dominated by a second order system.

\subsection{Experimental Verification}

\subsubsection{Parameter Identification}

To determine the parameters involved in the friction model, experiments were designed and conducted, which are detailed as follows.

1. All the experiments were conducted at the room temperature.

2. The displacement of the end-effecter under the sawtooth wave input was measured by using the Kaman inductive displacement sensor.

3. The end-effector with an aluminum plate attached can freely slide on the platform that was driven by the PEA.

For the data acquisition, the following settings were applied.

1. The magnitude of the sawtooth wave applied to the piezoelectric actuator was set to $64.2 \mathrm{~V}$ and the frequency was set to $5 \mathrm{~Hz}$ and $20 \mathrm{~Hz}$.

2. The sampling interval was set $1 \mathrm{~ms}$ for the input with a frequency of $5 \mathrm{~Hz}$, and $0.5 \mathrm{~ms}$ with a frequency of $20 \mathrm{~Hz}$.

3. The experiment was set lasting 5 seconds for the $5 \mathrm{~Hz}$ experiment and 2.5 seconds for the $20 \mathrm{~Hz}$ experiment.

4. The time delay of the experimental setup was identified as 0.2 second and then applied for the data acquisition, i.e. the data acquisition process starts 0.2 second after the application of the voltage to the PEA. 
A part of the data obtained from the performance characterization in the preceding chapater was used for estimating the parameters of the friction model. Figure 5.2 show the data for the parameter estimation, which are the measured displacements as the sawtooth voltage with a magnitude of 64 volts and a frequency of $5 \mathrm{~Hz}$ was applied to the actuator. The first 1.6 second part of this curve is the measured displacement of the end-effector in direction A; the second 1.6 second part of this curve in direction B. A Simulink model was developed to simulate the displacement of the end-effector for the given values of the model parameters, which is shown in Figure 5.7. This Simulink model allow for the simulations of the end-effector motion in two directions.

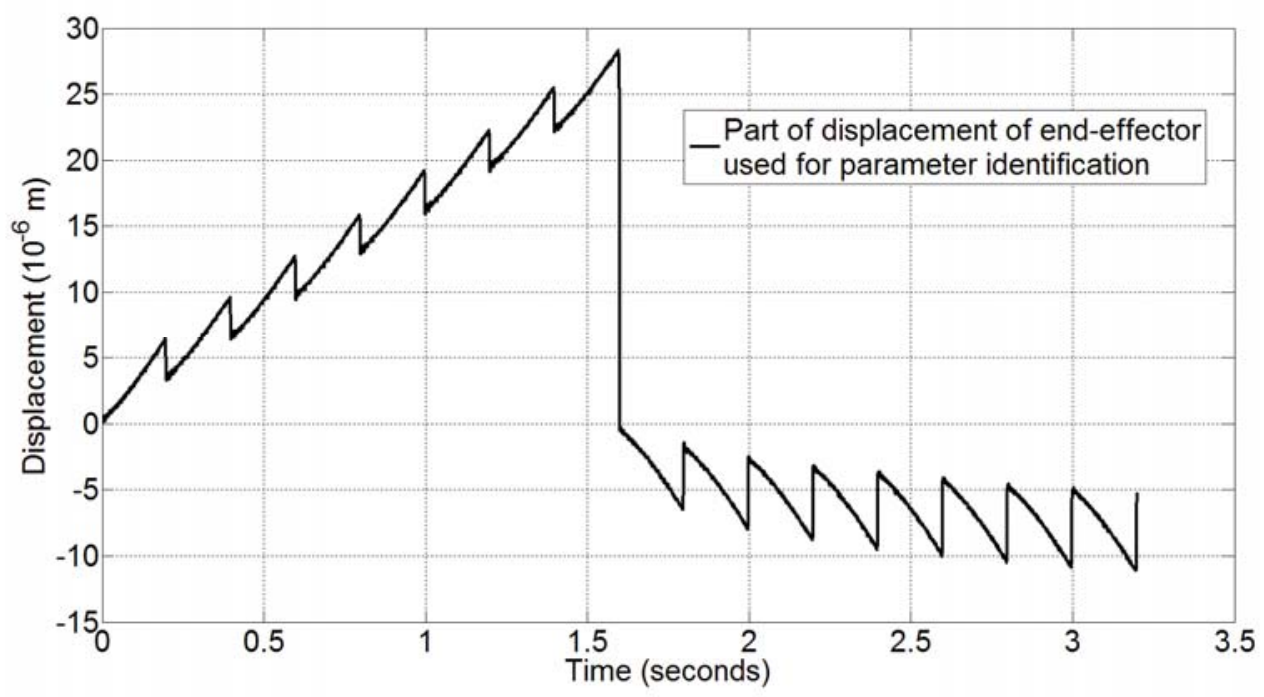

Figure 5.7 Experimental data for the parameter estimation. 


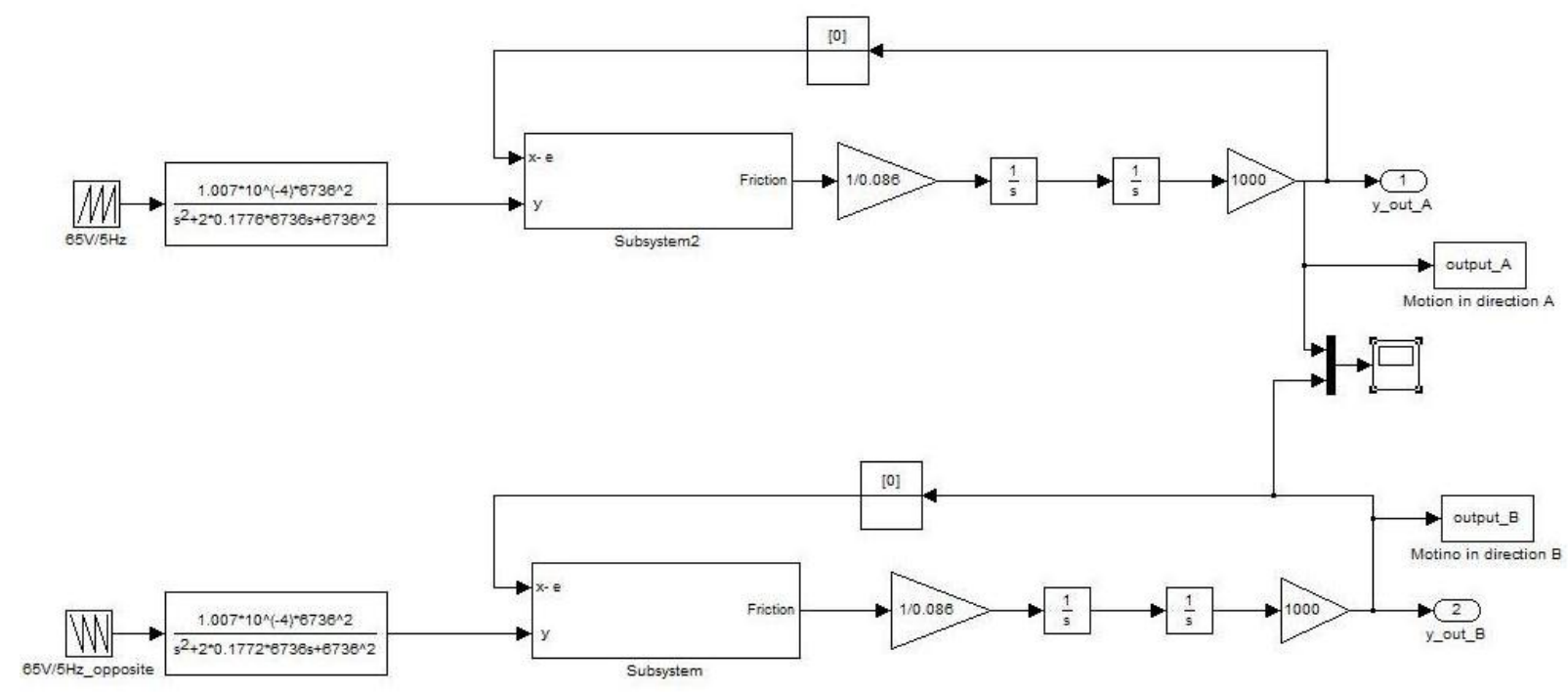

Figure 5.8 Simulink model used to simulate the motion of end-effector in two directions.

On basis of the above Simulink program of the novel PDSS actuator, the parameters of the friction model were estimated by employing the function of "nlinfit" in MATLAB. The function of "nlinfit" was employed to estimate the parameters, and the program codes are provided in Appendix 1. This function estimates the parameters by starting with an initial guess and then altering the guess until the error or the difference between the measured displacements and the model predictions is minimal. The MATLAB programs used to identify these six parameters are shown in Appendix 3. By doing so, the estimated values of the model parameters are $\sigma_{0 a}=1148.4 \mathrm{~N} / \mathrm{mm}, \sigma_{0 b}=953.789 \mathrm{~N} / \mathrm{mm}, \sigma_{1 a}=0.0544 \mathrm{Ns} / \mathrm{mm}, \sigma_{1 b}=0.0389 \mathrm{Ns} / \mathrm{mm}$, $F_{S S a}=0.7964 \mathrm{~N}$ and $F_{S S b}=0.5458 \mathrm{~N}$. With the estimated parameters, the simulated displacements by the developed model are also shown in Figure 5.2 in green for the comparison to the experimental data in black. 


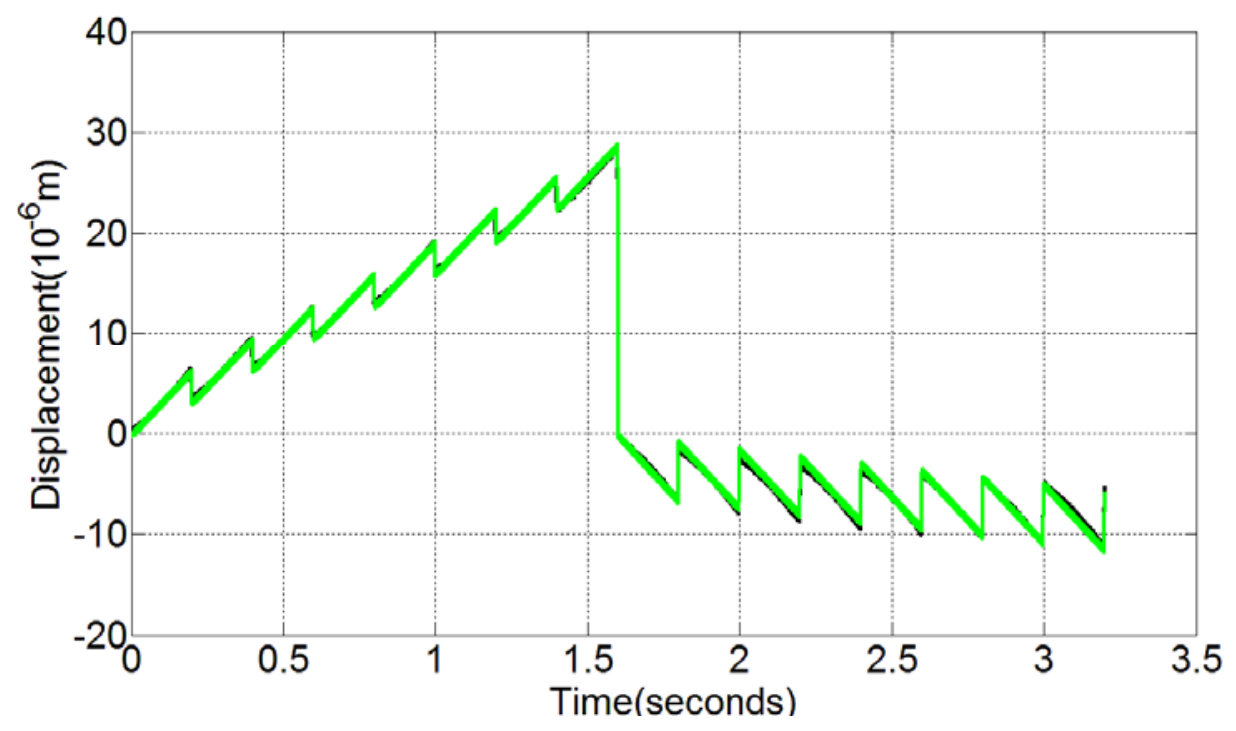

Figure 5.9 Comparison of the measured end-effector displacement and model predictions.

The parameters are used in the simulation and simulation result was compared to the experimental result. Figure 5.9 shows the comparison in 2 seconds working time. The simulation result agrees with the experimental result with the coefficient of determination $r^{2}$ of 0.9987 , which implies that the parameters were successfully identified.

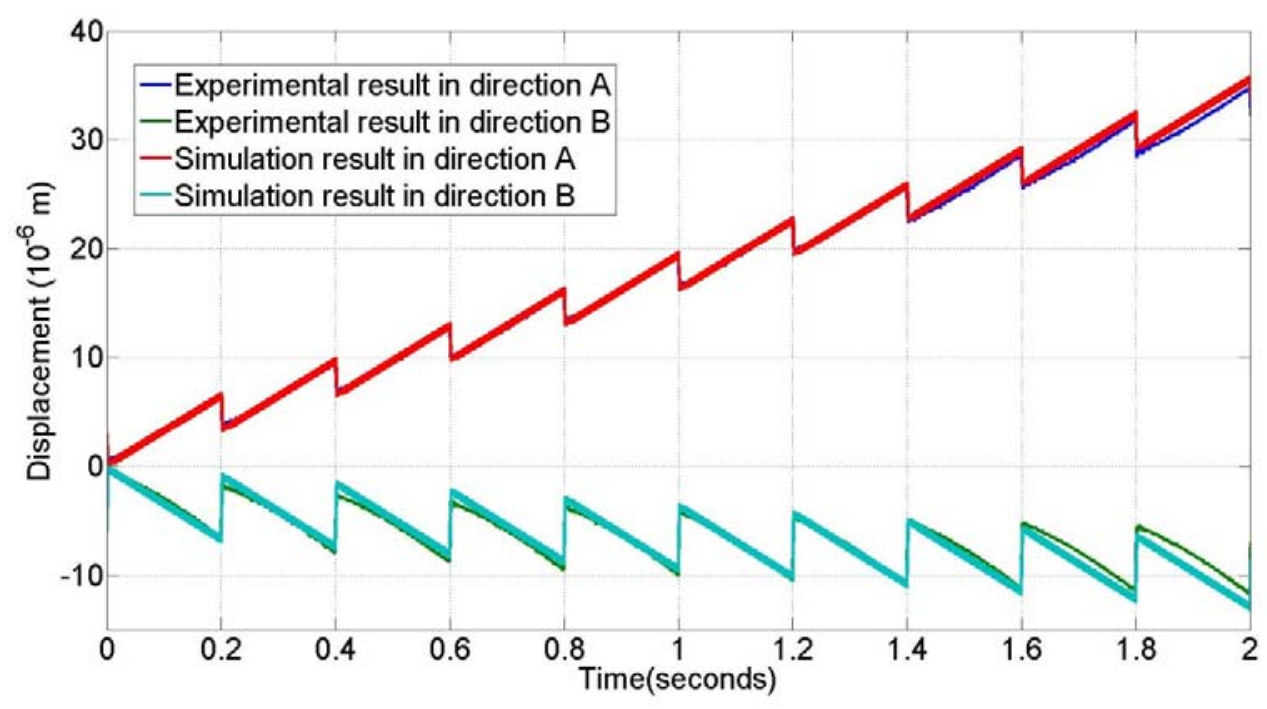

Figure 5.10 Experimental result matches simulation result. 


\subsubsection{PDSS Actuator Model Verification}

In order to verify the model, another experiment was conducted by applying $64 \mathrm{~V} / 20 \mathrm{~Hz}$ sawtooth wave to the PEA. The parameters identified previously were used in the Simulink model but the input was changed to the $64 \mathrm{~V} / 20 \mathrm{~Hz}$ for the simulation. The simulated displacement using $64 \mathrm{~V} / 20 \mathrm{~Hz}$ input was compared to the experimental displacement, which is shown in Figure 5.20. It can be seen that the simulation by applying the identified friction parameters agrees with the experimental results. The coefficient of determination $r^{2}$ was evaluated as 0.9946. A good agreement is seen between the measured displacements and model predictions, illustrating the promise of the developed model for the PDSS actuator with the AF surfaces.

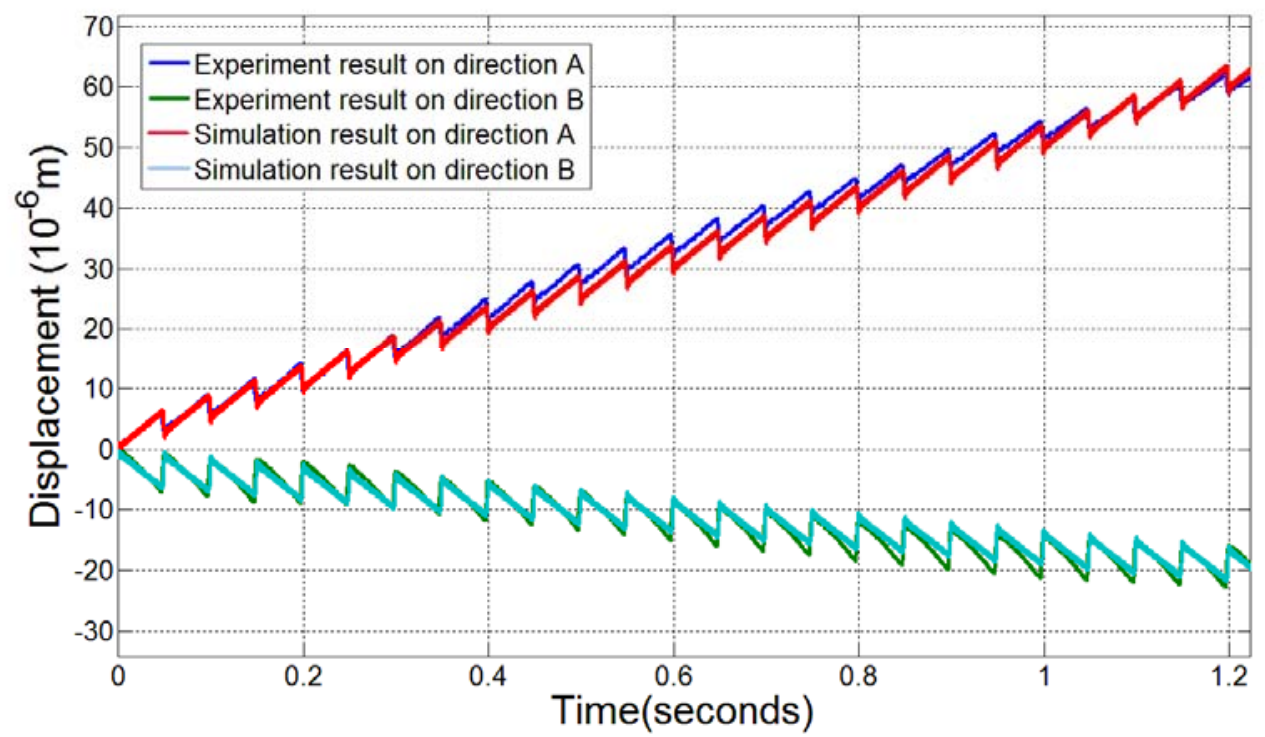

Figure 5.11 Verification of the novel PDSS actuator model through the comparison between experimental result and simulation result under $64 \mathrm{~V} / 20 \mathrm{~Hz}$ inputs. 


\subsection{Summary}

In this chapter, a model is developed to represent the friction of the AF surfaces and then the displacement of the end-effector driven by a PDSS actuator. First, several friction models are discussed and the LuGre model is adopted and modified from a 3-parameter model to a 6parameter model to represent the anisotropic friction behavior. Secondly, the PEA model, friction model and other dynamic factors of the end-effector are combined to obtain a PDSS actuator model. Thirdly, the parameters of this model are identified from the experimental results and the model is verified by comparing the simulation results to the experimental ones. The comparison indicates that the simulation results agree the experimental results; the model can predict the displacement of the end-effector. 


\section{CHAPTER 6 CONCLUSIONS AND FUTURE WORKS}

\subsection{Summaries and Conclusions}

Piezoelectric actuators (PEA) hold the most promise for the micropositioning applications due to their high precision. In many micropositioning and micromanipulation applications, the displacement range of an actuator is as vital as its precision. However, the small displacement range of the PEAs greatly restricts their applications. The piezoelectric-driven stick-slip (PDSS) actuator concerned in this research is one of the solutions to achieve long displacement by utilizing the stick-slip concept. Although the PDSS actuator has a capacity of achieving long displacement range, its performance leaves a lot to be desired. To improve their efficiency and loading capability, in this research the use of the working surfaces with anisotropic friction in the PDSS actuator was investigated, resulting in a novel PDSS actuator. The improvement in the efficiency and loading capability of the novel actuator was characterized by experiments. Also, a model representative of the improved performance of the PDSS actuators was developed.

The working surfaces named anisotropic-friction (AF) surface were constructed such that they could provide with different frictions dependent on the direction of their relative motion. 
The AF surfaces were created by applying the hot filament chemical vapor deposition (HFCVD) and ion beam etching (IBE) and the HFCVD was used to deposit diamond onto silicon substrates. After the deposition process, the IBE was used to etch the diamond coating with a selected angle to form asymmetric-triangle nanotips on the coating. On the other hand, another type of surface named isotropic-friction (IF) surface, which exhibits normal uniform friction behavior, was fabricated using the same technologies, but forming symmetric-triangle nanotips on the coating, rather than asymmetric-triangle nanotips on the AF surfaces.

A PDSS actuators was prototyped in this research and experiments were conducted on this prototype with AF surfaces and IF surfaces, respectively. The experimental results shows that, compared to the one using the conventional uniform IF surfaces, the PDSS actuator with AF surfaces has higher efficiency and higher loading capability, which suggests the use of the AF surfaces is a promising means to improve the performance of the PDSS actuator.

On the basis of the previous study on modeling of PDSS actuators, a model for the novel PDSS actuator with AF surfaces was developed to represent its improved performance. In the modeling process, the dynamics of the PEA and the platform was approximated by a second order dynamic system given the fact that the mass of platform was much larger than the mass of PEA. Another significant aspect of modeling, the presliding friction behavior, was represented by the simplified LuGre friction model. In order to represent the directional friction behavior of the AF surfaces, the simplified three-parameter LuGre friction model was modified by adding another three parameters. By combining the PEA mechanism model, friction model, and the dynamics of end-effector, a model for the PDSS actuator with the AF surface was developed. 
To verify the PDSS actuator model, the parameters of the actuator model were identified by the experiments conducted on the PDSS actuator prototype. The model with identified parameters was simulated via MATLAB Simulink and the simulation results obtained was compared to the experimental results. The comparison suggests that the model developed in this study is promising to represent the displacement of the novel PDSS actuators with AF surfaces.

The following conclusions are drawn in this research:

(1) The construction of the AF surfaces, which can provide direction-dependent friction, is achievable by employing the HFCVD and IBE techniques.

(2) The use of the AF surfaces is a promising means to improve the performance of PDSS actuators in terms of its efficiency and loading capability.

(3) The use of the second order approximation for the dynamics of the positioning system driven by a PEA is reasonable if the mass of the system is much greater than that of the PEA; and the method developed in this study to quantify the error associated with the approximation is effective.

(4) The simplified LuGre model with six parameters is able to represent the directiondependent friction of the AF surfaces. By combining the simplified LuGre model 
with the PEA dynamics model, the resultant model has the capability of representing the improved performance of the novel PDSS actuator.

\subsection{Future Works}

While the PDSS actuators developed in this study shows promising improvement compared to existing ones, there are still some issues needed to be addressed in the future research.

One piece of the future work could be the improvement of surface fabrication. One of the drawbacks of HFCVD is that the thickness of coating after deposition is not uniform, causing the coating surfaces uneven. Since the nanotips on the surfaces are in such a small size level compared to the unevenness of the surface caused by the deposition, the load concentrates in a small area and the nanotips in this small area are worn off easily because of its unevenness, especially when the end-effector is applied with a large blocking force. MWCVD can achieve much better uniform or flat surfaces, but the equipments for MWCVD involved in this research have the limitation on size of sample and deposition time. Therefore another better deposition technique is needed to achieve the coating in micrometer level or even in nanometer level, thereby avoiding the concentration of load in a small area and reducing the wear of AF surfaces.

Another piece of the future work could be the implementation of control schemes on the PDSS actuator for improved performance. The PDSS actuator prototype in this research is an open loop system. There should be a space from improving the performance of the PDSS actuator by introducing a controller in the system. 
The last piece of work suggested for the future is the improvement of parameter identification method for the friction model. In this research, the experimental data used in parameter identification for PEA model is the step response of PEA and the experimental data used in parameter identification for friction model is the response to the sawtooth wave form input. Here comes a question: how much error is brought into the friction model parameter identification from the PEA model parameter identification? These two steps were not independent, but successive. The error from the first step was accumulated to the second one by using the parameters identified in the first step. A few attempts were made to identify the parameters of friction model independently from the PEA model, thus reducing the propagation of the error of using PEA model to the friction model parameter identification. Although those attempts did not accomplish the desired improvement, they are worthwhile to pursue in the future. 


\section{REFERENCES}

[1] Adriaens, H.J.M.T.S., Koning, W. L. De and Banning, R. (2000) "Modeling Piezoelectric Actuators", IEEE/ASME Transactions on Mechatronics 5(4): 311-341.

[2] Bergander, A., Breguet, J.-M. and Clavel, R. (2003) "Micropositioners for microscopy applications and microbiology based on piezoelectric actuators", Journal of Micromechatronics 2(1): 65-76.

[3] Bliman, P. A. and Sorine, M. (1991) "Friction modelling by hysteresis operators", In Proceedings of the Conference Models of Hysteresis, Trento, Italy.

[4] Bliman, P. A. and Sorine, M. (1993) "A system-theoretic approach of systems with hsteresis", In Proceedings of Second European Control Conference, Groningen, The Netherlans, 1844-1849.

[5] Bliman, P. A. and Sorine, M. (1995) "Easy-to-use realistic dry friction models for automatic control", In Proceedings of $3^{\text {rd }}$ European Control conference, Rome, Italy, pp: $3788-3794$.

[6] Burleigh Instruments, (1998) “The Technology behind Burleigh Inchworm Systems”, Burleigh Instruments, Fishers, NY.

[7] Canudas de Wit, C., Ollson, H., Astrom, K J. and Lischinsky, P. (1995) “A new model for control of system with friction", IEEE Transactions on Automatic Control 40(3): 419-25.

[8] Chang, S. H. and Li, S. S. (1999) "A high resolution long travel friction-drive micropositioner with programmable step size", Review of Scientific Instruments 70: 2276-2282. 
[9] Chen, Q., Yao, Da-J., Chang-Jin 'CJ' Kim and Greg P. Carman (1999) "Mesoscale actuator device: micro interlocking mechanism to transfer macro load", Sensors and Actuators A: Physical 73(1-2): 30-36.

[10] Chen, D., Kang, D. and Zhang, Q.S. (2008) "On the Dynamics of PiezoelectricDriven Stick-Slip Actuators”, Key Engineering Materials, 375: 648-652.

[11] Dahl, P. (1968) “A solid friction model”, Technical Report Tor-0158(3107-18)-1, The Aerospace Corporation, EI Segundo, CA.

[12] Dahl, P. (1975) "Solid friction damping of spacecraft oscillations", AIAA Paper No. 75-1104 presented at the AIAA Guidance and Control Conference, Boston Mass.

[13] Darby, A. P. and Pellegrino, S. (1999) "Modeling and control of a flexible structure incorporating inertial slip-stick actuators", Journal of Guidance, Control, and Dynamics 22(1): 36-42.

[14] Dupont, P., Hayward, V., Armstrong, B. and Altpeter, F. (2002) "Single State Elastoplastic Friction Models", IEEE Transactions on Automatic Control 47(5): 787792.

[15] Dupont, P., Armstrong, B. and Hayward, V. (2000) "Elasto-plastic Friction Model: Contact Compliance and Stiction", American Control Conference, Proceedings of the 2000 2: 1072-1077.

[16] Leonard, N. E. and Krishnaprasas, P. (1992) “Adaptive friction compensation for bidirectional low-velocity position tracking", In Proc. Of the $31^{\text {st }}$ Conference on Decision and Control, pages 267-273.

[17] Ferretti, G., Magnani, G. and Rocco, P. (2004) "Single and multistate integral friction models", IEEE Transactions on Automatic Control 49: 2292-2297. 
[18] Goldfarb, M. and Celanovic, N. (1997) "Modeling piezoelectric stack actuators for control of micromanipulation", IEEE Control Systems Magazine 17: 69-79.

[19] Gomes, S.C.P., Santos da Rosa, V. (2003) "A new approach to compensate friction inrobotic actuators", Robotics and Automation, Proceedings. ICRA 03. IEEE International Conference on 1: 622-627.

[20] Haessig, D. A., Friedland, B. (1991) “On the Modeling and Simulation of Friction", Journal of Dynamic Systems, Measurement, and Control 113(3): 354-362.

[21] Hoogeman, M.S. et al., (1998) "Design and performance of a programmabletemperature scanning tunnelling microscope", Review of Scientific Instruments 69: 2072-2080.

[22] Kaman Instrumentation Corporation, "Application guidelines for inductive displacement measuring systems".

[23] Kaman Instrumentation Corporation, "SMU-9000 User Manual”.

[24] Kang, D. (2007) "Modeling of the piezoelectric-driven stick-slip actuators", Master thesis, Department of Mechanical Engineering, University of Saskatchewan.

[25] Liu, Y. T. and Higuchi, T. (2001) "Precision positioning device utilizing impact force of combined piezo-pneumatic actuator", IEEE Transactions on Mechatronics 6: 467473.

[26] NEC/TOKIN, "Multilayer Piezoelectric Actuators Vol. 02".

[27] Niezrecki, C., Brei, D., Balakrishnan, S. and Moskalik, A. (2001) "Piezoelectric actuation: state of the art", The Shock and Vibration Digest 33(4): 269-280.

[28] Norgate, P. and Hammond, V. J. (1974) "Ion Beam Etching”, Physics in Technology 5(3):186 -203. 
[29] Olsson, H. (1996) "Control Systems with Friction", PhD thesis, Lund Institute of Technology, University of Lund.

[30] Olsson, H., Astrom, K. J., Canudas, C., Wit, de and ets. (1998) "Friction models and friction compensation”, European Journal of Control 4: 176-195.

[31] Paj Bliman, "Mathematical study of the Dahl's friction model" European Journal of Mechanics. A. Solids 11:66, 835-848, Elsevier, 1992

[32] Palmer, J.A., Dessent, B., Mulling, J.F., Usher, T., Grant, E., Eischen, J.W., Kingon, A.I. and Franzon, P.D. (2004) "The design and characterization of a novel piezoelectric transducer-based linear motor", IEEE/ASME Transactions on Mechatronics 9(2): $392-398$.

[33] Park, S. E., and Shrout, T. R. (1997) "Ultrahigh Strain and Piezoelectric Behavior in Relaxor Based Ferroelectric Single Crystals," Journal of Applied Physics 82: 18041811.

[34] Philip, R. and Dahl, P. (1976) "Solid friction damping of mechanical vibrations", AIAA Journal, 14(12); 1675-82.

[35] Pohl, D.W. (1987) "Dynamic piezoelectric translation devices", Review of Scientific Instruments 58(1) January.

[36] Pozzi, M. and King, T. (2001) "Piezoelectric actuators in micropositioning", Engineering Science and Education Journal 10(1): 31-36.

[37] Renner, Ch., Niedermann, Ph., Kent, A.D. and Fischer (1990) "A vertical piezoelectric inertial slider", Review of Scientific Instruments 61: 965-967. 
[38] Salisbury, S.P., Waechter, D.F and etc. (2006) "Design considerations for complementary inchworm actuators”, Mechatronics, IEEE/ASME Transactions on 11(3): $265-272$.

[39] Sezer, Ali O. and Brand, J.I. (2001) "Chemical vapor deposition of boron carbide", Materials Science \& Engineering. B, Solid-State Materials for Advanced technology 79(3):191 -202.

[40] Swevers, J., Al-Bender, F., Ganseman, C.G. and Projogo, T. (2000) “An integrated friction model structure with improved presliding behavior for accurate friction compensation", IEEE Transactions on Automatic Control 45(4): 675 - 686.

[41] Rabinowics, E. (1951) "The nature of the static and kinetic coefficients of friction", Journal of Applied Physics 22(11).

[42] Tenzer, P. E. and Mrad, R. B. (2004) "A systematic procedure for the design of piezoelectric inchworm precision positioners", IEEE Transactions on Mechatronics 9: 427-435.

[43] Walrath, C. (1984) "Adaptive bearing friction compensation based on recent knowledge of dynamic friction", Automatica, 20(6): 717-727.

[44] Wu, Ruh-Hua and Tung, Pi-Cheng (2002) "Studies of stick-slip friction, presliding displacement, and hunting”, Journal of Dynamic Systems, Measurement, and Control 124(1): $111-117$

[45] Zhang, Y., Liu, G. and Hesselbach, J. (2006) "On development of a rotary-linear actuator using piezoelectric translators", IEEE Transactions on Mechatronics 11: 647-650. 
[46] Zhang, Y., Zhang, W.J., Hesselbach, J. and Kerle, H. (2006) "Development of a twodegree-of-freedom piezoelectric rotary-linear actuator with high driving force and unlimited linear movement”, Review of Scientific Instruments 77:035112. 


\section{APPENDIX}

\section{Appendix 1 MATLAB Program Used to Identify the Parameters of the Second Order System.}

\section{Program A1.1}

$\%$ file name pro_parameter_iden_sos.

$\%$ This program is used to identify the three parameters of the second order \% system. three step response under 48/64/75V voltages input are used for $\%$ analysis. This file focuses on preparing experiment data for the further $\%$ analysis in the function "para_ide": the main function to identify the $\%$ parameter.

$\%$ the final result is the natural frequency $W$; damping Q; gain KO clc, clear, clf;

tFinal $=0.035$

p०1=10;

\%1. load three groups of experiment data

load 20081_5mass_482v2

$\mathrm{x}=$ trace_x;

$y(1,:)=$ trace $\_y(1,:)$;

load 20081_5mass_642v2

$\mathrm{y}(2,:)=$ trace_y $(1,:)$;

load 20081_5mass_753v2

$\mathrm{y}(3,:)=$ trace_y $(1,:)$;

\% start the analysis separatly for three step response

for $i=1: 3$

$\%$ 2. get the bias and cut the data with bias

$\operatorname{dias}(i)=\operatorname{mean}(y(i, 10: 90))$;

$y(i,:)=y(i,:)-\operatorname{dias}(i)$;

$y(i,:)=y(i,:) * 0.000001$;

$Y(i,:)=y(i, 102: 102+75)$;

$X=x(101: 101+75)$;

out $=Y(i,:) ; t=X$;

$\operatorname{plot}\left(X, Y(i,:) * 10 \wedge 6,{ }^{*}-^{\prime}\right)$ \%plot the prepared data

grid on

hold on

\% 3.get the three parameters

[Q W K] =para_ide $(\mathrm{Y}(\mathrm{i},:))$ \%used the function to get the three parameters $\mathrm{Q} \odot(\mathrm{i})=\mathrm{Q} ; \mathrm{W} \odot(\mathrm{i})=\mathrm{W} ; \mathrm{K} \odot(\mathrm{i})=\mathrm{K}$;

$\mathrm{G}=\mathrm{tf}\left(\mathrm{K}^{*} \mathrm{~W}^{\wedge} 2, \quad\left[1 \mathrm{C}^{*} \mathrm{~W}^{*} \mathrm{Q} \mathrm{W}^{\wedge} 2\right]\right) ;$

$T=[0: 0.00005: 0.035]$;

$\operatorname{res}(:, i)=\operatorname{step}(G, T)$;

$\operatorname{plot}\left(T, \operatorname{res}(:, i)^{\prime *} 10^{\wedge} 6,{ }^{\prime} \mathrm{k}--^{\prime}\right)$

hold on

$\%$ 4.calculate the errors

err $2=0$;

for $j=1: 60$

$\operatorname{err} 2=\operatorname{err} 2+(Y(i, j)-\operatorname{res}(j, i))^{\wedge} 2$;

end 


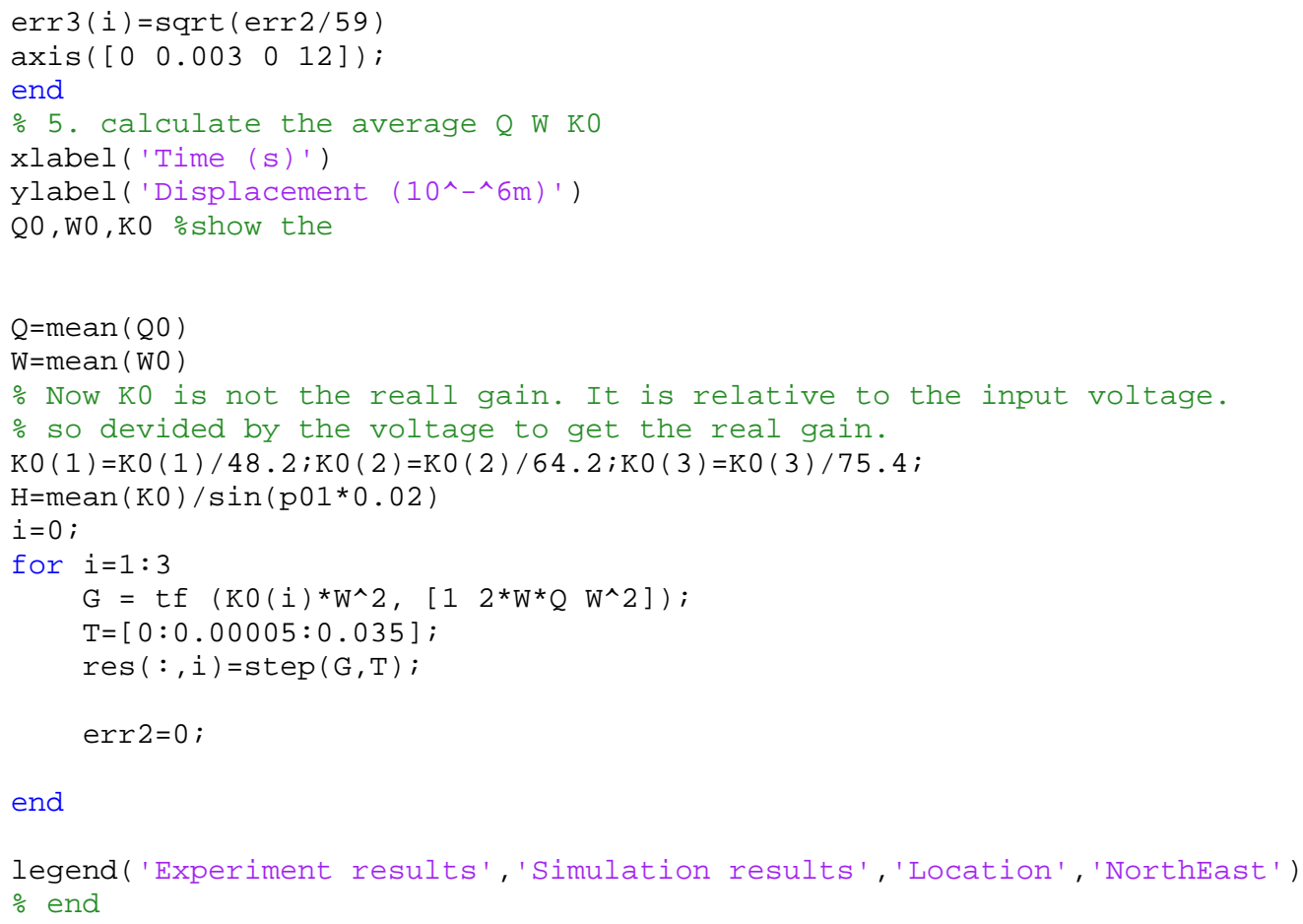

\section{Program A1.2}

$\%$ the function is used to identify the parameters of the second order $\%$ system of the PEA. The basic principle is to use 6 points( 3 top, 3buttom $\%$ points) of the step response to calculate the average $\mathrm{Tp}$, and $\mathrm{Ci}$ based \%on parameter $\mathrm{K}$. then using the regression to get a better $\mathrm{K}$ and update the \% c to get a better $\mathrm{C}$ to calculate the natural frequency and damping ratio.

\section{function $[\mathrm{Q}$ W K] $=$ para_ide(Yout)}

Y=Yout ;

\%---find the 6 peaks time.

$\mathrm{n}=\left[\begin{array}{llllll}1 & 2 & 3 & 4 & 5 & 6\end{array}\right]$; \% define the sequence

$[\mathrm{C}(1) \mathrm{I}(1)]=\max (\mathrm{Y})$; \% find the first Tp which is Tp1 peri=1. $7^{*} I(1) \quad \%$ pick up the period to find the next $\mathrm{Tp}$ $[C(2) I(2)]=\min (Y(I(1): \operatorname{peri}+\mathrm{I}(1)))$; \% find the next Tp $\mathrm{I}(2)=\mathrm{I}(2)+\mathrm{I}(1) ; \quad$ \%update the starting point for new period $[\mathrm{C}(3) \mathrm{I}(3)]=\max (\mathrm{Y}(\mathrm{I}(2): \operatorname{peri+I}(2)))$;

$\mathrm{I}(3)=\mathrm{I}(3)+\mathrm{I}(2)$;

$[\mathrm{C}(4) \mathrm{I}(4)]=\min (\mathrm{Y}(\mathrm{I}(3): \operatorname{peri}+\mathrm{I}(3)))$;

$\mathrm{I}(4)=\mathrm{I}(4)+\mathrm{I}(3)$;

$[C(5) I(5)]=\max (Y(I(4): \operatorname{peri}+\mathrm{I}(4)))$;

$\mathrm{I}(5)=\mathrm{I}(5)+\mathrm{I}(4)$;

$[C(6) I(6)]=\min (Y(I(5): \operatorname{peri}+\mathrm{I}(5)))$;

$\mathrm{I}(6)=\mathrm{I}(6)+\mathrm{I}(5)$;

$C ; I(1)=I(1)+1$;

$I=(I-2) * 0.000 \odot 5 ; \%$ get the real time instead of the point position

$\mathrm{Tp}=\operatorname{mean}(\mathrm{I} . / \mathrm{n}) ; \%$ get the average value of the $\mathrm{Tp}$

$\%$--decide $\mathrm{K}$ roughly and find the first a and start the regression $\mathrm{K}=\operatorname{mean}(\mathrm{C})$; 


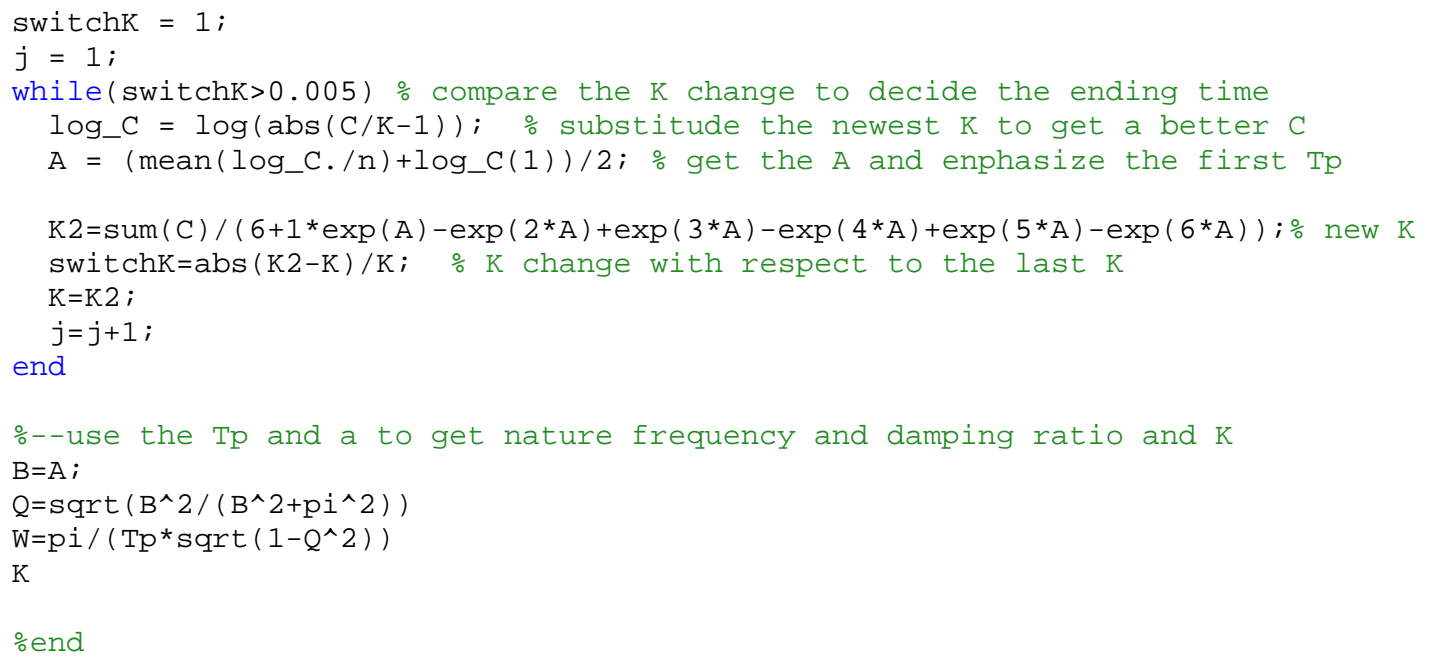

\%end

\section{Appendix 2 MATLAB Program Used to Draw Figure 4.7}

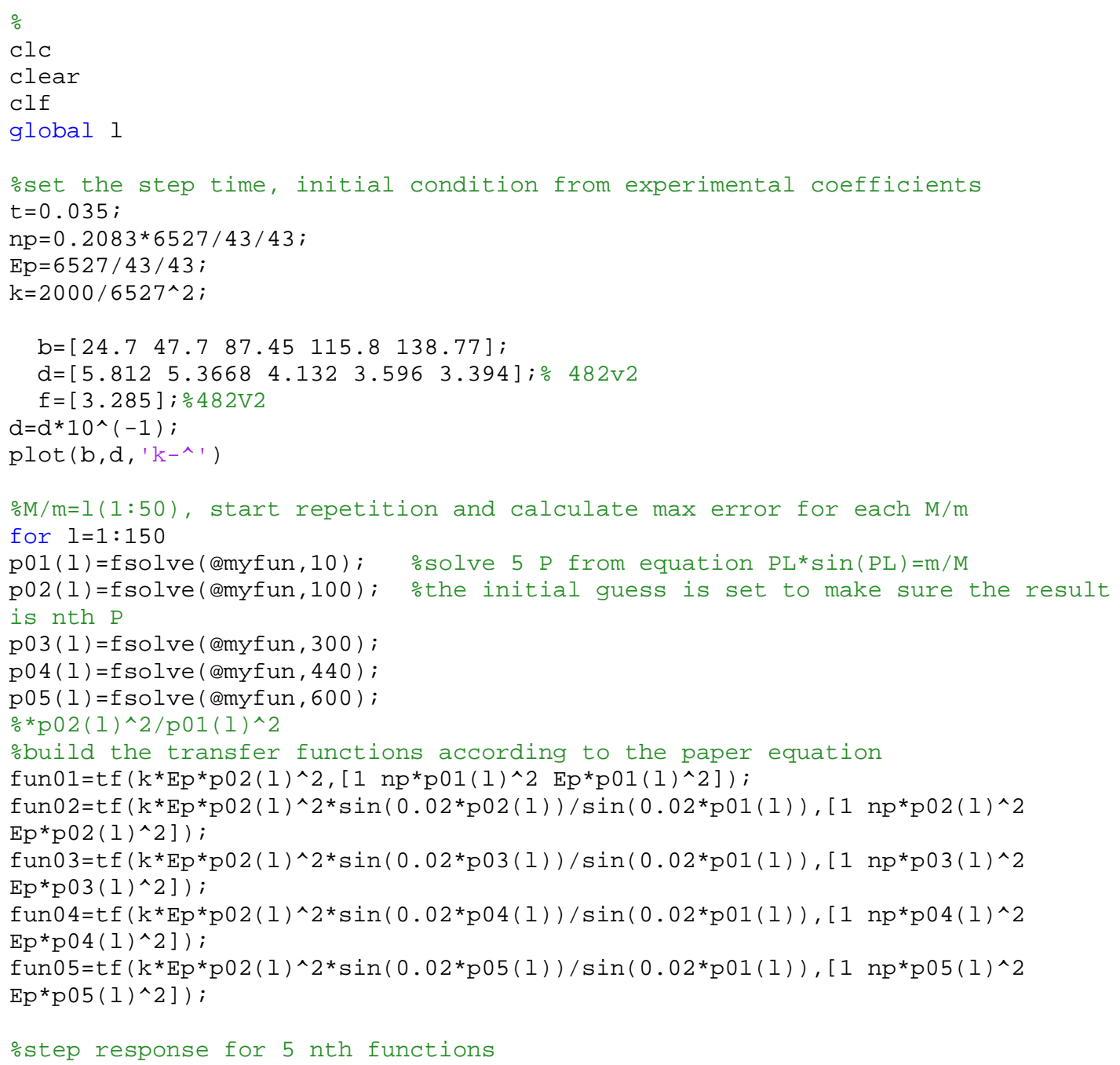




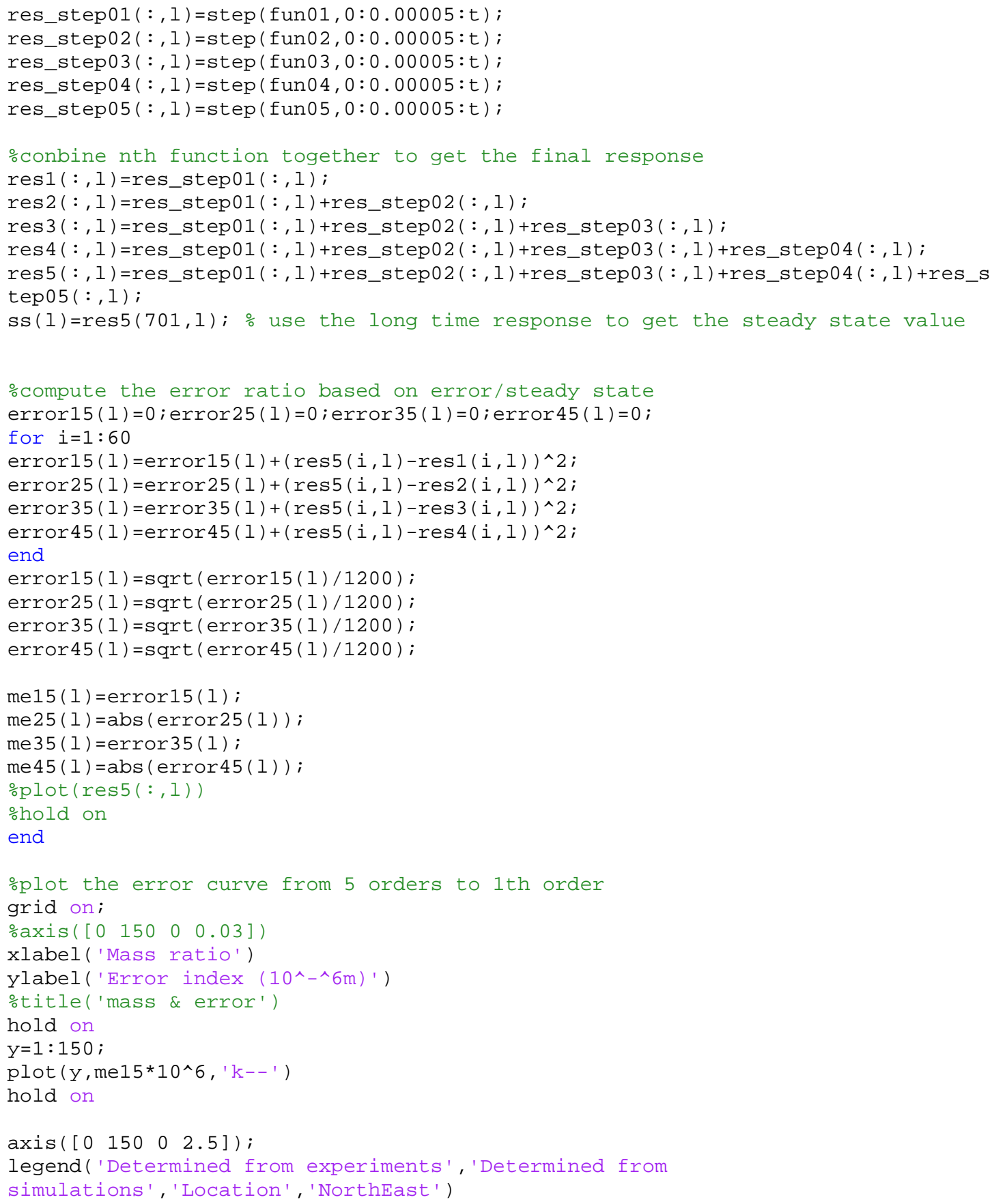

\section{Appendix 3 MATLAB Program Used to Identify the Parameters in Friction Model}

\section{Program A3.1}

\% Filename: nlinfit_PZT_Friction_damper_6var.m

\% Instruction: This program is for employing nlinfit in sawtooth experiment $\%$ data regression by using 3 variables. The data for

$\% \quad$ regression is cut from the initial extracted data and total 
$\%$

8 waves. And calculate the coefficient of determination.

$\%$ function [sigma_0a, sigma_ob,sigma_1a,sigma_1b,fss_a,fss_b] =

nlinfit_PZT_Friction_damper_6var(volt)

clc, clear, clf;

global volt;

global voltage; voltage=64.2;

global Ped_s;

global sigma_oa;

global sigma_ob;

global sigma_1a;

global sigma_1b;

global fss_a;

global fss_b;

global freq;

global pn s;

global Dis_cut;

$\% 5 \mathrm{~Hz}$ sawtooth regression with nlinfit

\% (1) Choose different frequency start simulation,

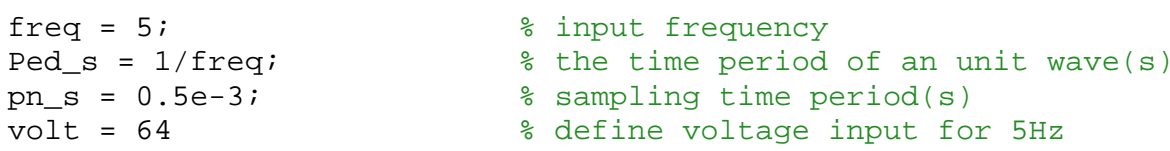

\% (2) Run Experiment_results_extraction_5Hz.m

$\%$ and load the results.

load Feat_65v5hz3

t_cut=X; Dis_cut=F ;

my_opts = statset ('Derivstep',6.000e-008)

$\%$ (3) Regression of $5 \mathrm{~Hz}$ data with nlinfit to determine parameters

$\%$ make initial values of model

sigma_in $=[1148.4,953.789,0.0544,0.0389,0.7964,0.5458]$;

[sigma_out, $r, \mathrm{~J}]=$

nlinfit( t_cut,Dis_cut,@nlffuc_6var_single_qsz,sigma_in,my_opts );

\% (4) plotting the regression figure

[yFit, delta] = nlpredci('nlffuc_6var_single_qsz',t_cut,sigma_out,r, J);

plot(t_cut,Dis_cut, 'k- ',t_cut,yFit, 'g-' ), grid on;

hold on;

rsqu $=\operatorname{cod}($ Dis_cut, yFit) $\quad \%$ calculate the coefficient of

$\%$ determination

time_1 = t_cut;

Displa_1 = Dis_cut; 


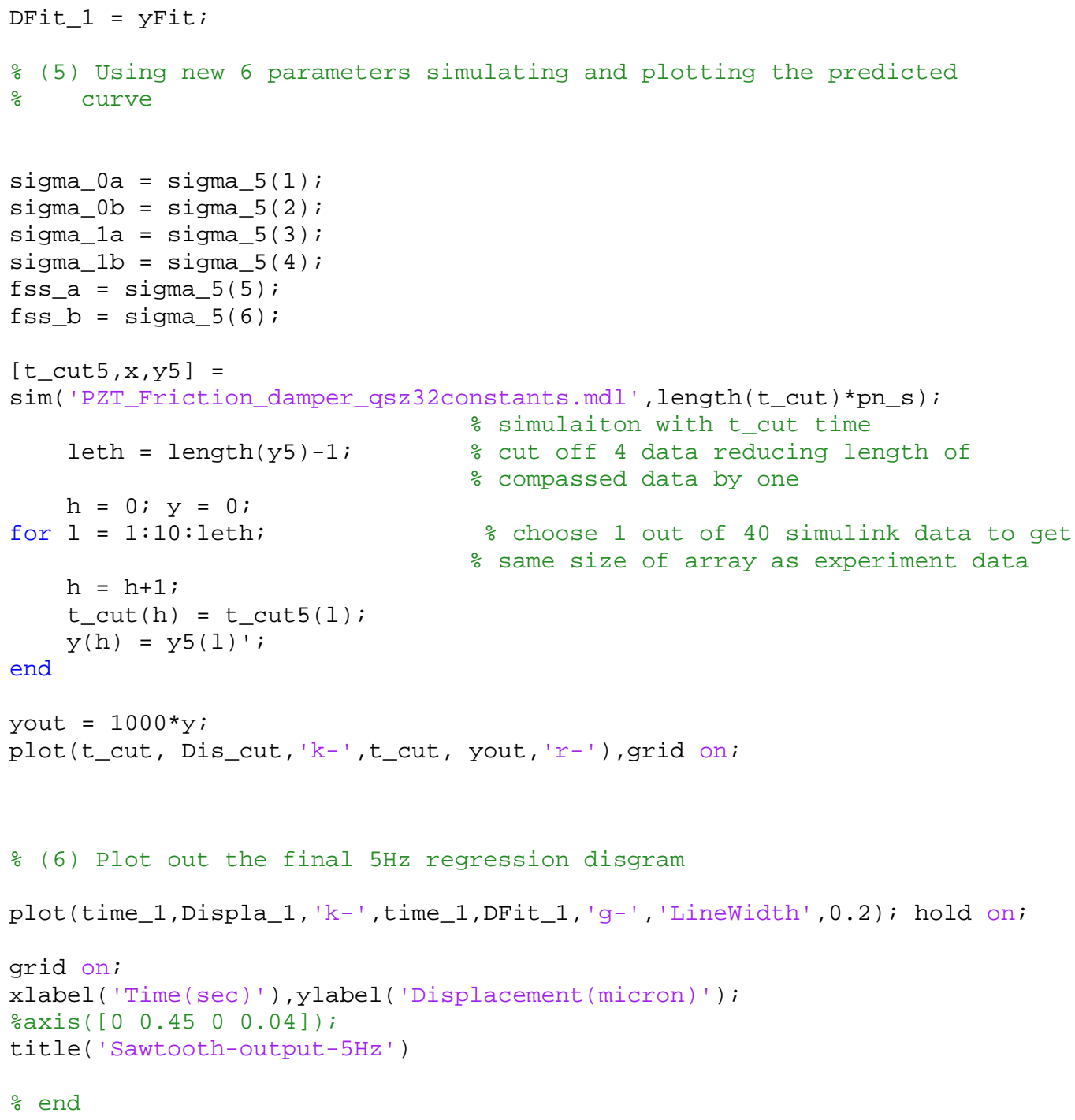

\% (6) Plot out the final $5 \mathrm{~Hz}$ regression disgram

plot(time_1,Displa_1, 'k-',time_1,DFit_1,'g-' ,'LineWidth',0.2); hold on;

grid on;

xlabel('Time(sec)'), ylabel('Displacement(micron)' );

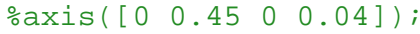

title( 'Sawtooth-output-5Hz')

$\%$ end

\section{Program A3.2}

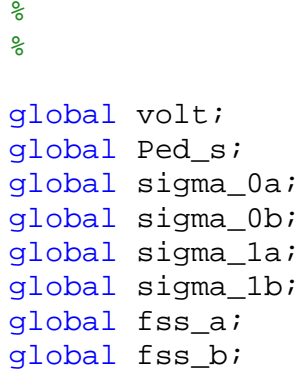




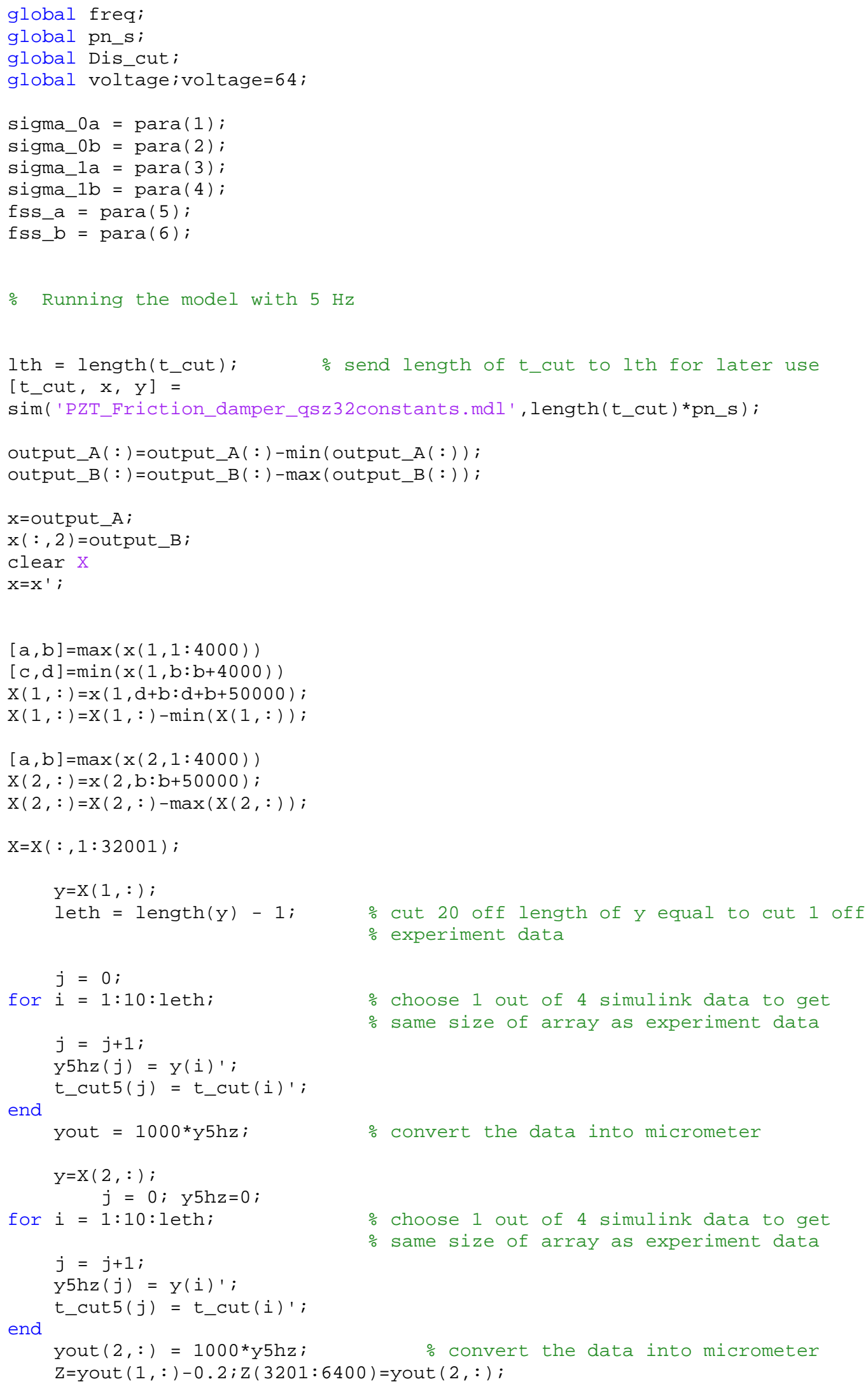

$\%$ choose 1 out of 4 simulink data to get $\%$ same size of array as experiment data 
t_cut $=\left[t \_c u t 5, t \_c u t 5(3200)+t \_c u t 5\right] ;$

sigma_0a

sigma_ob

sigma_1a

sigma_1b

fss_a

fss_b

plot(t_cut, z, 'b- ',t_cut, Dis_cut, 'k-' );

yout=Z;

$\%$ The end 